\title{
Axiomatic Affine Unification with Large Gravitational Vector Field Yields Vector-Metric Theory of Gravitation, Electromagnetism and Field Description of Mass-Particles
}

\author{
Boris Hikin \\ Artwork Conversion, Los Angeles, USA \\ Email: blhikin@gmail.com
}

How to cite this paper: Hikin, B. (2017) Axiomatic Affine Unification with Large Gravitational Vector Field Yields Vector-Metric Theory of Gravitation, Electromagnetism and Field Description of Mass-Particles. Journal of High Energy Physics, Gravitation and Cosmology, 3, 178-247.

https://doi.org/10.4236/jhepgc.2017.32019

Received: December 27, 2016

Accepted: March 13, 2017

Published: March 16, 2017

Copyright $\odot 2017$ by author and Scientific Research Publishing Inc. This work is licensed under the Creative Commons Attribution International License (CC BY 4.0).

http://creativecommons.org/licenses/by/4.0/

\begin{abstract}
Under assumption of existence of extremely large gravitational vector field, this paper proposes a road map for building an Axiomatic Eddington Affine Unification theory yielding both Maxwell's electromagnetism and Vectormetric theory of gravitation, in which inverse of the square-magnitude of the vector serves as Newton's gravitational constant. The dependence of the vector's magnitude with distance may offer an explanation of both Pioneer anomaly and "star rotation abnormality" in some Galaxies. In addition, the theory provides formalism for a classical description of atomic particles (such as protons and electrons) with highly non-linear equations and highly localized solutions. The existence of large Gravitational vector field can, for some variables (sub-fields), lead to elliptical type $\left(\partial_{t t}+\partial_{x x}\right)$ differential equations (unlike in Maxwell's electromagnetism, which is hyperbolic $\partial_{t t}-\partial_{x x}$ ), that by its nature forbids the existence independent waves and their propagation. Proposed Unified field description might provide the avenue for smooth transition to the world Quantum physics.
\end{abstract}

\section{Keywords}

General Relativity, Affine Unification, Vector-Metric Gravitation, Gravitational Constant, Emerging Gravitation, Large Gravitational Field, Dark Matter

\section{Short Summary of the Paper and Its Results}

This paper presents a road map for building a unified field description of matter. It describes the physical basis (postulates) and the physical phenomenon in more or less generic terms and writes most formula in symbolic manner. But even in 
such general description we demonstrate that this theory produces comparable (if not superior) formulation of Gravitation and Electromagnetism as well as 2 other vector fields that act similarly to neutrino and Bozon particles. In addition it does provide avenue for building field theory of mass-particles, such as electrons and protons.

Our starting point is our desire to derive Newton gravitational constant, and in fact the entire gravitation, thru only atomic "interaction" (i.e. some property that are present in every atomic formation) and atomic units coupled with the number of particles in the Universe (or at least of our Galaxy).

We use Affine description only as an avenue for obtaining the Lagrangian and the covariant set of equations that describe the evolution of the fields. We do not subscribe to any geometrical interpretation and/or any "geometrization" of physical forces or physical entities other than metric tensor that describes the curved space.

For the majority of physicists who undoubtedly reject the field unification idea strictly on the grounds that true description of matter must be Quantum Mechanical, may I suggest this thought for consideration? One can view field description proposed here as a classical limit of quantum mechanical wave functions description and use it as a starting point for quantization as it has been, for example, done for bozon quantum field theory, which starts as a classical nonzero mass vector field.

1) Our main assumption is such that gravitation is described as two entities: metric tensor $\mathbf{r} \equiv r_{i j}$ and by a Gravitational vector $\mathbf{G} \equiv G_{i}$ which is an attribute of all particles (or at least the heavy ones) just like Electromagnetic field is an attribute of all charged particle. The difference though is that the Gravitational field always comes with "the same sign" sort of speak and thus is allowed to accumulate and reaches in our Milky Way Galaxy an enormous value of $10^{33} \frac{1}{\mathrm{~cm}}-$ the inverse Plank's length. The large number for $\mathbf{G}$ is the reason for the small value of Newton Gravitational constant $K_{N}$, which is inverse proportional to the square of amplitude of the Gravitational vector field $\mathbf{G}\left(K_{N}=\frac{c^{3}}{G^{2} \hbar}\right)$. At large distances from the gravitational source $G^{2}$ has this dependence with respect to the distance $\rho$ and the value of $\mathbf{G}$ vector at infinity $\left(G_{\infty}^{2}\right)$ :

$$
G^{2} \approx\left(G_{\infty}\right)^{2}+\frac{N}{L_{a} \rho} \text { or } K_{N} \approx \frac{c^{3}}{\hbar\left(G_{\infty}\right)^{2}}\left[1+\frac{N}{\left(G_{\infty}\right)^{2} L_{a} \rho}\right]^{-1}
$$

where $N$ is number of particle (or effectively the mass of the source) and $L_{a}$ is atomic length.

This relation between Newton gravitational constant and the Gravitational vector, or dependence Newton Gravitational constant upon the distance could be the basis of both Pioneer anomaly and the abnormal star rotation in some Galaxies. As distance increases the Newton Gravitational constant increases, which in effect increases the attraction toward the center. In our solar system the 
effect is small and looks like "extra pull" by the Sun toward its center, which reduces the speed of a satellite. In some Galaxies where $N$ is large the effect could be quite significant to alter the rotation periods of the stars as a function of their distances from the central source. See Section 3-Introduction.

2) We begin building the theory using Affine Unification approach thru nonsymmetrical affine connections $\Gamma$, but not as a geometrical description, but rather as axiomatic one. We define tensor of Total-Matter $\left(\mathbf{M}^{\mathrm{T}}\right)$ as "generalized" Riemann tensor and we define the Lagrangian density $L^{\checkmark}$ of the Total-Matter as a function of the tensor $\mathbf{M}^{\mathrm{T}}$ only. The equations of motions for $\Gamma$ are obtained by means of variations with respect to $\Gamma$ (see Section 4-Affine Unification):

$$
S=\int \mathrm{d} x^{4} L^{\sqrt{ }}\left(\mathbf{M}^{\mathrm{T}}(\boldsymbol{\Gamma})\right)=>\frac{\delta L^{\sqrt{ }}}{\delta \boldsymbol{\Gamma}}=0 .
$$

The most interesting and perhaps the most suitable are these two Lagrangians of the 4th power:

$$
\begin{aligned}
& S=\int \mathrm{d}^{4} x L^{\sqrt{ }} \quad \text { where } L^{\sqrt{ }}=\sum_{s=\text { inv }} \lambda_{s}\left[\mathbf{M}^{\mathrm{T}}(\boldsymbol{\Gamma})\right]^{4}\left[\mathbf{M}_{(R i)}^{\mathrm{T}}(\boldsymbol{\Gamma})\right]^{-4} \sqrt{\mid \operatorname{det}\left[\mathbf{M}_{(R i)}^{\mathrm{T}}(\boldsymbol{\Gamma})\right]} \\
& \quad \text { or } L^{\sqrt{ }}=\sum_{s=\text { inv }} \lambda_{s}\left[\mathbf{M}^{\mathrm{T}}(\boldsymbol{\Gamma})\right]^{4} \boldsymbol{\Delta}_{M}^{2} \sqrt{\left|\operatorname{det}\left[\mathbf{M}_{(R i)}^{\mathrm{T}}(\boldsymbol{\Gamma})\right]\right|} ; \quad \boldsymbol{\Delta}_{M}=\frac{\Delta^{i j k l}}{\sqrt{\operatorname{det}\left[\mathbf{M}_{(R i)}^{\mathrm{T}}(\boldsymbol{\Gamma})\right]}}
\end{aligned}
$$

where $\boldsymbol{\Delta}_{M}$ is Levi-Chivita tensor, $\sum_{s=\text { inv }}$ indicate the summation of all possible invariants with a constant $\lambda_{s}$ coefficient associated with each particular invariant.

3) We then define space metric $\mathbf{r} \equiv r_{i j}$ based on the elimination of some components of the Tensor-Potential $\mathbf{P} \equiv P_{j k}^{i}$ defined as $P_{j k}^{i}=\Gamma_{j k}^{i}-\gamma_{j k}^{i}(r)$. In general $\mathbf{P}$ consists of 64 components and could be split on 4 vectors and 3 traceless 3-index tensors-one fully symmetrical $\mathbf{N} \equiv N_{i j k}$ and two torsion type tensors $\mathbf{K} \equiv K_{i j k}=-K_{i k j}$ and $\mathbf{C} \equiv C_{i j k}=-C_{i k j}$ each of which has only 16 independent components.

$$
\begin{aligned}
& N_{i j k} r^{i j}=0 ; \quad K_{i j k}=-K_{i k j} ; \quad K_{i j k} r^{i j}=0 ; \quad K_{i j k} \frac{\Delta^{i j k l}}{\sqrt{\left|\operatorname{det}\left(r_{i j}\right)\right|}}=0 \\
& C_{i j k}=-C_{i k j} ; \quad C_{i j k} r^{i j}=0 ; \quad C_{i j k} \frac{\Delta^{i j k l}}{\sqrt{\left|\operatorname{det}\left(r_{i j}\right)\right|}}=0
\end{aligned}
$$

where $\frac{\Delta^{i j k l}}{\sqrt{\left|\operatorname{det}\left(r_{i j}\right)\right|}}$ is Levi-Chivita tensor.

Our definition of metric $\mathbf{r}$ is based on requirement that two torsion tensors are proportional to each other: $\mathbf{K}=\mu_{0} \mathbf{C} \quad\left(\mu_{0}\right.$-constant).

This procedure makes the metric $\mathbf{r}$ a dynamic variable of the theory and transfers the Affine description to Tensor-Potential description over curved space with metric $\mathbf{r}$. See Section 5-Tensor Potential.

4) The general form of the Lagrangian of the Total-Matter can be written thru the combination of invariants of the the Total-Matter $\mathbf{M}^{\mathrm{T}}$ and thru the con- 
stants $\lambda_{n} s$ associated with each invariant:

$$
\begin{aligned}
& S=\int \mathrm{d}^{4} x L^{\sqrt{ }} \equiv \int \mathrm{d}^{4} x \sum_{n=1, \cdots} L_{n}^{\sqrt{ }} \\
& \text { where } L_{n}^{\sqrt{ }}=\sum_{s=\text { inv }} \lambda_{n s}\left[\mathbf{M}^{\mathrm{T}}\left(\mathbf{P}^{\mathrm{T}}\right)\right]^{n}\left[\mathbf{M}_{(R i)}^{\mathrm{T}}\left(\mathbf{P}^{\mathrm{T}}\right)\right]^{-n} \sqrt{\mid \operatorname{det}\left[\mathbf{M}_{(R i)}^{\mathrm{T}}\left(\mathbf{P}^{\mathrm{T}}\right)\right]} .
\end{aligned}
$$

5) The determination of the constant parameters $\lambda_{n s}$ as well as maximum " $n$ " is done by the way of reducing the above Lagrangian to the Lagrangian of the "small system"-the system for which its own Gravitational field in much smaller then the Gravitational field at infinity as viewed from point of view of the "small system". In the end of this procedure the Lagrangian of the "small system" should have a typical field-theory form. During this derivation there will be some "unwanted" terms, which must vanish. The requirements for vanishing these terms should be satisfied by the choice of the $\lambda$-constants in front of invariants that form the Lagrangian.

6) We show that utilizing the above procedure, the Lagrangian of the "small system" (or weak Gravitational field), as it is in our Solar System, can be simplified to have this form:

$$
S=\int \sqrt{-r} \mathrm{~d}^{4} x L^{\sqrt{ }} \approx \int \mathrm{d}^{4} x \bar{L} ; \quad \bar{L}=\bar{L}^{(M)}+\frac{\bar{L}^{(\text {Grav })}}{\left(G_{\infty}\right)^{2}}
$$

where $\bar{L}^{(M)}$ represents the Lagrangian of the "typical" Matter in flat Minkowski space and $\bar{L}^{\text {(Grav) }}$ corresponds to Lagrangian that describes the Gravitation thru vector $\overline{\mathbf{G}}$ and the metric $\overline{\mathbf{r}}$-both (labeled as bar functions) being corrections to a constant Gravitational field at infinity $G_{\infty}$ and flat Minkowski space correspondingly. See Section 6-Lagrangian-expr. (60).

7) One of the feature of the existence large Gravitational field $\mathbf{G}$ is that both Lagrangian of the Matter $L^{(M)}$ and of the Gravitation $L^{(\text {Grav })}$ include a unit vector $\mathbf{U}$ of the Gravitation vector $\mathbf{G}\left(U_{i}=G_{i} / \sqrt{G_{i} G^{i}}=(1,0,0,0)\right)$. This fact allows the equations of motion for some variables (in fact most variables) to be not hyperbolic $\left(\partial_{t t}-\partial_{x x}\right)$ as Minkowski metric demands, but elliptical $\left(\partial_{t t}+\partial_{x x}\right)$, which by its nature forbids the existence independent waves and their propagation. In elliptic equations the field's time dependence is such that the field modifies itself (thus will have time depending behavior), but it quickly decays to zero any king of oscillating harmonics that it produces. Applied to the description of Gravitation, it would state that there are no Gravitational waves, which is supported by the results of long time LIGO program in US and around the world.

8) In the weak gravitational fields the $L^{(\text {Grav })}$ provides all the information needed for description of either vector $\mathbf{G}$ and metric $\mathbf{r}$ in both vacuum and inside the matter. It also contains the terms corresponding to Grav-field to metric interaction. However as we showed, in a specific system of coordinate this interaction vanishes and metric becomes depending only on the matter that produced it (i.e. its deviation from the flat space). This might support the Einstein idea that Gravitation can be described as a curved space and it alone governs 
the "classical body" movement.

The special system coordinate which is equivalent to the "rest" system of coordinates is defined as the one where metric (or its correction $\overline{\boldsymbol{r}} \equiv \bar{r}_{i j}$ ) satisfies the first order derivatives relations-the "gauge":

$$
\begin{aligned}
& \bar{r}_{i k, m} \delta^{k m}=\alpha_{1} \bar{r}_{, i}+\alpha_{2} \bar{r}_{m i, n} \bar{U}^{m} \bar{U}^{n}+\alpha_{3} \bar{r}_{m n, i} \bar{U}^{m} \bar{U}^{n}+\alpha_{4} \bar{r}_{m n, s} \bar{U}^{m} \bar{U}^{n} \bar{U}^{s} \bar{U}_{i}+\alpha_{5} \bar{r}_{, m} \bar{U}^{m} \bar{U}_{i} \\
& \text { or } \bar{r}_{i k, m} \delta^{k m}=\alpha_{1} \bar{r}_{, i}+\alpha_{2} \bar{r}_{0 i, 0}+\alpha_{3} \bar{r}_{00, i}+\alpha_{4} \bar{r}_{00,0} \bar{U}_{i}+\alpha_{5} \bar{r}_{,} \bar{U}_{i} \\
& \text { where } \bar{r}=\bar{r}_{i j} \delta^{i j} ; \quad \bar{U}_{i}=(1,0,0,0) \quad \delta_{i j}=\operatorname{diag}(1,-1,-1,-1)
\end{aligned}
$$

and all $\alpha$-s are the constants defined by the $\lambda$-constants which define the form of Lagrangian-see item 4) above as well as expr. (85). See Section 7- Equations for Gravitational Field and Metric.

9) In the case of weak Gravitational field (as in our Solar system) for the description of Electromagnetic field (or to be more accurate its vector potential) $\mathbf{E} \equiv E_{i}$ this theory derives the Lagrangian $L^{(E)}$ in this form:

$$
\begin{aligned}
& L^{(E)}=\stackrel{M}{i j}_{i j} E_{k l} \delta^{i k} \delta^{j l}+E_{i} E_{j} F_{k l}^{(E)} \delta^{i k}+E_{i} J_{k}^{(E)} \delta^{i k} \\
& \text { where } \delta_{i j}=\delta^{i j}=\operatorname{diag}(1,-1,-1,-1) ; \quad E_{i j}=E_{i, j}-E_{j, i}
\end{aligned}
$$

which differs from the Maxwell Lagrangian by the presence of the mass-photon term $\mathbf{E}^{2} \mathbf{F}^{(E)}$, where the 2-index tensor $\mathbf{F}^{(E)}$ is a short distance function associated with and defined by the localized mass-matter (or particle such as proton, electron, etc.) and thus is zero outside of mass-matter. The assymptotics at large distances $\rho$ should still be $Q^{(e)} / \rho$ with $Q^{(e)}$ being a constant, which for the a single particle would lead to a charge defined as:

$$
Q^{(e)}=\int_{\text {all space }} \mathrm{d} x^{3}\left[J_{0}^{(E)}+E_{i} F_{0 j} \delta^{i j}\right]
$$

which by the proper "normalization" procedure must be set to \pm 1 . As long as the asymptotics at infinity is still $Q_{e} / \rho$, the Maxwell description of the Electromagnetic field of a macro system (in statistical sense) of charged particles (as long as they far enough away from each other and probably moving with the speed much less than the speed of light) could be recovered by introducing Dirac's $\delta$-function as a flux.

$$
\text { or } \quad \stackrel{\text { Max }}{E_{i j}} \delta^{j k}=J_{i} ; \text { where } J_{i}=\sum Q^{(e)} u_{i} \delta(\mathbf{r})
$$

where $u_{i}$ 4-dimensional vector velocity. In that sense the standard Maxwell equations for the Electromagnetic field should be viewed as an approximation of more complex equations above derived thru Affine Unification. See Section 8Electromagnetic and Other Fields.

10) The proposed theory also suggests the existence of two other vector fields D and $\mathbf{B}$. The first one, $\mathbf{D}$ is described by Maxwell type equation, but with a flux corresponding not to a charge particle but to a dipole (zero total charge).

$$
\begin{aligned}
& L^{(D)}=\stackrel{\operatorname{Max} \operatorname{Max}}{D_{i j} D_{k l} \delta^{i k} \delta^{j l}+\stackrel{\operatorname{Max}}{D_{i j}} F_{k l}^{(D)} \delta^{i k} \delta_{j l}} \quad \text { where } \stackrel{\text { Max }}{D_{i j}}=D_{i, j}-D_{j, i} \\
& \text { and } \quad \delta_{i j}=\delta^{i j}=\operatorname{diag}(1,-1,-1,-1) ; \quad Q^{(e)} \equiv \int_{\text {all space }} \mathrm{d} x^{3} J_{0}^{(D)}=\int_{\text {all space }} \mathrm{d} x^{3} F_{0 i, k}^{(D)} \delta^{i k}=0
\end{aligned}
$$


The D -photon has zero mass everywhere (both vacuum and inside the massmatter) and its interaction with mass-matter is such that it only can exchange the energy-very much similar to a neutrino. See Section 8-Electromagnetic and Other Fields; see after expr. (132).

11) Derived from Affine Unification the description of $\mathbf{B}$ vector field, suggest the existence of short-distance, strong-interactive with mass-matter and highly non-linear particle (like Bozons), whose Lagrangian in vacuum contains terms proportional to the forth power of B. See Section 8-Electromagnetic and Other Fields; see text starting at expr. (150).

12) Proposed theory provides an avenue of describing the micro world (massparticle like proton and electron) from field point of view and thus to offer alternative approach to the Quantum Theory, or at least, offers a classical approximation and a smooth transition from one classical description of the matter to the quantum mechanical one. In this theory the mass-matter is described by one fully symmetrical 3-index tensor $\mathbf{N}$ and one torsion-type 3-index tensor $\mathbf{C}$. The Lagrangian of the mass-matter is highly non-linear (4th power) with respect to the tensors $\mathbf{N}$ and $\mathbf{C}$, which leads to the highly localized solution. The Einstein formula $E=m c^{2}$ is in a effect a "normalization requirement" for the constants of integrations for $\mathbf{N}$ and $\mathbf{C}$. It would make sense to assume (or to require) that the equations for $\mathbf{N}$ and $\mathbf{C}$ are described by an elliptical equations (which only possible due to existence of the Gravitational Unit vector $\mathbf{U}$ ), thus corresponding to (or providing) the mass-matter particle's stability. See Section 9-Mass-Matter.

13) In proposed theory the Cosmology of Universe naturally leads to oscillating Universe. As Universe expands the average Gravitational field decreases, the effective value of Newton's Gravitational constant increase, which increases gravitational pull of masses. Eventually, this pull will be strong enough to stop the expansion and reverse it to compression of the Universe. At the other end the shrinking of the size of Universe increases the average value of the gravitational field $\mathbf{G}$, effectively decreasing the Newton's gravitational constant and thus decreasing gravitational pull of masses. The mass' kinetic energy (the temperature of Universe) would be large enough to stop contraction of the Universe and reverse it back to expansion. Thus leading to the eternal oscillation of the Universe.

Its mathematical form (even with assumption of uniform distribution of matter in the Universe) is much more complex since it cannot use a "small system" Lagrangian, but must use the exact Lagrangian of the Total Matter, which is (at least) of forth power with respect to Grav-field $\mathbf{G}$ (or more accurate $\mathbf{G}^{\prime}$ ), space curvature (Riemann metric tensor $R_{i j k l}$ ) and the regular Matter. And in order to find (fully define) such Lagrangian - to find all the $\lambda$-s and the parameter " $n$ "-we must execute the program described in this paper.

Because in proposed theory the Gravitation is described only thru atomic parameters-i.e. $\hbar$ as a measure of energy and mass, $c$ being the speed of light and $L_{a}$ being the atomic length - and the number of particle in the Universe, it is not difficult to see that the scale of the basic parameters of the Universe-the 
radius of Universe $L_{U}$ (measures in atomic length $L_{U} / L_{a}$ ) and the Time-Scale of the Universe $T_{U}$-are given by a simple relation with respect to the number of particles in the Universe $N$, where both of basic Universe parameters are proportional to a square root of the number of particles: $L_{U} / L_{a}=T_{U} c / L_{a}=\sqrt{N}$, with $N \approx 10^{83}$.

\section{Definitions}

General convention:

a) Capital Latin letters represent unknown functions. Capital "bold" Latin letters (except for $\mathbf{r}$ ) represent a tensor. For example $\mathbf{E}$ is a vector field $\left(\mathbf{E} \equiv E_{i}\right), \quad \mathbf{P}$ is a 3-index tensor $\mathbf{P} \equiv P_{j k}^{i}$ and $\mathbf{r} \equiv r_{i j}$ is a metric tensor;

b) Non-capital Latin letters (typically $i, j, k, 1, m, n$ ) used as index take values $0,1,2,3$;

c) Non-capital Greek letters are constants; For example, $\lambda$-constants in definition of the Lagrangian $\mathrm{L}$ in general, $\mu$-in definition of Matter $\mathbf{M}, \sigma-$ in definition of Lagrangian of the square-vector $\mathbf{S}, \pi$-in definition of tensor Potential P;

d) Non-capital Greek letters used as an index take value " $x$ ", “ $y$ ", " $z$ " in flat Minkowski space;

e) Capital Latin letters used as index (usually in parentheses and usually upper index) identifiers of the group of functions (such as Electromagnetic field, etc.). For example: $L^{(E)}$ refers to Lagrangian of the vector field E.

1) $K_{N}$-Newton gravitational constant; $L_{a}=\hbar c / m c^{2}$-atomic length.

2) $\mathbf{r} \equiv r_{i j}-$ metric tensor; $\delta_{i j}=\operatorname{diag}(1,-1,-1,-1) ; \delta^{i j}=\operatorname{diag}(1,-1,-1,-1)-$ flat Minkowski metric in Descartes coordinates. $\delta_{j}^{i}=\operatorname{diag}(1,1,1,1)-$ Kronecker symbols.

$\Delta \equiv \Delta_{i j k l}=(-1)^{p}$-fully antisymmetric symbols, where $p$ is a permutation number from " 1234 ".

$\Delta_{a} \equiv \frac{1}{\sqrt{\left|\operatorname{det}\left(a_{i j}\right)\right|}} \Delta_{i j k l}$-Levi-Civita fully asymmetrical tensor based on the symmetric tensor $a_{i j}$.

3) $\gamma_{j k}^{i} \equiv \frac{1}{2} r^{i m}\left(r_{m j, k}+r_{m k, j}-r_{j k, m}\right)$-Christoffel symbols.

$\mathbf{R} \equiv R_{j k l}^{i}=\gamma_{j l, k}^{i}-\gamma_{j k, l}^{i}+\gamma_{m k}^{i} \gamma_{j k}^{m}-\gamma_{m l}^{i} \gamma_{j k}^{m}-4-D$ (time-space) Riemann tensor built on $\gamma_{j k}^{i}$.

$\Gamma \equiv \Gamma_{j k}^{i} \neq \Gamma_{k j}^{i}$-non-symmetrical Affine Connections.

$\mathbf{M}^{\mathrm{T}}$ - tensor of the total Matter defined thru Affine Connections $\Gamma$ (see expression 9). For example, $\left(M^{\mathrm{T}}\right)_{j k l}^{i} \equiv \Gamma_{j l, k}^{i}-\Gamma_{j k, l}^{i}+\Gamma_{j m}^{i} \Gamma_{j l}^{m}-\Gamma_{m l}^{i} \Gamma_{j k}^{m}$.

$$
\mathbf{M}_{(R i)}^{\mathrm{T}} \equiv \frac{1}{2}\left[\left(M^{\mathrm{T}}\right)_{j k l}^{k}+\left(M^{\mathrm{T}}\right)_{l k j}^{k}\right] \text {-symmetric "Ricci" tensor for } \mathbf{M}^{\mathrm{T}} .
$$

$\mathbf{P}^{\mathrm{T}} \equiv\left(P^{\mathrm{T}}\right)_{j k}^{i}=\Gamma_{j k}^{i}-\gamma_{j k}^{i}$-Potential defined by $\Gamma$ and metric $\mathbf{r}$.

$\mathbf{G}$-vector field; $\mathbf{G} \equiv G_{i} ; G=\sqrt{\left(G_{i} G^{i}\right)} ; G_{\infty}$-value of the vector field at infinity $\mathbf{U} \equiv U_{i}=G_{i} / G$ - unit vector of vector $\mathbf{G} \quad \mathbf{S} \equiv S_{i}=G G_{i}$-square-vector. 
$\mathbf{P} \equiv P_{j k}^{i}-$ Tensor-Potential defined as $\mathbf{P}^{\mathrm{T}}$ without the field $\mathbf{G}$.

$\mathbf{M}$ is the tensor of Matter defined as a tensor of Total-Matter without gravitational field $\mathbf{G}: \mathbf{M}=\left.\mathbf{M}^{\mathrm{T}}\right|_{\mathbf{G}=0}$.

$\mathbf{E}^{*}$-Vector field defined thru a fully antisymmetric tensor $\frac{\Delta_{i j k l} E^{l}}{\sqrt{\left|\operatorname{det}\left(r_{m n}\right)\right|}}$. $\mathbf{E}^{\prime *}=\left(E_{k, l}-E_{l, k}\right) \frac{\Delta_{i j k l} E^{l}}{\sqrt{\left|\operatorname{det}\left(r_{m n}\right)\right|}}-$ Maxwell “star" tensor.

$\mathbf{J}$-one-index tensor $\left(\mathbf{J} \equiv J_{i}\right)$.

$\mathbf{F}$-two-index tensor $\left(\mathbf{F} \equiv F_{i j}\right)$.

$\mathbf{N}$-3-index fully symmetrical traceless tensor $\left(\mathbf{N} \equiv N_{i j k} ; N_{j k}^{j}=0\right)$.

$\mathbf{C}-3$-index traceless torsion type tensor $\left(\mathbf{C} \equiv C_{i j k}=-C_{i k j}\right)$.

$C_{j k}^{j}=0 ; C_{i j k} \Delta^{i j k l}=0 ; \Delta \equiv \Delta_{i j k l}=(-1)^{p}$-fully anti-symmetric symbols.

$\mathbf{K}$-3-index traceless con-torsion type tensor $\hat{C}_{i j k}=\hat{C}_{i k j} \equiv C_{j i k}+C_{k i j}$.

$\mathbf{H}$-symbolic writing of a pair 3-index tensors $\mathbf{N}$ and $\mathbf{C}: \mathbf{H} \equiv(\mathbf{N}, \mathbf{C})$.

$L^{\sqrt{ }}$-Lagrangian density defined as $L^{\sqrt{ }}=L \sqrt{\left|\operatorname{det}\left(r_{i j}\right)\right|}$.

4) All "bar" functions are tensors defined over flat Minkowski space. All covariant derivatives replaced by partial ones. The indeces are raised by Minkowski metric $\delta^{i j}=\operatorname{diag}(1,-1,-1,-1)$.

$$
r_{i j}=\delta_{i j}+\bar{r}_{i j} ; \quad S_{i}=\left(G_{\infty}^{2}\right)_{i}+\bar{S}_{i} ; \bar{U}_{i}=(1,0,0,0) \quad \bar{\gamma}_{i j k}=\frac{1}{2}\left(\bar{r}_{i j, k}+\bar{r}_{i k, j}-\bar{r}_{j k, i}\right) .
$$

\section{Introduction}

The question why gravitational force is so small in comparison to nuclear forces has been a major puzzling question in physics for the last hundred years. If one accepts that gravitation is a fundamental force one must conclude that there must be "particles" (or non trivial physics) with a size of Plank's length $10^{-33} \mathrm{~cm}$, which is some 20 orders of magnitude smaller than the expected size of a proton (nuclei) - which is about the same ratio as the size of a proton to the size of the Earth (or even better comparison: as a size of a tennis ball to the size of the Universe). This disparity in sizes leads to a logical question whether gravitation is an independent (fourth) interaction or an emergent phenomenon, that is to say that gravitation could be explained by means of atomic interaction and statistical analysis of large number of particles. And if the latter is true than the Gravitational constant is not universal constant, but a value of a function at a given point. This means that in describing gravitation we have to consider not only metric (as it has been done by Einstein in GR), but, in addition, a function (or some functions), that could be viewed as the gravitational matter-or as commonly referred to as "dark matter".

This is not totally new idea. Some 50 years ago Brans-Dicke offered function-metric theory of gravitation [1] (also see [2]), with a hope to resolve the problems of General Relativity, in which the "small gravitational constant" is not the only one. Philip D. Mannheim in his "Shortcomings of Einstein Gravity" [3] very eloquently describes the issue and the necessity of modification of GR, which also expressed in the fact that probably as much as $25 \%$ of all papers on 
gravitation (based on arXiv.org/General Relativity and Quantum Cosmology) are written for that purpose in mind: “... we need to ask whether the Einstein theory is in fact the only theory which then meets the three classic tests. Beyond this, we also note that when Einstein gravity is extended beyond its solar system origins, no matter in which way it is extended additional concerns arise. When Einstein gravity is extended to galactic distance scales we get the dark matter problem. When Einstein gravity is extended to cosmological distance. With regard to singularities, not only are there no data which provide direct evidence for the existence in nature of event horizons or trapped surfaces (or even whether the mass concentrations in galactic centers have radii less than their Schwarzschild radii), it is not clear whether the existence of singularities in the fabric of spacetime is a property of nature or an indication of the breakdown of the theory. Finally, to resolve the renormalizability problem it has been found necessary to generalize the theory to a superstring theory which introduces two further ingredients for which there is also no experimental evidence, namely the existence of ten spacetime dimensions and the existence of a supersymmetry which gives all known particles as yet undetected superpartners".

The necessity of Newton's gravitational constant being a part of the equations of General Relativity and its generalizations [4]-[17] steams from the fact that the space-time curvature Ricci tensor $R_{i j}$ has units $1 /(\text { length })^{2}$ and the tensor of Energy-Momentum expressed in the units $\hbar c$ has units $1 /$ (length) ${ }^{4}$. In order to remove this problem the Ricci tensor must be multiplied by a function with units $1 /$ (length) $)^{2}$. The simplest such function is a square of a vector's magnitude. Since $G_{i} d x^{i}$ is invariant, then $G_{i}$ is measured in units $1 /$ (length) and the square of its magnitude is measured in units $1 /$ (length) ${ }^{2}$. With this in mind we can modify the Einstein's equations of General Relativity by replacing gravitational constant with a square of magnitude of the Gravitational field and by adding a Lagrangian $L_{G}$ of dark matter (which we limit to only 3 terms, intentionally omitting non-linear with respect to $G$ terms-like $\left.G_{k ; l} G_{m ; n} r^{k m} G^{l} G^{n} / G^{2}\right)$ and writing the action integral in the following manner:

$$
\begin{aligned}
& S=\int \mathrm{d}^{4} x\left(\hbar c G^{2} R+L_{M}+L_{G}+L_{G M}\right) \sqrt{-\operatorname{det}\left(r_{i j}\right)} \\
& L_{G}=\hbar c\left[\lambda_{1} G_{k ; l} G^{k ; l}+\lambda_{2} G_{k ; l} G^{l ; k}+\lambda_{3}\left(G_{k ; l} r^{k l}\right)^{2}\right]
\end{aligned}
$$

where $r_{i j}$ is a space metric, $G_{k}$ is a vector field, $G=\sqrt{G_{k} G^{k}}$ and $\lambda$ 's are some constants. $L_{M}$ is a Lagrangian of actual physical matter and $L_{G M}$ is the term responsible for dark matter interaction with the physical matter, which typically is assumed to be zero. This, in a way, is a combination of twoBrans-Dicke [1] and Vector-Metric [18] - theories for description of dark matter. And comparing the above expression with GR we conclude that the value (at least in our solar system) of the vector G magnitude is inverse to the Plank's length and given by the following expression:

$$
G=\sqrt{\frac{c^{3}}{K_{N} \hbar}}=\frac{1}{L_{p}} \approx 10^{33} \frac{1}{\mathrm{~cm}} .
$$

Even though this Lagrangian contains no Gravitational constant, it-as we 
will show in this paper-has some major problems in vector description of of Dark Matter G-see comment after expr. (40) - and its dark-matter part $\left(L_{G}\right)$ must be replaced (which we will do) by a correct one. However, in principle, it give a correct physical description of the problem.

From the expression above follows that the gravitational constant is inverse proportional to the square of magnitude of the gravitational vector defined by the particles of matter (protons and electrons). In our solar system magnitude of the gravitational vector field is mostly defined by the matter outside of solar system-Milky Way Galaxy or/and Universe-and thus almost constant within the solar system. The presence of solar matter (mostly of course is the mass of Sun) gives a small addition to the magnitude of grav-field and, as we will show in this paper, could be written in the following manner:

$$
\begin{aligned}
& K_{N}=\frac{c^{3}}{\hbar G_{\infty}^{2}} \\
& G^{2}=G_{\infty}^{2}+\frac{N_{s}}{L_{a}} \frac{1}{\rho} \quad L_{a}=\frac{\hbar c}{m_{p}}
\end{aligned}
$$

where $\rho$ is a distance from the Sun, $N_{s}$ is the number of baryons of the Sun $\left(N_{s} \approx 10^{57}\right), L_{a}$-atomic length of a protons $\left(L_{a}=10^{-13} \frac{1}{\mathrm{~cm}}\right)$ and $K_{N}$ is the Newton's gravitational constant.

Using the above formula, we can try to explain the "pioneer anomaly" [19]. Based on a measurement of the communication frequency of the Pioneers' satellites it had been concluded the the Pioneer 10 and 11 on their trajectories out of our Solar system were experiencing a slow-down (small fraction of their velocity) as if they had stronger attraction toward the Sun. This could be explain by vector metric theory of gravitation and the formula above that it produces. As distance from the Sun increases, the Grav-field $\left(G^{2}\right)$ decreases, which corresponds to increase Newton's gravitational constant $K_{N}$. That in effect corresponds to increase of attraction toward the Sun. Eventually, the velocity of the satellite is going to stabilized, but at somewhat smaller value than the expected value based on $K_{N}$ in vicinity of Sun.

The same mechanism can be used to explain the abnormality of some stars rotation in some galaxies [20] [21] [22]. Per Newton theory the square of star's velocity is inverse proportional to the distance of that star to the center of its Galaxy. In reality the experimental data shows that the velocity of is almost constant. This could be easily explained by vector-metric theory of gravitation thru gravitation constant dependence on distance.

$$
\begin{aligned}
& G^{2}=G_{\infty}^{2}+\frac{N_{\mathrm{gal}}}{l_{a}} \frac{1}{\rho}=G_{\infty}^{2}+\frac{c M_{\mathrm{gal}}}{\hbar} \frac{1}{\rho} \text { where } l_{p}=\frac{\hbar}{m_{p} c} \\
& K_{N} \equiv \frac{c^{3}}{G^{2} \hbar}=\frac{c^{3}}{G_{\infty}^{2}+\frac{c M_{\mathrm{gal}}}{\hbar} \frac{1}{\rho}} \\
& V_{\mathrm{star}}^{2}=\frac{K_{N} M_{\mathrm{gal}}}{\rho}=\frac{c^{3} M_{\mathrm{gal}}}{\hbar G_{\infty}^{2} \rho+c M_{\mathrm{gal}}}
\end{aligned}
$$

Unlike in our Solar system where "small" mass of our Sun is responsible for a 
tiny change of gravitational constant, in some Galaxies, the dependence of gravitational constant on distance could be quite significant. More so, if the value of gravitational field at infinity is defined by Universe and its value is much less than the Grav-field created by the center of Galaxy, then there is a good possibility that going from one Galaxy to the other, the gravitational field (and thus the gravitational constant) could vary significantly-in large number of per-cent if not an order of magnitude.

As to the origin of the gravitational field it reflects the accumulative effect of all the particles in Universe (or at least in our Galaxy). The gravitational field (as we will see it later) is an attribute of the particle (proton, electron) just as electric field is an attribute of the charge. But unlike the electrical field it is not canceled, but is accumulated leading to it's enormous value $G \approx 10^{33} \mathrm{~cm}^{-1}$; And it is this large value is responsible for the small effect of gravitation on any sub-system (even as big as our Sun) of the Universe.

The idea that "universal" large numbers-such as ration between electric forth of two protons and their Gravitational forth, which is about $10^{36}$-are somehow related to the number of particle in Universe has long been proposed by Weyl, Eddington and Dirac [23] [24] [25] [26] [27].

There are few immediate mathematical ramification of having large Gravitational field, which comes from the main postulate of Modern physics (and experiment) that law of physics for any isolated system are identical.

If we consider a "small matter" ( $\mathbf{m})$ in a vacuum and far away from other bodies, then the Lagrangian of the "small matter" should be derived from the Lagrangian of the Total-Matter by ignoring all other matters and thus be a function of only "small matter", space metric and the total Gravitational field: $L^{(m)}=L^{(m)}(\mathbf{G}, \mathbf{m}, \mathbf{r})$, where $\mathbf{G}$ is the total Grav-vector consisting of Grav-vector of the "small matter" $\left(\mathbf{G}^{(m)}\right)$ added to the Grav-field of all other "outside" Matter $\mathbf{G}_{\infty}: \mathbf{G}=\mathbf{G}_{\infty}+\mathbf{G}^{(m)}$.

If we assume that $L^{(m)}$ includes the magnitude of Grav-field $\mathbf{G}$ then, the solution for a "small Matter" will include very large parameter $G_{\infty} \approx 10^{33} \frac{1}{\mathrm{~cm}}$ (in our solar system) and thus would have its property (mass, charge, etc.) depend on this large number-which of course is not the case.

And if we don't want the Plank's length (which is $\approx 1 / G_{\infty}$ ) enter into the equations of Matter, we must postulate that the Lagrangian of the Matter $L^{(m)}$ can not depend on magnitude of $\mathbf{G}$, but only on its direction $\mathbf{U}$ or it's derivative $\mathbf{G}^{\prime}$ at least in the first order of parameter $\mathbf{G}$ :

$$
L^{(m)}=L^{(m)}\left(\mathbf{G}^{\prime}, \mathbf{m}, \mathbf{U}, \mathbf{r}\right) .
$$

This physical picture could be compared to the description of body on the top of the ocean of water. It too does not include the depth of the ocean. The body only "sees" the result of the changing of water, which is comparable (in size) to the size of the body.

We will use this requirement in the following section when we will examining 
the form of thew Lagrangian of the Total-Matter.

There is one more consideration-and that is Lawrence invariant-that need to be addressed here. Since we use a tensorial apparatus the equations of motions and all invariants are independent of coordinate transformation. In that sense introduction of additional vector field does not produce any problems. However, if we consider the Lawrence invariance in more narrow sense-form of equation in the small area where metric tensor might be considered Minkowski-then the equations of Matter might (it does not have to, but it might) depend on the Gravitational vector $\mathbf{G}$ or at least on its direction $\mathbf{U}$. From philosophical point of view, regardless whether such dependence on Grav-vector $\mathbf{G}$ (or its unit vector $\mathbf{U}$ ) is explicitly present or not in the equations of motion, the existence of Grav-vector $\mathbf{G}$ by itself yields the existence one "special" system of coordinates that could be called the "rest" system of coordinates. The coordinates in which Grav-vector $\mathbf{G}$ has only time component: $\mathbf{G}=(1,0,0,0)$.

V. Fock [28] long time ago argued that such system of coordinate must exist. Without it (as it is in case of GR) one cannot say if Earth moves around Sun or vise versa. The Newton equations might be invariant in any inertial system of coordinate, but we are absolutely sure that the Universe is fixed and it is we that move. And not other way around.

This paper could be called "vector-metric theory of gravitation", which could've started with postulating-instead of the Lagrangian (1) above-the correct Lagrangian for the grav-vector $\mathbf{G}$. However, it would look totally at hog and without any justification. It would also leave an opened question about the expression for $L_{G M}$, which-as we will show in this paper-does not have a "simple" answer in the phenomenological approach above, but it does have a clear answer, if we consider a more general theory of Affine Connections as a source for derivation of the vector-metric theory of gravitation.

By opening the scope of this paper to the level where both Gravitation and Electromagnetism can be derived from more general considerations, we are able not only to derive the expressions for $L_{G M}$, but to answer some unclear questions such as a) is the tensor of Energy-Momentum the "source" in description of gravitations as Einstein's GR postulate? b) are Einstein's equations fundamental or phenomenological (approximations); and if the latter is true, what is their limits-meaning can they be used for description of Universe c) is there a rest system coordinates?

Also we will derive the expression for a Lagrangian that could be used for description of any system of particles-as small as atoms or as large as our Sun-as long as its Gravitational field can be treated as "small" in comparison to Gravitational field at infinity. And by doing so we will also be able to see dependence the Lagrangian $L_{M}$ has on the gravitational vector.

For the majority of physicists who undoubtedly reject Eddington's Unification idea strictly on the grounds that true description of matter must be Quantum Mechanical, may I suggest two alternative points for considerations. First, one can view Eddington's description as a classical limit of quantum mechanical 
wave functions, as it is in of electromagnetism, where electromagnetic field is a classical representation of quantum photons. And second, one can use Eddington's formalism as a starting point for quantization as its done for bozon quantum theory, which starts as a classical non-zero mass vector field [29].

Here, it would be a proper place to declare author's point of view that had been a main belief and a driving force in the work that is presented in this paper: the quantum mechanical theory is our attempt to express rather complicated and strictly non linear field description of matter through a linear formalism of wave functions. In its essence this is the Einstein point of view in his famous and now classical debate with Neil Bohr on the meaning of wave function.

\section{Affine Unification}

Eddington's idea of Unifications over almost 100 years of its history took different forms and modifications [31] geared for different applications and purposes [32]-[36]. However, in its original and most general form the Eddington's theory of Unification [30] is characterized as universal attempt to geometrisize all physical forces. But this does not have to be the only possible point of view. One can view the pure Affine description from axiomatic point of view only as a way obtaining the equations of motion without any geometrical considerations what so ever.

We begin by postulating that Total Matter $\left(\mathbf{M}^{\mathrm{T}}\right)$ is a set of functions associated with a four-dimensional continuum and thus is characterized by means of parameters $x^{i}(i=0,1,2,3)$-the coordinates. The description of Matter should not depend on the system coordinates and thus it should have some group property with respect to transition from one system of coordinate to another.

The functions of Matter $\left(\mathbf{M}^{\mathrm{T}}\right)$ also must have "potential" $-\boldsymbol{\Gamma}$, that is to say that $\mathbf{M}^{\mathrm{T}}$ should be expressed thru a set of functions (we call "potential") and its first partial derivatives:

$$
\mathbf{M}^{\mathrm{T}}=\mathbf{M}^{\mathrm{T}}\left(\frac{\partial \boldsymbol{\Gamma}}{\partial x_{i}}, \boldsymbol{\Gamma}\right) .
$$

An example of such pair $\left(\mathbf{M}^{\mathrm{T}}, \boldsymbol{\Gamma}\right)$ could be the Eddington's set of affine connections and a generalized curvature tensor built on them:

$$
\mathbf{M}^{\mathrm{T}} \equiv M_{j k l}^{\mathrm{Ti}} ; \quad \boldsymbol{\Gamma} \equiv \Gamma_{j k}^{i} ; M_{j k l}^{T i}=\Gamma_{j l, k}^{i}-\Gamma_{j k, l}^{i}+\Gamma_{k m}^{i} \Gamma_{j l}^{m}-\Gamma_{l m}^{i} \Gamma_{j k}^{m}
$$

where $\Gamma \equiv \Gamma_{j k}^{i}$ in general are non-symmetrical in low indeces. The above expression also could be written thru symmetric part of $\Gamma$ connections $\stackrel{s}{\Gamma} \equiv \stackrel{\Gamma}{j}_{j k}^{i}=\frac{1}{2}\left(\Gamma_{j k}^{i}+\Gamma_{k j}^{i}\right)$ - which $\underset{a}{i} \underset{i}{i}$ affine connections-and thru anti-symmetric parts of connections $\stackrel{a}{\Gamma} \equiv \stackrel{s}{\Gamma}_{j k}^{i}=\frac{1}{2}\left(\Gamma_{j k}^{i}-\Gamma_{k j}^{i}\right)$-which are tensors-in the following manner:





where vertical bar $(\mid)$ indicates covariant derivative of the tensor $\stackrel{a}{\Gamma}$ with re- 
spect to symmetrical part of $\Gamma$.

The above expression (8) of $\mathbf{M}^{\mathrm{T}}$ thru $\boldsymbol{\Gamma}$ historically is derived from geometrical considerations. And since we are not bound by any geometrical considerations and because $\stackrel{a}{\Gamma}_{j k}^{i} \equiv \Gamma_{j k}^{i}-\Gamma_{k j}^{i}$ is a tensor, we can consider (keeping the same frame-work: linear with respect to $\partial_{i} \boldsymbol{\Gamma}$, quadratic with respect $\boldsymbol{\Gamma}$ and anti-symmetrical with respect to two low indeces " $k-l$ " of the tensor $\mathbf{M}_{j k l}^{\mathrm{T} i}$ ) more generalized expression thru new set of functions, derived from $\Gamma$-vector $\stackrel{a}{\Gamma_{k}}$ and anti-symmetric traceless ("atl”) in “ $j-k$ ” indeces tensor $\stackrel{a t l^{i}}{\Gamma_{j k}}$ defined below:

$$
\begin{aligned}
& \stackrel{a}{\Gamma} k=\stackrel{a^{i}}{\Gamma_{i k}} ; \quad \stackrel{a t l}{\Gamma}_{j k}^{i}=\stackrel{a}{\Gamma}_{j k}^{i}-\frac{1}{3}\left(\delta_{j}^{i} \stackrel{a}{\Gamma} k-\delta_{k}^{i} \stackrel{a}{\Gamma} k\right) ; \quad \stackrel{a t l^{i}}{\Gamma_{i k}}=0 \\
& \left(M^{\mathrm{T}}\right)_{j k l}^{i}=\stackrel{s}{\Gamma}_{j l, k}^{i}-\stackrel{s}{\Gamma}_{j k, l}^{i}+\stackrel{s}{\Gamma}_{m k}^{i} \stackrel{s}{\Gamma}_{j l}^{m}-\stackrel{s}{\Gamma}_{m l}^{i} \stackrel{s}{\Gamma}_{j k}^{m}
\end{aligned}
$$



$$
\begin{aligned}
& +\mu_{5}\left(\delta_{k}^{i} \stackrel{a}{\Gamma}_{j \mid l}-\delta_{l}^{i} \stackrel{a}{\Gamma}_{j \mid k}\right)+\mu_{6}\left(\delta_{k}^{i} \stackrel{a}{\Gamma} l \mid j-\delta_{l}^{i} \stackrel{a}{\Gamma}_{k \mid j}\right)+\mu_{7} \delta_{j}^{i}\left(\stackrel{a}{\Gamma}_{k \mid l}-\stackrel{a}{\Gamma}_{l \mid k}\right)
\end{aligned}
$$

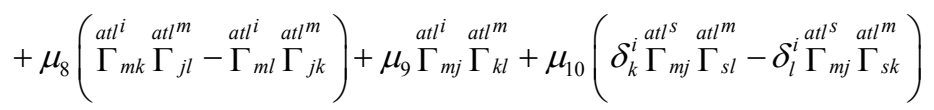

$$
\begin{aligned}
& +\mu_{11}\left(\delta_{k}^{i} \Gamma_{m}^{a} \stackrel{a t l}{\Gamma}_{j l}^{m}-\delta_{l}^{i} \stackrel{a}{\Gamma}_{m} \stackrel{a t l^{m}}{\Gamma_{j k}}\right)+\mu_{12} \delta_{j}^{i} \stackrel{a}{\Gamma}_{m}^{a t l^{m}} \Gamma_{k l}+\mu_{13} \stackrel{a}{\Gamma_{j}}{\stackrel{a t l^{i}}{\Gamma}}_{k l} \\
& +\mu_{14}\left(\stackrel{a}{\Gamma} \stackrel{a t l}{\Gamma}_{j l}^{i}-\stackrel{a}{\Gamma} \stackrel{a t l^{i}}{\Gamma} j k\right)+\mu_{15}\left(\delta_{k}^{i} \stackrel{a}{\Gamma} \stackrel{a}{\Gamma}_{l}-\delta_{l}^{i} \stackrel{a}{\Gamma} \stackrel{a}{\Gamma}_{k}\right)
\end{aligned}
$$

where $\mu_{1}$ thru $\mu_{15}$ are constants. The choice of these constants is not a strait forward procedure. It will eventually be defined by a form of Lagrangian that should reflect the physical reality. We will discuss the value of the $\mu$-constants mostly in the Section 8-Electromagnetic and Other Vector Fields.

The equation of motions of the Total Matter is obtained by means of variation of the Lagrangian density $L^{\sqrt{ }}$ that is a function of tensor $\mathbf{M}^{\mathrm{T}}$ only and hence is a function of $\Gamma$ and its first partial derivatives only.

$$
S=\int L^{\sqrt{ }}\left(\mathbf{M}^{\mathrm{T}}\right) \mathrm{d}^{4} x=\int L^{\sqrt{ }}\left(\partial_{i} \boldsymbol{\Gamma}, \boldsymbol{\Gamma}\right) \mathrm{d}^{4} x ; \text { equations of motions }: \frac{\delta S}{\delta \boldsymbol{\Gamma}}=0
$$

The Eddington's original idea was to equate $L^{\sqrt{ }}$ with determinate of symmetric tensor obtain by contracting tentor $\mathbf{M}^{\mathrm{T}}$.

$$
\mathbf{M}_{(R i)}^{\mathrm{T}} \equiv\left(M_{(R i)}^{\mathrm{T}}\right)_{j l}=\frac{1}{2}\left[\left(M^{\mathrm{T}}\right)_{j k l}^{k}+\left(M^{\mathrm{T}}\right)_{l k j}^{k}\right] ; L^{\nwarrow}=\sqrt{\left|\operatorname{det}\left(M_{(R i)}^{\mathrm{T}}\right)\right|}
$$

More complex Lagrangians could be formed by contracting $n$-power product of the Matter tensor using the inverse tensor to the tensor $\mathbf{M}_{(R i)}$ (as long as its determinant is not zero). This symbolically could be written as:

$$
L^{\sqrt{ }}=\sum_{n=1,2 \ldots s=\text { allinv }} \lambda_{n s}\left(\mathbf{M}^{\mathrm{T}}\right)^{n}\left(\mathbf{M}_{(R i)}^{\mathrm{T}}\right)^{-n} \sqrt{\left|\operatorname{det}\left(\mathbf{M}_{(R i)}^{\mathrm{T}}\right)\right|}
$$

where index " $s$ " varies over all possible invariants taken for each " $n$-power product" and $\lambda_{n s}$ are some constants corresponding to these invariants. 
It is not difficult to see that maximum power " $n$ " includes all the terms with the lower powers. For example, if we choose $n=4$, we automatically included Lagrangians with the $n=1,2$ and 3 .

$$
\begin{aligned}
L^{\sqrt{ }} & =\sum_{s=\text { allinv }} \lambda_{s}^{(4)}\left(\mathbf{M}^{\mathrm{T}}\right)^{4}\left(\mathbf{M}_{(R i)}^{\mathrm{T}}\right)^{-4} \sqrt{\operatorname{det}\left(\mathbf{M}_{(R i)}^{\mathrm{T}}\right) \mid} \\
& \equiv \sum_{n=1,2,3,4 s=\text { allinv }} \lambda_{n s}\left(\mathbf{M}^{\mathrm{T}}\right)^{n}\left(\mathbf{M}_{(R i)}^{\mathrm{T}}\right)^{-n} \sqrt{\operatorname{det}\left(\mathbf{M}_{(R i)}^{\mathrm{T}}\right) \mid}
\end{aligned}
$$

The quadratic $(n=2)$ to $\mathbf{M}^{\mathrm{T}}$ Lagrangians were extensively studied previously, but eventually dropped without much progress.

Among the reasons for that was an assumption that, as it is in GR, the gravitation is described by metric only. In this paper (as it was mentioned earlier) we assume that gravitation is describe by a pair metric tensor and vector field which value is much (much) larger than any other field.

There are two important questions here that need be answered: a) what the values of " $n$ " (including the maximum value) should be? and b) what are the value of the all $\lambda$-constants?

Regarding the $\lambda_{n s}$-constants, the procedure of determining their values is the following. We will consider a "small system" (say our Solar system as compared to our Galaxy) of Matter and will derive its Lagrangian from the Lagrangian of the Total-Matter (12). During this transitioning procedure we will have some "unwanted" terms, which-as we will see later in the Section 6-Lagrangianwill be of the same form for any value " $n$ " (no matter how large " $n$ " is) except for the values of $\lambda_{n s}$-constants. And so we will require that the $\lambda_{n s}$-constants were chosen in a such way that all the "unwanted" terms vanished.

The quadratic Lagrangian does not provide enough invariants (or enough $\lambda$ s) to cancel all "unwanted" terms so we ought to increase $n$ to 3 . And if that is not enough we will increase " $n$ " to 4 and so. With increase of " $n$ " the number of invariants increases rather rapidly and it would be reasonable to assume that with $n=4$ (or 5) all "unwanted" terms could be canceled. This however brings another problem: what to do if we have too many $\lambda$-s? Let say that $n=4$ is not enough, but $n=5$ has too many $\lambda$-s. The obvious solution here is to exclude lowest " $n$ " numbers. That is if $n=2,3,4,5$ has to many $\lambda$-s use only $n=3,4,5 \cdots$ and so on. Of course we are not guaranteed that $n=3,4,5$ (or $n=4,5$ ) would just perfectly enough and we would have to come up with other strategy to eliminate the extras $\lambda$-s.

Jumping ahead we can say that the simple estimation of number invariants and the number of unwanted terms suggests that the good value for $n$ is $n=4$, so the Lagrangian has this form:

$$
L^{\sqrt{ }}=\sum_{s=\text { allinv }} \lambda_{s}^{(4)}\left(\mathbf{M}^{\mathrm{T}}\right)^{4}\left(\mathbf{M}_{(R i)}^{\mathrm{T}}\right)^{-4} \sqrt{\left|\operatorname{det}\left(\mathbf{M}_{(R i)}^{\mathrm{T}}\right)\right|} .
$$

The $n=4$ is also supported by the fact that the Determinant of $\left(\mathbf{M}_{(R i)}^{\mathrm{T}}\right)$ could be written as the 4 th power product: 


$$
\operatorname{det}\left(\left(\mathbf{M}_{(R i)}^{\mathrm{T}}\right)\right)=\sum\left(\mathbf{M}_{(R i)}^{\mathrm{T}}\right)^{4} \Delta^{2}
$$

where $\Delta$ is a Levi-Civita fully antisymmetric (all indeces up) "almost tensor" $\left(\Delta \equiv \Delta^{i j k l}=(-1)^{p}\right.$, where $p$ ia a number of permutations going from "1234" to “ijkl”).

In the end this question cannot be answered without doing actual calculations and/or employing some other principals for choosing the general form of the Lagrangian.

So in this section we would like simply to mention some of the interesting options that we have based on solely "beauty" form.

Among the Lagrangians of the 4th power we need to mention a particular one:

$$
L^{\sqrt{ }}=\left[\sum_{s=\text { inv }} \lambda_{s}\left(\mathbf{M}^{\mathrm{T}}\right)^{4} \Delta_{M}^{2}\right] \sqrt{\left|\operatorname{det}\left(\mathbf{M}_{(R i)}^{\mathrm{T}}\right)\right|}
$$

where $\boldsymbol{\Delta}_{M}$ is a fully antisymmetric Levi-Civita (all indeces up) tensor based on the Ricci contraction of the Total-Matter tensor $\left(\Delta \equiv \Delta^{i j k l} / \sqrt{\left|\operatorname{det}\left(\mathbf{M}_{(R i)}^{\mathrm{T}}\right)\right|}\right.$.

In this case the Lagrangian density can be also written as:

$$
L^{\sqrt{ }}=\sum_{s=\text { inv }} \lambda_{s}\left(\mathbf{M}^{\mathrm{T}}\right)^{4} \Delta_{r}^{2} \sqrt{\frac{|\operatorname{det}(\mathbf{r})|}{\left|\operatorname{det}\left(\mathbf{M}_{(R i)}^{\mathrm{T}}\right)\right|}} \sqrt{\operatorname{det}(\mathbf{r}) \mid}
$$

where $\boldsymbol{\Delta}_{r}$ is a Levi-Civita tensor (all indeces up) constructed using metric tensor $r_{i j}$, what ever a definition of $r_{i j}$ as a function of $\Gamma$ is. This Lagrangian can be also written as:

$$
L^{\sqrt{ }}=\sum_{n=2,3,4 s=\text { allinv }} \lambda_{n s}\left(\mathbf{M}^{\mathrm{T}}\right)^{4} \mathbf{r}^{-4} \sqrt{\frac{|\operatorname{det}(\mathbf{r})|}{\left|\operatorname{det}\left(\mathbf{M}_{(R i)}^{\mathrm{T}}\right)\right|}} \sqrt{|\operatorname{det}(\mathbf{r})|} .
$$

The beauty of the above Lagrangian is that it does have a rather attractive and esthetically appealing properties: a) it is minimal dependence on the tensor $\left.\mathbf{M}_{(R i)}^{\mathrm{T}}, \mathrm{b}\right)$ its elegance and simplicity. Mathematically it almost equate tensor $\mathbf{M}_{(R i)}^{\mathrm{T}}$ with metric tensor $\mathbf{r}$ without actually equating it.

The above Lagrangian can be generalized to deliver much more parameters $\lambda_{s}$. It utilizes not two but multiple number of "two-s" Levi-Civita tensors built on $\mathbf{M}_{(R i)}^{\mathrm{T}}$

$$
L^{\sqrt{ }}=\sum_{n=1,2 \ldots} \sum_{s} \lambda_{n s}\left(\mathbf{M}^{\mathrm{T}}\right)^{4 n} \boldsymbol{\Delta}_{M}^{2 n} \sqrt{\operatorname{det}\left(\mathbf{M}_{(R i)}^{\mathrm{T}}\right) \mid}
$$

which as before-expr. (17) — could be switched to the metric based invariants:

$$
L^{\mathcal{V}}=\sum_{n} \sum_{s}\left(\lambda_{s} \mathbf{M}^{\mathrm{T}}\right)^{4 n} \Delta_{r}^{2 n}\left[\frac{\operatorname{det}(\mathbf{r})}{\operatorname{det}\left(\mathbf{M}_{(R i)}^{\mathrm{T}}\right)}\right]^{n-\frac{1}{2}} \sqrt{|\operatorname{det}(\mathbf{r})|} .
$$

Everywhere above we assumed that $\operatorname{det}\left(\mathbf{M}_{(R i)}^{\mathrm{T}}\right) \neq 0$, thus sub-selecting $\mathbf{M}_{(R i)}^{\mathrm{T}}$ from the tensor of Total-Matter $\mathbf{M}^{\mathrm{T}}$. There is however a possibility of Lagrangian which avoid this non-zero requirement, and thus putting all terms of the 
tensor of Total-Matter on equal footing.

$$
\begin{aligned}
& L^{\sqrt{ }}=\sqrt{\sum_{s} \lambda_{s}^{(1)}\left(\mathbf{M}^{\mathrm{T}}\right)^{4} \boldsymbol{\Delta}^{2}}-\sqrt{\sum_{s} \lambda_{s}^{(2)}\left(\mathbf{M}^{\mathrm{T}}\right)^{4} \boldsymbol{\Delta}^{2}} \\
& \text { where } \boldsymbol{\Delta}=(-1)^{p} \quad p=\text { permut'n number }
\end{aligned}
$$

where $\lambda_{s}^{(1)}$ and $\lambda_{s}^{(2)}$ are two sets of $\lambda$-constants-one for each "square root". For example the set $\lambda_{s}^{(2)}$ could be chosen in such manner that second square root is a $\sqrt{ \pm \operatorname{det}\left(\mathbf{M}_{(R i)}^{\mathrm{T}}\right)}$.

With our assumption that Gravitational field accumulates to a very large value, it can be shown that it is in effect equivalent to the condition that $\mathbf{M}_{(R i)}^{\mathrm{T}}$ has a very large value and much bigger than any other field$\mathbf{M}^{\mathrm{T}}-\mathbf{M}_{(R i)}^{\mathrm{T}} \equiv\left(\mathbf{M}^{\mathrm{T}}\right)_{j k l}^{i}-1 / 3\left[\delta_{k}^{i}\left(\mathbf{M}_{(R i)}^{\mathrm{T}}\right)_{j l}-\delta_{l}^{i}\left(\mathbf{M}_{(R i)}^{\mathrm{T}}\right)_{j k}\right] \ll \mathbf{M}_{(R i)}^{\mathrm{T}}$-and thus the expression above could be approximately written in a form (14):

$$
\begin{aligned}
L^{\sqrt{ }} & =\sqrt{\sum_{s} \lambda_{s}^{(1)}\left(\mathbf{M}^{\mathrm{T}}\right)^{4} \boldsymbol{\Delta}^{2}}-\sqrt{\sum_{s} \lambda_{s}^{(2)}\left(\mathbf{M}^{\mathrm{T}}\right)^{4} \boldsymbol{\Delta}^{2}} \\
& =\sqrt{\operatorname{det}\left(\mathbf{M}_{(R i)}^{\mathrm{T}}\right)}\left[\sqrt{\sum_{s} \lambda_{s}^{(1)}\left(\mathbf{M}^{\mathrm{T}}\right)^{4} \boldsymbol{\Delta}_{M}^{2}}-\sqrt{\sum_{s=\text { inv }} \lambda_{s}^{(2)}\left(\mathbf{M}^{\mathrm{T}}\right)^{4} \boldsymbol{\Delta}_{M}^{2}}\right] \\
& =\frac{\sqrt{\operatorname{det}\left(\mathbf{M}_{(R i)}^{\mathrm{T}}\right)}\left[\sum_{s} \lambda_{s}^{(1)}\left(\mathbf{M}^{\mathrm{T}}\right)^{4} \boldsymbol{\Delta}_{M}^{2}-\sum_{s=\text { inv }} \lambda_{s}^{(2)}\left(\mathbf{M}^{\mathrm{T}}\right)^{4} \boldsymbol{\Delta}_{M}^{2}\right]}{\sqrt{\sum_{s} \lambda_{s}^{(1)}\left(\mathbf{M}^{\mathrm{T}}\right)^{4} \boldsymbol{\Delta}_{M}^{2}}+\sqrt{\sum_{s} \lambda_{s}^{(2)}\left(\mathbf{M}^{\mathrm{T}}\right)^{4} \boldsymbol{\Delta}_{M}^{2}}} \\
& =\frac{\sqrt{\operatorname{det}\left(\mathbf{M}_{(R i)}^{\mathrm{T}}\right)}\left[\sum_{s}\left(\lambda_{s}^{(1)}-\lambda_{s}^{(2)}\right)\left(\mathbf{M}^{\mathrm{T}}\right)^{4} \boldsymbol{\Delta}_{M}^{2}\right]}{\sqrt{\lambda_{0}^{(1)}+\sum_{n=2,3,4}^{s=\text { inv }} \lambda_{s}^{(1)}\left(\tilde{\mathbf{M}}^{\mathrm{T}} \mathbf{M}_{(R i)}^{\mathrm{T}}\right)^{-n}}+\sqrt{\lambda_{0}^{(2)}+\sum_{n=2,3,4}^{s=\text { inv }} \lambda_{s}^{(2)}\left(\tilde{\mathbf{M}}^{\mathrm{T}}\right)^{4}\left(\mathbf{M}_{(R i)}^{\mathrm{T}}\right)^{-4}}} \\
& \approx \frac{\sqrt{\operatorname{det}\left(\mathbf{M}_{(R i)}^{\mathrm{T}}\right)}\left[\sum_{s}\left(\lambda_{s}^{(1)}-\lambda_{s}^{(2)}\right)\left(\mathbf{M}^{\mathrm{T}}\right)^{4} \boldsymbol{\Delta}_{M}^{2}\right]}{\sqrt{\lambda_{0}^{(1)}+\sqrt{\lambda_{0}^{(1)}}}[}
\end{aligned}
$$

where $\Delta_{M}$ is a Levi-Civita tensor based on symmetrical tensor

$$
\mathbf{M}_{(R i)}^{\mathrm{T}}\left(\boldsymbol{\Delta}_{M} \equiv \boldsymbol{\Delta} \frac{1}{\sqrt{\operatorname{det}\left(M_{(R i)}^{\mathrm{T}}\right)}}\right) \text {. }
$$

The above Lagrangian as we just showed will deliver the same result as the (14) for a "small system" of matter for which $\mathbf{R}_{(R i)}$ locally is very large. However, it will give significantly different form for the Cosmological problem. In it we must assume that the "averaged" value of the Gravitational field is defined by all Matter and thus the assumption that $\mathbf{M}^{\mathrm{T}} \approx \mathbf{M}_{(R i)}^{\mathrm{T}}$ may not hold.

\section{Tensor Potential and Metric Definition}

Up to this point we defined the Matter and the equations of motion by means of only $\Gamma$ functions and without any reference to the space metric. The space metric is of course needed (at lease to calculate the distances between points). For any given metric $\mathbf{r}$ one can write the expression for $\Gamma$ thru symbols Christof- 
fel in the form (written symbolically) $\boldsymbol{\Gamma}=\gamma+\mathbf{P}^{\mathrm{T}}$ :

$$
\Gamma_{j k}^{i}=\gamma_{j k}^{i}+\left(P^{\mathrm{T}}\right)_{j k}^{i} ; \quad \gamma_{j k}^{i}=\frac{1}{2} r^{i s}\left(r_{s j, k}+r_{s k, j}-r_{j k, s}\right)
$$

where $\mathbf{P}^{\mathrm{T}}$ is a 3-index (1-up and 2-down) tensor, which we label a "TensorPotential" and bold upper index ${ }^{\mathrm{T}}$ refers to the fact that it's a Tensor-Potential of all Matter.

By choosing different metric tensors $\mathbf{r}$ we will modify the Tensor-Potential $\mathbf{P}^{\mathrm{T}}$. Our goal here to eliminate some of the component of the tensor $\mathbf{P}^{\mathrm{T}}$ and by doing so to replace some of the $\mathbf{P}^{\mathrm{T}}$ with description by metric $\mathbf{r}$. This of course must be done in tensorial manner-certain components of the tensor $\mathbf{P}^{\mathrm{T}}$ should vanish.

From (23) it's clear that it will lead to a set of first order partial equations with respect to the metric tensor $\mathbf{r}$. The question here is how many component-or independent equations on $r_{i j}$-could we eliminate? There are 10 functions in a metric tensor $\mathbf{r}$ and it seems logical that the number first order partial derivative equations should be 20. But here comes a consideration that the equations for $\mathbf{r}$ are written in covariant (tensorial) form. This means that they include 4 arbitrary functions of transformation from one system coordinate to another. In order to account for that the logic here should be modified as following. There are 6 independent functions in a metric tensor $\mathbf{r}$. Hence there should be twice that many (12) equations of the first order of partial equations for the $\mathbf{r}$. But since they are written in covariant way (in any system coordinates with 4 arbitrary functions) then the number of equation should increased by 4 to 16 .

In order to identify those 16 functions we will decompose the tensor $P_{j k}^{\mathrm{Ti}}$ on some its components. After lowering upper index with metric tensor $\mathbf{r}$ (to be the first low index) $-P_{i j k}^{\mathrm{T}}=\left(P^{\mathrm{T}}\right)_{j k}^{m} r_{m i}$-we can split the tensor $P_{i j k}^{\mathrm{T}}$ on 4 vectors $G_{i}, B_{i}, E_{i}, D_{i}$ and 3 traceless 3 -index tensors-fully symmetrical tensor $N_{i j k}$ and two torsion type (asymmetric in indeces $j k$ ) tensors $K_{i j k}\left(K_{i j k}=-K_{i k j}\right)$ and $C_{i j k}\left(C_{i j k}=-C_{i k j}\right)$. If decomposition of $\stackrel{a}{\mathbf{P}}^{\mathrm{T}}$ is unique, it is not so for the symmetrical in low indeces part of $\Gamma_{j k}^{i}-\left(\stackrel{s}{\mathbf{P}}^{\mathrm{T}}\right)$. Its generic decomposition form can be written as:

$$
\begin{aligned}
& P_{i j k}^{\mathrm{T}} \equiv P_{j k}^{\mathrm{T} m} r_{m i}=\stackrel{s \mathrm{~T}}{P_{i j k}}+\stackrel{a \mathrm{~T}}{P} \stackrel{s \mathrm{~T}}{P_{i j k}} \stackrel{P}{i j k}^{\mathrm{T}}=\stackrel{s \mathrm{~T}}{P_{i k j}} ; \stackrel{a \mathrm{~T}}{P_{i j k}}=-\stackrel{a \mathrm{~T}}{P_{i k j}} \\
& \stackrel{s \mathrm{~T}}{P_{i j k}}=\stackrel{s-t t l}{P}_{i j k}^{\mathrm{T}}+G_{j} r_{i k}+G_{k} r_{i j}+\pi_{1} G_{i} r_{j k}+\pi_{2} \frac{1}{G^{2}} G_{i} G_{j} G_{k}+B_{j} r_{i k}+B_{k} r_{i j}+\pi B_{i} r_{j k} \\
& \text { where } \stackrel{s-t l}{P} \stackrel{\mathrm{T}}{i j k}^{\mathrm{T}} \text { traceless : } \stackrel{s-t l{ }_{i j k}^{\mathrm{T}}}{P} r^{i j}=0 ; \stackrel{s-t l l_{i j k}^{\mathrm{T}}}{r^{j k}}=0
\end{aligned}
$$

where for definition of vectors $\mathbf{G}$ and $\mathbf{B}$ we get these two equations:

$$
\begin{aligned}
& \stackrel{s}{P}_{k}^{\mathrm{T}(1)} \equiv \stackrel{s}{P}_{i j k}^{\mathrm{T}(1)} r^{i j}=\left(5+\pi_{1}+\pi_{2}\right) G_{k}+(5+\pi) B_{k} \\
& \stackrel{s}{P}(2)_{i} \equiv \stackrel{s}{P}_{i j k}^{\mathrm{T}(2)} r^{j k}=\left(2+4 \pi_{1}+\pi_{2}\right) G_{i}+(2+4 \pi) B_{i}
\end{aligned}
$$


The above decomposition is reversible if the determinant is not zero in which case $G_{i}$ and $B_{i}$ are given by this expression thru symmetric part of the Tensor-Potential $\stackrel{s}{P}^{\mathrm{T}}$ :

$$
\begin{gathered}
18\left(\pi-\pi_{1}\right)+3 \pi_{2}(\pi-1) \neq 0 \\
G_{i}=\frac{(2+4 \pi) \stackrel{s}{P}_{n i m}^{\mathrm{T}} r^{m n}-(5+\pi) \stackrel{s}{P}_{\text {imn }}^{\mathrm{T}} r^{m n}}{18\left(\pi-\pi_{1}\right)+3 \pi_{2}(\pi-1)} ; \\
B_{i}=\frac{-\left(2+4 \pi_{1}+\pi_{2}\right) \stackrel{s}{P}_{\text {min }}^{\mathrm{T}} r^{m n}+\left(5+\pi_{1}+\pi_{2}\right) \stackrel{s}{P}^{s}{ }_{i m n}^{\mathrm{T}} r^{m n}}{18\left(\pi-\pi_{1}\right)+3 \pi_{2}(\pi-1)}
\end{gathered}
$$

Most of this paper we will assume that $\pi=0, \pi_{2}=-3\left(1+\pi_{1}\right)$ and $\pi_{1}$ is either root of this this quadratic equation: $\pi_{1}^{2}-3 \pi_{1}-3=0$, so $\pi_{1}=(3+\sqrt{21}) / 2 \approx 3.8$ or $\pi_{1}=(3-\sqrt{21}) / 2 \approx-0.8$.

The fully symmetric tensor $N_{i j k}$ and torsion type tensor $K_{i j k}$ expressed thru symmetric and traceless (“s- $\mathrm{tl}$ ”) $\stackrel{s-t t \mathrm{~T}^{\mathrm{T}}}{P_{i j k}}$ as:

$$
\begin{aligned}
& N_{i j k}=\frac{1}{3}\left(\stackrel{s-t l}{P}_{i j k}^{\mathrm{T}}+\stackrel{s-t l}{P}_{i j k}^{\mathrm{T}}+\stackrel{s-t l}{P}_{i j k}^{\mathrm{T}}\right) N_{i j k} r^{j k}=0 \\
& K_{i j k}=-K_{i k j}=\frac{1}{3}\left(\begin{array}{l}
s-t l \\
P \\
P i k
\end{array} \stackrel{s-t l}{P}_{k i j}^{\mathrm{T}}\right) ; K_{i j k} r^{i j}=0 ; K_{i j k} \Delta^{i j k l}=0
\end{aligned}
$$

For the decomposition of anti-symmetric part of the Tensor-Potential $\stackrel{a^{T}}{P_{i j k}}$ on two vectors $D_{i}$ and $E_{i}$ (which is actually fully antisymmetric tensor) and torsion type tensor $C_{i j k}=-C_{i k j}$ we have":

$$
\begin{aligned}
& \stackrel{a}{P}_{i j k}^{\mathrm{T}}=C_{i j k}+\left(D_{k} r_{i j}-D_{j} r_{i k}\right)+E_{n} r^{m n} \Delta_{i j k m} \sqrt{\left|\operatorname{det}\left(r_{i j}\right)\right|} \\
& C_{i j k}=-C_{i k j} ; C_{i j k} r^{i j}=0 ; \quad C_{i j k} \Delta^{i j k l}=0 \text { where } \Delta^{i j k l}=(-1)^{p} \quad p \text {-permutation } \\
& D_{i}=\frac{1}{3} \stackrel{s-t l}{P}^{\mathrm{T}} r_{i j k}^{i j} \text { and } E_{i}=-\frac{1}{6} r_{i m} \stackrel{a}{P}_{n s t}^{\mathrm{T}} \Delta^{m n s t} \frac{1}{\sqrt{\left|\operatorname{det}\left(r_{p q}\right)\right|}}
\end{aligned}
$$

The important fact here is that the vectors $\mathbf{G}, \mathbf{B}, \mathbf{D}, \mathbf{E}$ as well as the tensors $\mathbf{N}, \mathbf{K}, \mathbf{C}$ are fully defined by the tensor potential $\mathbf{P}^{\mathrm{T}}$ or its symmetric in and anti-symmetrical parts $\stackrel{s}{\mathbf{P}}^{\mathrm{T}}$ and $\stackrel{a}{\mathbf{P}}^{\mathrm{T}}$.

Because of their "traceless" properties the 3 -index tensors $\mathbf{N}, \mathbf{K}$ and $\mathbf{C}$ consists of 16 (exactly as we need) independent functions. We will use this fact and will define metric tensor $\mathbf{r}$ in a such way that one of these 3-index tensors is eliminated.

There are two ways of doing it: a) to set the tensor $\mathbf{N}$ to zero, b) set $\mathbf{K}$ to be proportional to the tensor $\mathbf{C}\left(\mathbf{K}=\mu_{0} \mathbf{C}\right)$. If $\mu_{0}$ is zero we effectively eliminate the tensor $\mathbf{K}$. In this paper we choose the case " $\mathrm{b}$ " with non-zero $\mu_{0}$. The reason for that is that the case " $b$ " leads to linear system of equations with respect to metric derivatives $\left(\mathbf{r}^{\prime}\right)$, which is not true for the case " $a$ " due to the presence of the $G_{i} G_{j} G_{k}$ term in expr. (23). This leads to 16 linear first order 
differential equations for the metric tensor $\mathbf{r}$ thru affine connections $\Gamma$.

Thus we replaced a part (16 components) of the tensor potential $\mathbf{P}^{\mathrm{T}}$ with 10 components of metric tensor, making the latter a dynamic variable along with remaining 48 components of the tensor $\mathbf{P}^{\mathrm{T}}: 16$ for each tensor $\mathbf{N}$ and $\mathbf{C}$ and 16 for four vectors $\mathbf{G}, \mathbf{B}, \mathbf{D}, \mathbf{E}$.

For the sake of being "complete" we need here to mention that the equations for metric should also include a term "conjugate" to the tensor $\mathbf{C}-\boldsymbol{\Delta}_{r} \mathbf{C}$, which will not change significantly their form (its linearity, etc.):

$$
K_{i j k}^{P}=\mu_{0} C_{i j k}+\hat{\mu}_{0} \frac{1}{\sqrt{\left|\operatorname{det}\left(r_{i j}\right)\right|}} r_{i m} r_{j n} \Delta^{m n p q} C_{k p q} \text { or symbolically } \mathbf{K}=\mu_{0} \mathbf{C}+\hat{\mu}_{0}^{*} \mathbf{C} \text {. }
$$

After defining metric $\mathbf{r}$, we can transfer Eddington pure affine formulation into a more physically familiar metric-Matter formalism.

$$
S=\int \mathrm{d}^{4} x \sum_{n=1, \cdots} L^{(n)} \text { with } L^{(n)}=\sum_{s=\text { inv }} \lambda_{s}^{(n)}\left[\mathbf{M}^{\mathrm{T}}\left(\mathbf{P}^{\mathrm{T}}\right)\right]^{n}\left[\mathbf{M}_{(R i)}^{\mathrm{T}}\left(\mathbf{P}^{\mathrm{T}}\right)\right]^{-n} \sqrt{\mid \operatorname{det}\left[\mathbf{M}_{(R i)}^{\mathrm{T}}\left(\mathbf{P}^{\mathrm{T}}\right)\right]}
$$

where the tensor of Total-Matter $\mathbf{M}^{\mathrm{T}}$ is now be written thru metric $\mathbf{r}$, Riemann curvature tensor $\mathbf{R}$ vectors $\mathbf{G}, \mathbf{B}, \mathbf{D}, \mathbf{E}$ and 3-index tensors $\mathbf{N}$, C. And the equations of motions for each variable is obtained by variation of Lagrangian (30) above by these variables.

We now attach some physical meaning to the fields. Or to be more accurate we try to identify which of 4 vector fields is the Gravitational field. The main property of gravitational field-and this is our main postulate-is that it is associated with every mass particle (proton, etc.) and does get accumulated and reaches very large value, which is much bigger that any other field. Thus in the vacuum the Total-Matter consists only of that field. Since in general, the tensor of Total-Matter has quadratic form, it clear that the Gravitational field in vacuum should be represented by the square of one of the vector fields.

Between 4 vectors $\mathbf{G}, \mathbf{B}, \mathbf{D}$ and $\mathbf{E}$ the last three ones should be rejected. The reason for that is the requirement-see Equation (12)—that $\operatorname{det}\left(\mathbf{M}_{(R i)}^{\mathrm{T}}\right) \neq 0$. But in a case of constant field and flat space-which is always the case in the infinitely small area-the $\operatorname{det}\left(\mathbf{M}_{(R i)}^{\mathrm{T}}\right)$ for either vector field-E, $\mathbf{D}$ or $\mathbf{D}-$ is zero.

For example, calculations for the quadratic terms of the vector field $\mathbf{D}$ the Total-Matter-see expr. (9)-has this form: $\mu_{15}\left(D_{j} D_{l} r_{i k}-D_{j} D_{k} r_{i l}\right)$. The tensor of Ricci for the Total-Matter for only constant vector $\mathbf{D}$ has this form:

$$
\begin{aligned}
& \mu_{15}\left(D_{j} D_{l} r_{i k}-D_{j} D_{k} r_{i l}\right)=>\left(M_{(R i)}^{\mathrm{T}(D)}\right)_{j l} \equiv M_{i j k l}^{\mathrm{T}(D)} r^{i k}=3 \mu_{15} D_{j} D_{l} ; \\
& \text { and } \operatorname{det}\left[\left(M_{(R i)}^{\mathrm{T}(D)}\right)_{j l}\right]=0 .
\end{aligned}
$$

Similarly, is true for the vector $\mathbf{E}$. The tensor of Total-Matter for only $\mathbf{E}$ field (its quadratic terms) has this expression:

$$
\begin{aligned}
& M_{i j k l}^{\mathrm{T}(E)}=\left(\mu_{7}+\mu_{8}+\mu_{9}\right)\left[E^{2}\left(r_{i k} r_{j l}-r_{i l} r_{j k}\right)-\left(r_{i k} E_{j} E_{l}-r_{i l} E_{j} E_{k}\right)-\left(r_{j l} E_{i} E_{k}-r_{j k} E_{i} E_{l}\right)\right] \\
& \left(M_{(R i)}^{\mathrm{T}(E)}\right)_{j l}=\left(\mu_{7}+\mu_{8}+\mu_{9}\right)\left[(E)^{2} r_{j l}-E_{j} E_{l}\right] \Rightarrow \operatorname{det}\left[\left(M_{(R i)}^{\mathrm{T}}\right)_{j l}\right]=0
\end{aligned}
$$


Calculations quadratic terms for the vector field B produce the following result for the tensor $\left(M_{(R i)}^{\mathrm{T}(B)}\right)_{j l}$ :

$$
\begin{aligned}
\stackrel{s}{P} \mathrm{~T}(B)^{\mathrm{T}(B) k} & B_{j} r_{i k}+B_{k} r_{i j}+\pi B_{i} r_{j k} ; M_{i j k l}^{\mathrm{T}(B)} \approx\left(P^{\mathrm{T}(B)}\right)_{k m}^{i}\left({ }_{P}^{s} P^{\mathrm{T}(B)}\right)_{j l}^{m}-\left(\stackrel{s}{P}^{\mathrm{T}(B)}\right)_{l m}^{i}\left(P^{s} \mathrm{~T}(B)\right)_{j k}^{m} \\
= & B^{2}\left[\pi\left(r_{i k} r_{j l}-r_{i l} r_{j k}\right)+\left(r_{i k} B_{j} B_{l}-r_{i l} B_{j} B_{k}\right)+\pi^{2}\left(B_{i} B_{k} r_{j l}-B_{i} B_{l} r_{j k}\right)\right] \\
& M_{(R i)}^{\mathrm{T}(B)} B^{2}\left[\left(3 \pi+\pi^{2}\right) r_{j l}+\left(3-\pi^{2}\right) B_{j} B_{l}\right] \\
= & \operatorname{det}\left[M_{(R i) j l}^{\mathrm{T}}\right]=-3(B)^{8} \pi^{3}(3+\pi)^{3}(1+\pi)=0 \text { for } \pi=0
\end{aligned}
$$

Instead of $\pi=0$, we could take $\pi=-3$, which also makes the determinant zero. The $\pi=-1$ is not allowed, because with requirement $\pi_{2}=-3-3 \pi_{1}$, the presentation (26) is not possible.

On the other hand similar calculations for the vector $\mathbf{G}$ yield this result:

$$
\begin{aligned}
\stackrel{s}{P}_{i j k}^{\mathrm{T}(G)} \equiv & G_{j} r_{i k}+G_{k} r_{i j}+\pi_{1} G_{i} r_{j k}+\pi_{2} \frac{G_{i} G_{j} G_{k}}{(G)^{2}} ; M_{i j k l}^{\mathrm{T}(G)} \\
\approx & \left(\stackrel{s^{\mathrm{T}(G)}}{P}\right)_{k m}^{i}\left(\stackrel{s^{\mathrm{T}(G)}}{P}\right)_{j l}^{m}-\left(\stackrel{s^{\mathrm{T}(G)}}{P}\right)_{l m}^{i}\left(\stackrel{s}{P}{ }^{\mathrm{T}(G)}\right)_{j k}^{m} \\
= & G^{2}\left[\pi_{1}\left(r_{i k} r_{j l}-r_{i l} r_{j k}\right)+\left(1+\pi_{2}\right)\left(r_{i k} U_{j} U_{l}-r_{i l} U_{j} U_{k}\right)\right. \\
& \left.+\pi_{1}\left(\pi_{1}+\pi_{2}\right)\left(U_{i} U_{k} r_{j l}-U_{i} U_{l} r_{j k}\right)\right] \\
= & M_{(R i) j l}^{\mathrm{T}(G)} G^{2}\left[\left(3 \pi_{1}+\pi_{1}^{2}+\pi_{1} \pi_{2}\right) r_{j l}+\left(3+3 \pi_{2}-\pi_{1}^{2}-\pi_{1} \pi_{2}\right) U_{j} U_{l}\right]
\end{aligned}
$$

with $\pi_{2}=-3\left(\pi_{1}+1\right)$

$$
\text { and } \pi_{1}^{2}-3 \pi_{1}-3=0=>\left(M_{(R i)}^{\mathrm{T}(g)}\right)_{j l}=-2 \pi_{1}^{2} G^{2} r_{j l} \operatorname{det}\left[M_{(R i) j l}^{\mathrm{T}}\right] \neq 0
$$

And so we identify vector $\mathbf{G}$ with gravitational vector. It is associated with every mass-particle (electron, proton, etc.) and, as we postulated in the beginning of the paper, its value is accumulated to reach a very large number. So it must be a time vector. If it were a space-vector then for closed Universe (3D share with respect to spacial coordinates) it would cancel itself out and would have the value zero, which would be a contradiction of our postulate.

For any given "isolated" (non interacting with other particles) system of particles the value of the gravitational field depends on two things: a) its value at infinity-the back-ground value associated with all the particles outside the system and b) by the value of the mass-particle that are in consideration (such as Solar System). The square magnitude of this vector $G^{2}$ as we postulated before defines the value of Newton Gravitational Constant accordingly this expression: $K_{N}=\frac{c^{3}}{\hbar G^{2}}$.

As far as the Electromagnetic field, its identification is not as straight forward as one would hope and as we will discuss it in more details in Section 8-Electromagnetic and Other Vectors", but for now we choose the vector $\mathbf{E}$ to represent Electromagnetic field.

The 3-index tensors $\mathbf{N}$ and $\mathbf{C}$ should be associated with mass-matter such 
as electron, protons, etc. We must emphasize the fact that this is a field approach to the description of Matter, which requires that these functions were strongly localized.

Jumping ahead we can say that equations of motion for these functions ( $\mathbf{N}$ and $\mathbf{C}$ ) are strictly non linear due to the fact that tensor of Total-Matter is proportional to a square of Tensor-Potential $\mathbf{P}^{\mathrm{T}}$ and thus square of $\mathbf{N}$ and C . The Lagrangian of the mass-matter, as one would expect, will be proportional to the square Total-Matter $\mathbf{M}^{\mathrm{T}}$, will contains terms of fourth power with respect to 3-index tensors $\mathbf{N}$ and $\mathbf{C}$. Instead of using two 3-index tensors $\mathbf{N}$ and $\mathbf{C}$ we-whenever it makes sense and specially in symbolic writing-will refer to a generic term $\mathbf{H}$ defined as a "pair" of tensors $\mathbf{H} \equiv(\mathbf{N} ; \mathbf{C})$. Or equivalently we can introduce one complex 3-index tensor $\mathbf{H}$ (and $\mathbf{H}$ for complex conjugate) with its real part to be $\mathbf{N}$ tensor and its imaginary part the tensor $\mathbf{C}$.

$$
H_{i j k} \equiv N_{i j k}+i C_{i j k} ; \quad \stackrel{*}{H} \text { ijk } \equiv N_{i j k}-i C_{i j k}=H_{i k j} \quad i^{2}=-1
$$

which gives an alternative way to write the equations for the mass-matter-similar to Quantum Mechanics.

The equations of motions for each variable can be obtained by means of variation of the Lagrangian density $L^{\sqrt{ }}$ with respect to each particular variablemetric tensor $\mathbf{r}, 4$ vectors $\mathbf{G}, \mathbf{B}, \mathbf{D}, \mathbf{E}$ and two 3 -index tensors $\mathbf{N}, \mathbf{C}$.

In general this equations are linear second order partial derivatives with respect to each variable. But they are non-linear with respect to the first order derivatives since $\mathbf{M}^{\mathrm{T}}$ comes in the " $n$ " $(n>2)$ power in Lagrangian (30). If we are interested to write equations for the Universe, we would have to do an averaging procedure for the matter (all fields), similarly to the way statistical physics derives its equations from basic Newton's laws and equations.

There are couple issues in above described scheme. The above equations don't look like the "typical" field theory equations of physics today, which are linear with respect to both second and first derivatives on unknown variable. There is also an issue of unknown parameters " $n$ " (maximum " $n$ ") and $\lambda_{n s}$ of expr. (30) - or just parameters $\lambda_{s}$ if " $n$ " is fixed to one value (say $n=4$-which determine the exact expression for the Lagrangian.

Both of this problems could be rectified if we try to consider a "small" isolated system of particles and try to simplify the Lagrangian (30) using a "small" factor-small number of particle with respect to the number of particle in Galaxy or Universe $\left(N_{\text {sys }} \ll N_{\text {gal }}\right)$. The original Lagrangian presented as a series by parameter $N_{\text {sys }} / N_{\text {gal }}$. should lead to a standard Lagrangian of known in physic theories: Maxwell theory in case of Electromagnetism (vector field E) and Einstein type equations (may be with the dark matter) for the metric tensor $\mathbf{r}$.

It is natural to assume that in this procedure-transferring from general Lagrangian to a "small system" Lagrangian-there will be some "unwanted" and/or unphysical terms that should be vanishing. This will set some requirements on the parameters $\lambda_{n s}$, which should lead to a set of equations on these $\lambda$ 's that 
will determine their actual values.

What important here is that this procedure will also deliver Lagrangians (and thus equations) for "other" 4 fields-2 vectors B , D , and two 3 -index tensors $\mathbf{N}, \mathbf{C}$. And that is the benefit of the "Unification" procedure.

In this scheme (procedure) we are allowed to eliminate some terms (almost at will), but we are not allowed to add any terms that we would like to have. The Eddington Affine unification is the only basis for all the terms that could be available in the final field theory of the Matter. And as we will show it would be sufficient enough to generate both Gravitational and Electromagnetic theories. It also will generate the field theory (albeit non linear) for the mass-matter (proton, electron, etc.).

Per our main postulate that the Gravitational vector $\mathbf{G}$ has much greater value than any other field, we can separate the Total-Matter Ricci tensor$\mathbf{M}_{(R i)}^{\mathrm{T}} \equiv M_{(R i) j l}^{\mathrm{T}}$-on large Gravitational field $\mathbf{G}$ and the "conventional" Matter $\mathbf{M}_{(R i)}$ and write it as a series with respect to the parameter $\mathbf{G}\left(G=\sqrt{G_{i} G_{j} r^{i j}}\right)$ :

$$
\begin{aligned}
M_{(R i) j l}^{\mathrm{T}}= & G^{2} \pi_{1}\left(3+\pi_{1}+\pi_{2}\right) r_{j l} \\
& +\left(3+3 \pi_{2}-\pi_{1}^{2}-\pi_{1} \pi_{2}\right) U_{j} U_{l}+G Q_{(R i) j l}(\mathbf{P})+M_{(R i) j l}(\mathbf{P})
\end{aligned}
$$

where $Q_{j l}$ is a tensor proportional Tensor-Potential $\mathbf{P}$, which represent the Matter in a way we understand it-that is all the Matter $\mathbf{M}^{\mathrm{T}}$ without the Gravitational vector $\mathbf{G}$.

So for the "small system" of isolated Matter (or in vacuum, where $\mathbf{P}=0$ ) in the first order of approximation the Ricci contraction $\left(\mathbf{M}_{(R i)}^{\mathrm{T}}\right)$ has this expression:

$$
M_{(R i) j l}^{\mathrm{T}} \approx G^{2} \pi_{1}\left(3+\pi_{1}+\pi_{2}\right)\left[r_{j l}+\frac{\left(3+3 \pi_{2}-\pi_{1}^{2}-\pi_{1} \pi_{2}\right)}{\pi_{1}\left(3+\pi_{1}+\pi_{2}\right)} U_{j} U_{l}\right] .
$$

It would seem to be logical to choose parameters $\pi_{1}$ and $\pi_{2}$ to be such that the term proportional to $U_{j} U_{l}$ vanishes.

$$
\pi_{2}=-\frac{\pi_{1}^{2}-3}{\pi_{1}-3} \text { and } M_{(R i) j l}^{\mathrm{T}} \approx-G^{2} \frac{6 \pi_{1}}{\pi_{1}-3} r_{j l}
$$

In this case the Lagrangian and the equations of motion for the TensorPotential $\mathbf{P}$ in the first order of approximation are governed by metric tensor $\mathbf{r}$. If we add to this requirement an other one: $\pi_{2}=-3\left(\pi_{1}+1\right)-$ which as we will see in the section "Electromagnetic and Other Vector Fields" is dictated by the desire to obtain description for Electromagnetic field in Maxwell form (or almost Maxwell form)-we will have this equation for $\pi_{1}$ constant: $\pi_{1}^{2}-3 \pi_{1}-3=0$.

As we will see it later, the truly must requirement out above two is the last one: $\pi_{2}=-3\left(\pi_{1}+1\right)$. The first requirement $\pi_{1}^{2}-3 \pi-3=0$ it's not that important, but it makes the presentation a visibly "better looking".

In the end of this section we would like to note that if more rigorous analysis shows that 16 equations (29) is not enough for complete definition of the metric 
$\mathbf{r}$, we can always add 4 more linear equations corresponding to setting the vector $\mathbf{B}$ to zero.

\section{Lagrangian of a "Small System" of Matter}

In this section we will derive the Lagrangian of a "small system" of Matter and by doing so try to determine the requirements for determining the $\lambda$-parameters of the general Lagrangian (30).

We begin this procedure with setting a small parameter and writing the Lagrangian in the series with respect to it. In our case the small parameter is $N_{\text {sys }} / N$, where $N_{\text {sys }}$ is the number of particles in the "small system" and $N$ is the number of particles outside of our "small system" that create the background metric. The small parameter can also be expressed in terms of Gravvector $\mathbf{G}$. Our main postulate is that the gravitational field generated by all particles accumulates and reaches very high value. "Small" here means that the collective gravitational field of the "small" system is much smaller than the value of the gravitational field created by all the particle surrounding (outside) our "small" test system. In this case gravitational field of the outside (the Galaxy or the Universe) particles could be considered constant and equal its value $\mathbf{G}_{\infty}$ at infinity of "small system". The total then Gravitational field $\mathbf{G}$ of our "small system" can be written as $\mathbf{G}_{\infty}+\overline{\mathbf{G}}$ and $\overline{\mathbf{G}} \ll \mathbf{G}_{\infty}$.

As the first step of the procedure we should write the tensor of Total-Matter $\left(\mathbf{M}^{\mathrm{T}}\right)$ and Ricci Total-Matter tensor $\mathbf{M}_{(R i)}^{\mathrm{T}}$, defined by expr. (9) as a series by parameter $\mathbf{G}$ - the magnitude of vector $\mathbf{G}$ thus separating out large Gravitational field and the rest of the Matter. For tensor potential $\mathbf{P}^{\mathrm{T}}$ we have:

$$
P_{j k}^{\mathrm{T} i}=P_{j k}^{i}+P_{j k}^{(G) i} \text { where } P_{i j k}^{(G)} \equiv P_{j k}^{(G) m} r_{i m}=G_{j} r_{i k}+G_{k} r_{i j}+\pi_{1} G_{i} r_{j k}+\pi_{2} \frac{G_{i} G_{j} G_{k}}{G^{2}}
$$

where $\mathbf{P} \equiv P_{i j k}$ is a Tensor-Potential of the Matter defined as the TensorPotential of the Total-Matter without the gravitational field $\mathbf{G}$. Using the above and the definition (30) we can produce the expression for the Total-Matter $\mathbf{M}^{\mathrm{T}}$ presented by the components that depend on vector $\mathbf{G}$ and the rest of the matter presented by the tensor $\mathbf{M}$ :

$\mathbf{M}^{\mathrm{T}}=G^{2} \mathbf{I}+G \mathbf{Q}+M(\mathbf{P})+\mathbf{G}^{\prime}+\mathbf{R}$ or $M_{i j k l}^{\mathrm{T}}=D^{2} I_{i j k l}+D Q_{i j k l}+M_{i j k l}+\left(G^{\prime}\right)_{i j k l}+R_{i j k l}$ with

$$
\begin{aligned}
& \mathbf{I} \equiv I_{i j k l}=\pi_{1}\left(r_{i k} r_{j l}-r_{i l} r_{j k}\right)+\left(1+\pi_{2}\right)\left(r_{i k} U_{j} U_{l}-r_{i l} U_{j} U_{k}\right) \\
& +\pi_{1}\left(\pi_{1}+\pi_{2}\right)\left(r_{j l} U_{i} U_{k}-r_{j k} U_{i} U_{l}\right) \\
& \begin{aligned}
\mathbf{G}^{\prime} \equiv G_{i j k l}^{\prime}= & \left(G_{j ; k} r_{j l}-G_{j ; l} r_{i k}\right)+\left(G_{i ; k} r_{j l}-G_{i ; l} r_{i k}\right)+\pi_{1}\left(G_{l ; k}-G_{k ; l}\right) r_{i j} \\
& +\pi_{2}\left[\left(\frac{G_{i} G_{j} G_{l}}{G^{2}}\right)_{; k}-\left(\frac{G_{i} G_{j} G_{k}}{G^{2}}\right)_{; l}\right]
\end{aligned} \\
& \mathbf{R} \equiv R_{i j k l}=r_{m i} R_{j k l}^{m} \\
& \text { and } R_{j k l}^{i}=\gamma_{j l, k}^{i}-\gamma_{j k, l}^{i}+\gamma_{m k}^{i} \gamma_{j l}^{m}-\gamma_{m l}^{i} \gamma_{j k}^{m} \gamma_{j k}^{i}=\frac{1}{2} r^{i m}\left(r_{m j, k}+r_{m k, j}-r_{j k, m}\right)
\end{aligned}
$$


where $\mathbf{U}$ is a unit vector of $\mathbf{G}, \mathbf{R} \equiv R_{j k l}^{i}$ is a standard Riemann geometric curvature tensor defined by metric of the space $\mathbf{r} \equiv r_{i j}$ and the tensor of Matter $\mathbf{M}$ is defined the same way as the tensor $\mathbf{M}^{\mathrm{T}}$ except to the presence of the Grav-vector $\mathbf{G}-\left.\mathbf{M}(\mathbf{P}) \equiv \mathbf{M}^{\mathrm{T}}\left(\mathbf{P}^{\mathrm{T}}\right)\right|_{\mathbf{G}=0}$.

Tensor $\mathbf{Q}$ is a linear function of the tensors $\mathbf{P}$ (or its patrs $\stackrel{s}{\mathbf{P}}$ and $\stackrel{a}{\mathbf{P}}$ ) and it is formed when we calculate the quadratic terms of expr. (9) $\left(\begin{array}{cc}s^{\mathrm{T} i} & s \mathrm{~T} m \\ P_{m k} & \stackrel{s_{j l}^{\mathrm{T}} i}{P_{j l}}-\stackrel{s}{P}_{m l} P_{j k}\end{array}\right)$ as well as covariant derivatives for $\stackrel{a}{\mathbf{P}}^{\mathrm{T}}$ associated with parameters $\mu_{1}$ thru $\mu_{7}$ (such as $\stackrel{s^{\mathrm{T}} i}{P}{ }_{j \mid k}-\stackrel{s}{P}_{j k \mid l}^{\mathrm{T} i}$ ). The exact expression for $\mathbf{Q}$ is rather lengthy and will be given later. The important part is that it linearly depends on the fully symmetric tensor $\mathbf{N}$, torsion type tensors $\mathbf{C}$ and depending on the choice of parameters $\mu$-s (as well as $\pi_{1}$ and $\pi_{2}$ ) it might also include the vectors $\mathbf{B}, \mathbf{D}, \mathbf{E}$.

The tensor of Matter is defined the same way as the tensor $M^{\mathrm{T}}$ except without the vector $\mathbf{G}$ and depends only on two traceless 3-index tensors $\mathbf{N}$, $\mathbf{C}$ and 3 vector field $\mathbf{B}, \mathbf{D}, \mathbf{E}$. At this point the exact expression for the tensor of Matter $\mathbf{M}$ as a function of the 3-index tensors $\mathbf{N}, \mathbf{C}$ and the vector field $\mathbf{B}, \mathbf{D}, \mathbf{E}$ is not important and and will be considered in more details in the following sections. However it needs to be mentioned that they are determined by (describe by) the "small system".

The expression for $\mathbf{M}^{\mathrm{T}}$ above seems to imply that values the terms $\mathbf{G}^{\prime}$ and $\mathbf{R}$ are of the same order of magnitude as the terms of the "small system" Matter M . This is actually not the case. According to Einstein's General Relativity (and there is no reason to doubt it as a first order approximation) the metric tensor $\mathbf{r}$ is a small deviation from the Minkowski space: $r_{00}=1-\frac{K_{N} N_{p} m_{p}}{c^{2} \rho}$, where $\rho$ is the distance from the Mass, $m_{p}$ is a proton's mass and $N_{p}$ is the number of particles. Metric is defined up to the constant and if we consider $r_{i j} / L_{p}^{2}$ instead of $r_{i j}$ (where $L_{p}$ is a Plank length), we will get this expression for the metric as a function of the distance $\rho$ [36]:

$$
r_{00}=\frac{c^{3}}{K_{N} \hbar}-\frac{N_{\text {sys }}}{\rho L_{a}}=\frac{1}{L_{p}^{2}}-\frac{N_{\text {sys }}}{\rho L_{a}} \quad \text { where } \quad L_{a}=\frac{\hbar}{c m_{p}} \text { is atomic length }
$$

This expression gives a good (except for the sign) physical description of the metric. It splits it on two part: metric at an infinity and the metric due to the presence of the matter only-the form one would expect to see in any linear approximation. From the expr. (41) follows that written in this form the metric tensor $\bar{r}$ has units $1 / \mathrm{cm}^{2}$, which well with an agreement with the Einstein equation for the $\bar{r}_{i j}:\left(\partial \partial \bar{r}_{i j}=T_{i j}\right)$, where Energy-Momentum tensor is expressed in the units $\hbar c$ and thus has units $\left.1 / \mathrm{cm}^{4}\right)$. Another words the correct way to write approximate linearizion (with respect to the large parameter $G^{2}$ ) of metric is $r_{i j}=\delta_{i j}+\frac{\bar{r}_{i j}}{G^{2}}$, where $\bar{r}_{i j}$ is the metric defined by the "small system". From this follows that the Riemann tensor $\mathbf{R} \equiv R_{i j k l} \approx \bar{R}_{i j k l} / G^{2}$. So if we intro- 
duce a new tensor $\mathbf{R}^{G}=G^{2} \mathbf{R}$ it will be of the order of magnitude of the Matter that created it. So we can write the last term of (40) as:

$$
R_{i j k l} \approx \frac{R_{i j k l}^{G}}{G^{2}} \text { where } R_{i j k l}^{G}=G^{2} R_{i j k l} \text { or symbolically } \mathbf{R}=\frac{\mathbf{R}^{G}}{G^{2}} .
$$

We should expect similar situation, if consider a gravitational field $\mathbf{G}$, that is extremely large at infinity (created by outside mass-Galaxy or Universe). But if we write a similar expression for vector $\mathbf{G}$ (for its time component $G_{0}$ ), which has units of $\frac{1}{\text { length }}$, we run into a bit of trouble:

$$
G_{0}=\frac{1}{L_{p}}+\frac{N_{\mathrm{sys}}}{\rho} . L_{p}=\sqrt{\frac{c^{3}}{K_{N} \hbar}} \text { is Plank length. }
$$

Substituting in this formula values for our Solar system $-10^{57}$ number of particles of Sun, $10^{8} \mathrm{~km}$ for the distance to the Sun-we will get that "an addition" due to Solar system is several orders of magnitude higher that the value at infinity: $1 / L_{P}=10^{33} 1 / \mathrm{cm}$ and $N / \rho=10^{57} / 10^{13}(1 / \mathrm{cm})=10^{44}$.

This problem can be removed is we assume that the vector that defines the "addition" of Grav-field is not the vector $\mathbf{G}$, but its "square" $S_{i}=G G_{i}$. In this case for the total value of the vector $\mathbf{S}$ we have the expression similar to the metric tensor component $r_{00}$ :

$$
S_{0}=S_{\infty}+\frac{N_{\text {sys }}}{\rho L_{a}}, \quad \text { where } S_{\infty}=\frac{1}{L_{p}^{2}} ; \quad L_{a}=\frac{\hbar}{c m_{p}} .
$$

Taking this into a count we can rewrite $\mathbf{G}^{\prime}$ in the form $\mathbf{S}^{\prime}$ that reflects its proper value with respect to the magnitude of the vector $\mathbf{G}$.

$$
\begin{gathered}
P_{i j k}^{(G)} \equiv G_{j} r_{i k}+G_{k} r_{i j}+\pi_{1} G_{i} r_{j k}+\pi_{2} G U_{i} U_{j} U_{k} \\
=\frac{S_{j} r_{i k}+S_{j} r_{i k}+\pi_{1} S_{j} r_{i k}+\pi_{2} S U_{i} U_{j} U_{k}}{G} \equiv \frac{P_{i j k}^{(S)}}{G} \\
\begin{aligned}
& M_{i j k l}^{\left(G^{\prime}\right)}=P_{i j l ; k}^{(G)}-P_{i j k ; l}^{(G)}=\left[\frac{P_{i j l}^{(S)}}{G}\right]_{; k}-\left[\frac{P_{i j k}^{(S)}}{G}\right]_{; l}=\frac{M_{i j k l}^{\left(S^{\prime}\right)}}{G} \\
& \text { where } M_{i j k l}^{\left(S^{\prime}\right)}=\left(S_{i ; k} r_{j l}-S_{i ; l} r_{i ; j k}\right)+\left(S_{j ; k} r_{i l}-S_{j ; l} r_{i k}\right)+\pi_{1}\left(S_{l ; k}-S_{k ; l}\right) r_{i j} \\
&+\pi_{2}\left[\left(S_{i ; k} U_{j}-S_{i ; l} U_{k}\right) U_{l}+\left(S_{j ; k} U_{l}-S_{j ; l} U_{k}\right) U_{i}+\left(S_{l ; k}-S_{k ; l}\right) U_{j} U_{j}\right. \\
&-\frac{1}{4}\left(U_{i} r_{j l}+U_{j} r_{i l}+\pi_{1} U_{l} r_{i j}+\pi_{2} U_{i} U_{j} U_{l}\right) S_{m ; k} U^{m} \\
&+\frac{1}{4}\left(U_{i} r_{j k}+U_{j} r_{i k}+\pi_{1} U_{k} r_{i j}+\pi_{2} U_{i} U_{j} U_{k}\right) S_{m ; l} U^{m}
\end{aligned}
\end{gathered}
$$

In this form the term $\mathbf{S}^{\prime} \equiv M_{i j k l}^{\left(S^{\prime}\right)}$ is defined by the "small system" in consideration and the factor $\frac{1}{G} \approx \frac{1}{G_{\infty}}$ defines the "scale" as compared to the "small system" terms. Taking into considerations expr. (42) and (45), we can write the tensor of Total-Matter $\mathbf{M}^{\mathrm{T}}$ (expr. (40 line 1) in this symbolic form: 


$$
\mathbf{M}^{\mathrm{T}}=G^{2} \mathbf{I}+G \mathbf{Q}+\mathbf{M}+\frac{1}{G} \mathbf{S}^{\prime}+\frac{1}{G^{2}} \mathbf{R}^{G} .
$$

Thoughout this paper we will use square-vector $\mathbf{S}$ when we are dealing with $\mathbf{S}^{\prime}$ variable and we will continue to use scalar $\mathbf{G}\left(G^{2}=G_{i} G^{i}\right)$ to indicate the scale in the series decomposition.

The next step in writing the Lagrangian (30) is to calculate the Ricci $\mathbf{M}_{(R i)}^{\mathrm{T}}$ for $\tilde{\mathbf{M}}^{\mathrm{T}}$

$$
\mathbf{M}_{(R i) j l}^{\mathrm{T}}=\frac{1}{2}\left(M_{j m l}^{\mathrm{T} m}+M_{l m j}^{\mathrm{T} m}\right) .
$$

The tensor $\mathbf{M}_{(R i)}^{\mathrm{T}}$ can be calculated using expr. (46) and symbolically written as:

$$
\mathbf{M}_{(R i)}^{\mathrm{T}}=G^{2}\left[\mathbf{I}_{(R i)}+\frac{\mathbf{Q}_{(R i)}}{G}+\frac{\mathbf{M}_{(R i)}}{G^{2}}+\frac{\mathbf{S}_{(R i)}^{\prime}}{G^{3}}+\frac{\mathbf{R}_{(R i)}^{G}}{G^{4}}\right]
$$

where all " $(R i)$ " tensors are 2-index symmetrical tensors. For $\mathbf{I}$ and $\mathbf{I}_{(R i)}$ tensors we have:

$$
\begin{gathered}
\mathbf{I} \equiv I_{i j k l}=G^{2}\left[\pi_{1}\left(r_{i k} r_{j l}-r_{i l} r_{j k}\right)+\left(1+\pi_{2}\right)\left(r_{i k} U_{j} U_{l}-r_{i l} U_{j} U_{k}\right)\right. \\
+\pi_{1}\left(\pi_{1}+\pi_{2}\right)\left\{\left(U_{i} U_{k} r_{j l}-U_{i} U_{l} r_{j k}\right)\right] \\
\mathbf{I}_{(R i)}=\left[3 \pi_{1}+\pi_{1}\left(\pi_{1}+\pi_{2}\right)\right]\left[r_{j l}+\frac{3+3 \pi_{2}-\pi_{1}\left(\pi_{1}+\pi_{2}\right)}{3 \pi_{1}+\pi_{1}\left(\pi_{1}+\pi_{2}\right)} U_{j} U_{l}\right] .
\end{gathered}
$$

And using $\pi_{2}=-\frac{\pi_{1}^{2}-3}{\pi_{1}-3}$ we get these expressions for $\mathbf{I}$ and $\mathbf{I}_{(R i)}$ :

$$
\begin{gathered}
\mathbf{I} \equiv I_{i j k l}=G^{2} \pi_{1}\left[\left(r_{i k} r_{j l}-r_{i l} r_{j k}\right)+\left(1-\pi_{1}\right)\left(r_{i k} U_{j} U_{l}-r_{i l} U_{j} U_{k}\right)\right. \\
+\frac{3\left(1-\pi_{1}\right)}{\pi_{1}-3}\left\{\left(U_{i} U_{k} r_{j l}-U_{i} U_{l} r_{j k}\right)\right] \\
\mathbf{I}_{(R i)}=-8 \pi_{1} r_{j l} .
\end{gathered}
$$

And finally in order to write the Lagrangian (30) we need to calculate the $\operatorname{det}\left(\mathbf{M}_{(R i)}^{\mathrm{T}}\right)$. For our purposes it would be enough to have its first two orders of approximation: The expression for $\operatorname{det}\left(\mathbf{M}_{(R i)}^{\mathrm{T}}\right)$ can be explicitly calculated using the above expressions:

$$
\operatorname{det}\left(\mathbf{M}_{(R i)}^{\mathrm{T}}\right)=G^{2}\left[K_{\mathbf{I}}+\frac{K_{\mathbf{Q}}}{G}+\frac{K_{\mathbf{Q}^{2}}+K_{\mathbf{M}}}{G^{2}}+\frac{1}{G^{3}}(\cdots)\right]
$$

where $\mathbf{K}_{\mathbf{I}}, K_{\mathbf{Q}}, K_{\mathbf{Q}^{2}}$ and $K_{\mathbf{M}}$ are all invariants of appropriate terms of the $\mathbf{M}_{(R i)}^{\mathrm{T}}$ tensor. We note that in the expression above the invariant $K_{\mathbf{I}}$ after of being pulled out square root could be simply omitted. This effectively the same as setting $\mathbf{K}_{\mathbf{I}}$ to 1 . Also we need to point here that because in the expr. (48) terms containing $\mathbf{S}^{\prime}$ come only with a factor $1 / \sqrt{G^{3}}$, as it will be shown later, could be ignored.

Using all that we indicated above we can now calculate the Lagrangian density for the "small system" as a series by parameter $\mathbf{G}$ : 


$$
\begin{aligned}
L^{\sqrt{ }} & =\sum_{n=1,2 \cdots s=\text { allinv }} \lambda_{n s}\left(\mathbf{M}^{\mathrm{T}}\right)^{n}\left(\mathbf{M}_{(R i)}^{\mathrm{T}}\right)^{-n} \sqrt{\operatorname{det}\left(\mathbf{M}_{(R i)}^{\mathrm{T}}\right) \mid} \\
& =\sum_{n, s=\text { inv }} \lambda_{n s} G^{4}\left[1+\frac{K_{\mathbf{Q}}}{G}+\frac{K_{\mathbf{M}}}{G^{2}}\right]_{x}\left[\mathbf{I}+\frac{\mathbf{Q}}{G}+\frac{\mathbf{M}}{G^{2}}+\frac{\mathbf{S}^{\prime}}{G^{3}}+\frac{\mathbf{R}^{\mathbf{G}}}{G^{4}}\right]^{n}\left[r+\frac{\mathbf{Q}_{(R i)}}{G}+\frac{\mathbf{M}_{(R i)}}{G^{2}}+\frac{\mathbf{S}_{(R i)}^{\prime}}{G^{3}}+\frac{\mathbf{R}_{(R i)}^{\mathbf{G}}}{G^{4}}\right]^{-n}
\end{aligned}
$$

Opening all the square parentheses we can present the Lagrangian density in explicit form of series with respect to parameter $\mathrm{G}$.

First we need to point out that since $\mathbf{I}$ is a tensor compose of the metric tensor $r_{i j}$ and a pair of unit vectors $U_{i} U_{j}$, its any power ( $\mathbf{I}^{n}-$ any $n$ ) also will be compose of of the same two tensors $\mathbf{r}$ and $\mathbf{U U}$. Another words, in symbolic writing $\mathbf{I}^{n}=\mathbf{I}$. In second, since " $(\mathrm{Ri})$ ” is a form contraction, then writing in symbolic form we can drop the "(Ri)" identification. It is not difficult to see that the final expression (in symbolic writing) has the same form for any " $n$ ". As we will see in the next paragraphs there will be some unwanted terms that we would require to vanish. By increasing the number " $n$ " we increase the number $\lambda_{n s}$ of coefficients and thus make it possible to vanish those unwanted terms:

$$
\begin{aligned}
L^{\sqrt{ } \approx} & \sum_{n} \sum_{s=\text { inv }} \lambda_{n s}\left[G^{4}+G^{3} \mathbf{Q}+G^{2}\left(\mathbf{M}+\mathbf{Q}^{2}\right)+G\left(\mathbf{S}^{\prime}+\mathbf{M} \mathbf{Q}+\mathbf{Q}^{3}\right)\right]_{\mathbf{A}} \\
& +\left\{\mathbf{M}^{2}+\mathbf{M} \mathbf{Q}^{2}+\mathbf{Q}^{4}+\mathbf{R}^{\mathbf{G}}+\left[\mathbf{S}^{\prime} \mathbf{Q}\right]_{\mathbf{B}}\right\}+\frac{1}{G}\left\{\left[\mathbf{Q}^{3} \mathbf{M}+\mathbf{Q} \mathbf{M}^{2}\right]_{C}+\left[\mathbf{S}^{\prime}\left(\mathbf{Q}^{2}+\mathbf{M}\right)+\mathbf{Q R}^{\mathbf{G}}\right]_{\mathbf{D}}\right\} \\
& +\left\{\frac{\left.\left[\mathbf{M}^{3}+\mathbf{Q}^{2} \mathbf{M}^{2}\right]_{F}+\left(\mathbf{S}^{\prime}\right)^{2}+\mathbf{S}^{\prime}\left(\mathbf{Q} \mathbf{M}+\mathbf{Q}^{3}\right)+\mathbf{R}^{\mathbf{G}}\left(\mathbf{M}+\mathbf{Q}^{2}\right)\right]}{G^{2}}\right\}+\frac{[\cdots]}{G^{3}}
\end{aligned}
$$

where we labeled some bracket with a sub-script $[\cdots]_{\mathrm{A}},[\cdots]_{\mathrm{B}}$, etc. for easier identification.

The main experimental fact of the physics today is that the law of nuclear physics do not depend on the gravitational field. Meaning that if we set a (non moving) lab near our Sun or on a satellite at the edge of Solar system we will observe the same (or almost the same) physics law and measure the same proton mass and charge and the same quantum levels for hydrogen as we see them on Earth.

That means that the most significant term in the Lagrangian above should be the terms with the square of first derivatives of Tensor-Potential $\mathbf{P}\left(\mathbf{P}^{\prime}\right)$ or the terms $\mathbf{M}^{2}$. That also means that all the terms in the square bracket $[\cdots]_{A}-$ which much higher order of magnitude-must vanish. This can be achieved by choosing appropriate constants $\lambda_{n s}$ and $\mu$-s of expr. (53). After dropping $[\cdots]_{A}$ terms, we get this expression for the Lagrangian density:

$$
\begin{aligned}
L^{\sqrt{ }} \approx & \left\{\sum_{n, s=i n v} \lambda_{n s}\right\}\left[\mathbf{M}^{2}+\mathbf{M} \mathbf{Q}^{2}+\mathbf{Q}^{4}+\mathbf{R}^{\mathbf{G}}\right]+\left[\mathbf{S}^{\prime} \mathbf{Q}\right]_{\mathbf{B}} \\
& +\frac{\left[\mathbf{Q}^{3} \mathbf{M}+\mathbf{Q M}^{2}\right]_{\mathbf{C}}+\left[\mathbf{S}^{\prime}\left(\mathbf{Q}^{2}+\mathbf{M}\right)\right]_{\mathbf{D}}}{G}+\left[\mathbf{Q} \mathbf{R}^{\mathbf{G}}\right]_{\mathbf{E}} \\
& +\left\{\frac{\left.\left[\mathbf{M}^{3}+\mathbf{Q}^{2} \mathbf{M}^{2}\right]_{\mathbf{F}}+\left(\mathbf{S}^{\prime}\right)^{2}+\mathbf{S}^{\prime}\left(\mathbf{Q} \mathbf{M}+\mathbf{Q}^{3}\right)+\mathbf{R}^{\mathbf{G}}\left(\mathbf{M}+\mathbf{Q}^{2}\right)\right]}{\left.\mathbf{G}^{\mathbf{2}}\right\}+\frac{[\cdots]}{G^{3}}}\right.
\end{aligned}
$$

Similarly, the most important terms that contain square $\mathbf{S}^{\prime}$ (the quadratic 
terms) are proportional to the factor $1 / G^{2}$. However, there are $\mathbf{S}^{\prime}$ terms that exist in the brackets $[\cdots]_{\mathrm{B}}$ and $[\cdots]_{\mathrm{D}}$, which come into the Lagrangian with much higher factor of the parameter $\frac{1}{G^{2}}$. For that reason all the terms in the brackets $[\cdots]_{\mathbf{B}}$ and $[\cdots]_{\mathbf{D}}$ must vanish. In addition, because the equations of motion for the Tensor-Potential $\mathbf{P}$ is defined by the terms $\mathbf{M}^{2}$, the terms in the square bracket $[\cdots]_{\mathrm{C}}$ and $[\cdots]_{\mathrm{F}}$ which come with much smaller factor $1 / G$ and $1 / G^{2}$ respectively could be dropped as well as the all terms with the factor $1 / G^{3}$ and smaller. And finally, as we will show few paragraphs below, the Lagrangian $\mathbf{R}^{\mathbf{G}}$ is of order $1 / G^{2}$. However the terms $\left[\mathbf{Q R}^{\mathbf{G}}\right]$ in the bracket $[\cdots]_{\mathrm{E}}$ come with a factor $1 / G$ and thus much higher order. This means that the terms in the bracket $[\cdots]_{E}$ must vanish. Not all these additional requirements are new. For example, it can be shown (as we will see in few paragraphs later) that using partial integration the the terms $\mathbf{S}^{\prime} \mathbf{Q}$ could be written as a sum of the terms in the brackets $[\cdots]_{\mathrm{A}}$ and the terms proportional to $1 / G^{2}$.

After dropping the "unwanted" terms and restoring the summations by "power $n$ " and by all invariants, we will have this expression for the Lagrangian of the Total-Matter of the "small system":

$$
\begin{aligned}
L \approx & \sum_{n=1,2 \ldots s=\text { inv }} \lambda_{n s} \lambda^{(M)}\left(\mathbf{M}^{2}+\mathbf{M} \mathbf{Q}^{2}+\mathbf{Q}^{4}\right)+\sum_{n=1,2 \ldots s=\mathrm{inv}} \sum_{n s} \lambda_{n}^{(R)} \mathbf{R}^{\mathbf{G}} \\
& +\sum_{n=1,2 \ldots s=i n v} \sum_{n s} \lambda_{n}^{\left(S^{\prime} R\right)} \frac{\left(\mathbf{S}^{\prime}\right)^{2}+\mathbf{S}^{\prime}\left(\mathbf{M Q}+\mathbf{Q}^{3}\right)+\mathbf{R}^{\mathbf{G}}\left(\mathbf{Q}^{2}+\mathbf{M}\right)}{G^{2}}
\end{aligned}
$$

In the expression above we sub-divided the Lagrangian on three groups of terms separated by $\Sigma$ signs. The first group represents the Lagrangian of the Matter, the second one is similar to Einstein Lagrangian and includes the curvature tensor $\mathbf{R}$ (actually $\mathbf{R}^{\mathbf{G}}=\mathbf{G}^{2} \mathbf{R}$ ) by itself and the third group has the terms $\mathbf{S}^{\prime 2}$ as well as the curvature terms in a product with "mass-matter" terms. The latter comes with a very small factor $1 / G^{2}$. We must remember that all invariants in expr (55) are formed by either metric tensor $\mathbf{r} \equiv r^{i j}$ or by a pair of $\mathrm{u}$

vectors $U^{i} U^{j}$ or by both simultaneously (ex. $R_{i j k l} r^{j k} U^{i} U^{l}$ ). The second group of terms consists of only two terms: $\lambda_{1}^{(R)} \mathbf{R}^{\mathbf{G}} \equiv \lambda_{1}^{(R)} G^{2} R_{j l} r^{j l}$ and $\lambda_{2}^{(R)} \mathbf{R}^{\mathbf{G}} \equiv \lambda_{2}^{(R)} G^{2} R_{i j} U^{i} U^{j}$. It is not difficult to show that the term $\mathbf{R}_{j k}^{\mathbf{G}} U^{i} U^{k}$ belongs to the terms $\left(\mathbf{S}^{\prime}\right)^{2}$ and thus could be combined with them, or effectively dropped. The term, $\lambda_{1}^{(R)} R$, is in fact the Einstein scalar curvature invariant. It is important to point out that existence of this term is absolutely essential. With out it, as can shown (see section "Gravitation"), the equations of motions for the metric $r$ are incomplete. So before we continue, we need to demonstrate that there is at least one invariant in the expr. (30) that yields the Einstein invariant $G^{2} R$. And because we are interested only in terms $\mathbf{R}^{\mathbf{G}}$ we can consider it in vacuum or effectively drop all other variables (set $\mathbf{P}$ as well $\mathbf{S}^{\prime}$ to zero). 
Let us consider the following second order $(n=2)$ invariant with

$$
\begin{aligned}
& \pi_{2}=-3-3 \pi_{1} \pi_{1}^{2}-3 \pi_{1}-3=0: \\
&\left.\left.L^{\sqrt{ }}=\mathbf{M}_{j k l}^{\mathrm{T} i} \mathbf{M}_{m n i}^{\mathrm{T} l}(\mathbf{M})_{(R i)}^{-1}\right)^{j k}\left(\mathbf{M}^{\mathrm{T}}\right)_{(R i)}^{-1}\right)^{m n} \sqrt{\operatorname{det}\left|\left(\mathbf{M}_{(R i)}\right)\right|} \text { where } \\
& \mathbf{M}_{i j k l}^{\mathrm{T}}=G^{2}\left\{I_{i j k l}+\frac{R_{i j k l}}{G^{2}}\right\} ; \mathbf{M}_{(R i) j l}^{\mathrm{T}} \equiv M_{i j k l} r^{i k}=G^{2}\left\{-2 \pi_{1} r_{j l}+\frac{R_{j l}}{G^{2}}\right\} \text { and } \\
& I_{i j k l}=G^{2}\left\{\begin{array}{r}
\pi_{1}\left(r_{i k} r_{j l}-r_{i l} r_{j k}\right)+\left(1+\pi_{2}\right)\left(r_{i k} U_{j} U_{l}-r_{i l} U_{j} U_{k}\right) \\
\left.\quad+\pi_{1}\left(\pi_{1}+\pi_{2}\right)\left(U_{i} U_{k} r_{j l}-U_{i} U_{l} r_{j k}\right)\right\}
\end{array}\right.
\end{aligned}
$$

Our goal here is to calculate the linear with respect to Riemann curvature $\mathbf{R}$ terms. Strait forward calculations give this result:

$$
\begin{aligned}
L & \approx\left[2 I_{j k l}^{i} R_{m n i}^{l} r^{j k} r^{m n}-\frac{2}{\hat{\pi}} I_{j k l}^{i} I_{m n i}^{l} r^{j k} R_{p q} r^{m p} r^{n q}\right] \\
& =-2\left[\hat{I}_{l}^{i} R_{i}^{l}+\frac{2}{\hat{\pi}} \hat{I}_{l}^{i} I_{m n i}^{l} r^{j k} R_{p q} r^{m p} r^{n q}\right] \text { where } \\
\hat{\pi} & =-\frac{6 \pi_{1}}{\pi_{1}-3} \quad \hat{I}_{l}^{i} \equiv I_{j k l}^{i} l^{j k}=\pi_{\delta} \delta_{l}^{i}+\pi_{U} U^{i} U_{l} ; \quad \pi_{\delta}=\pi_{1}\left(3+\pi_{1}+\pi_{2}\right) ; \\
\pi_{U} & =3\left(1+\pi_{1}\right)+\pi_{1}\left(\pi_{1}+\pi_{2}\right)
\end{aligned}
$$

Continue with calculation of $L$ we get:

$$
\begin{aligned}
L & \approx-2\left[\pi_{\delta} R+\pi_{U} R_{i j} U^{i} U^{j}+\frac{1}{\hat{\pi}}\left(-\pi_{\delta} \hat{\pi} R+\pi_{U} U^{l} U_{i} I_{j k l}^{i} R^{j k}\right)\right] \\
& =-2\left[\pi_{U} R_{i j} U^{i} U^{j}+\frac{\pi_{U}}{\hat{\pi}} U^{l} U_{i} I_{j k l}^{i} R^{j k}\right] \\
& =-2\left[\pi_{U} R_{i j} U^{i} U^{j}+\frac{\pi_{U}}{\hat{\pi}} \pi_{1}\left(1+\pi_{1}+\pi_{2}\right)\left(R-U^{l} U_{i} R^{j k}\right)\right] \\
& =-2 \pi_{U}\left[\pi_{1} \frac{1+\pi_{1}+\pi_{2}}{\hat{\pi}} R+\left(1-\pi_{1} \frac{1+\pi_{1}+\pi_{2}}{\hat{\pi}}\right) R_{j k} U^{i} U^{k}\right]
\end{aligned}
$$

Thus we showed that the terms $G^{2} R$ is possible.

In addition this term has one more "interesting" property. Even though the tensor $G^{2} \mathbf{R}$ is about the same order of magnitude as the Matter of "small system" that defines it $-G^{2} \mathbf{R} \approx 1$ - the Lagrangian of scalar curvature- $G^{2} R$-is of much small order of magnitude $G^{2} R \approx 1 / G^{2}$ (as it is in General Relativity). The reason for that is that the highest values of of $G^{2} R$ are in second derivatives of metric, which can be converted (by way of partial integration) to a surface integral, or in a effect dropped. That leave the the Lagrangian $G^{2} R$ to be proportional to the square of first derivatives of metric and that means to be proportional to the $\left(1 / G^{2}\right)^{2}$, which makes that Lagrangian $G^{2} R$ to be of the same order of magnitude as the rems $\left(\mathbf{S}^{\prime}\right)^{2}$ Taking this into a account we can write the final expression for the Lagrangian of the Total-Matter as composition of the Lagrangian of the Matter and of the Gravitational Lagrangian:

$$
\begin{aligned}
L \approx & \left\{\sum_{s=\text { inv }} \lambda_{s}^{(M)}\left(\mathbf{M}^{2}+\mathbf{M} \mathbf{Q}^{2}+\mathbf{Q}^{4}\right)\right\}_{\text {Matter }} \\
+ & \left\{\lambda^{(R)} G^{2} R+\frac{1}{G^{2}} \sum_{s=\text { inv }} \lambda_{s}^{(S)}\left(\mathbf{S}^{\prime}\right)^{2}+\sum_{s=\text { inv }} \lambda_{s}^{(S M)} \mathbf{S}^{\prime}\left(\mathbf{M Q}+\mathbf{Q}^{3}\right)\right. \\
& \left.+\sum_{s=\text { inv }} \lambda_{s}^{(M r)} \mathbf{R}^{\mathbf{G}}\left(\mathbf{Q}^{2}+\mathbf{M}\right)\right\}_{\text {Grav }}
\end{aligned}
$$


where $\lambda_{s}^{(M)}, \lambda_{s}^{(R)}, \lambda_{s}^{(S)}, \lambda_{s}^{(S M)}$ and $\lambda_{s}^{(M r)}$ are constants, which are linear combinations of $\lambda_{n, s}$ of Lagrangian (30). The above Lagrangian could be written in a generic (phenomenological) form subdividing it on two sub-Lagrangians $L_{M}$-and $L_{\text {Grav }}$, each associated with the figure bracket of expr. (59) above with corresponding sub-indeces.

$$
S=\int \sqrt{-r} \mathrm{~d}^{4} x L ; L=L^{(M)}+\frac{L^{(\text {Grav })}}{G^{2}} ; \text { where } L^{(\text {Grav })}=L^{\left(S^{\prime}\right)}+L^{(M S)}+L^{(M r)}+L^{(r)}
$$

where $L^{(M)}$ includes the terms associated with all forms of Matter (except the Gravitational field $\mathbf{S}$ and the metric curvature $\mathbf{r}$ ) and the Lagrangian $L^{\text {(Grav) }}$ which includes gravitational square-vector $\mathbf{S}^{\prime}$ and the curvature tensor $\mathbf{R}$ built on the metric tensor $\mathbf{r}$. Both sub-Lagrangians are defined by "small system" (Matter M ) only. However the sub-Lagrangian $L^{\text {(Grav) }}$ comes with a very small factor $\frac{1}{G^{2}} \approx 10^{-66} \frac{1}{\mathrm{~cm}^{2}}$. This small factor for a "small system" serves as an universal Gravitational constant.

Such presentation of the Total-Matter Lagrangian $L$ on $L^{(M)}$ and $L^{\text {(Grav) }}$ parts is not exactly perfect in a sense that there are some interaction terms that sort of hidden from obvious view. For example the Lagrangian $L^{(M)}$ depends on metric $\mathbf{r}$ (albeit with a small factor $1 / G_{\infty}^{2}$ ). This dependence is of the same order of magnitude as the $L^{(\text {Grav })} / G^{2}$. Or dependence of $L(M)$ on the unity vector $\mathbf{U}$, which produces also terms similar to $L^{\text {(Grav) }} / G^{2}$.

The equation of motion are obtained by variation of the Lagrangian density $L \sqrt{-\operatorname{det}\left(r_{i j}\right)}$ with respect to the Tensor-Potential $\mathbf{P}$, Gravitational squarevector $\mathbf{S}$ and the space metric $\mathbf{r}$. One can use this Lagrangian written in covariant form to get the system of equations for each variable $\mathbf{N}, \mathbf{C}, \mathbf{B}, \mathbf{D}$ and $\mathbf{E}^{*}$. Such equations-that include metric $\mathbf{r}$ and covariant derivatives-might be particular beneficial when the "small system" become very large such as our Solar system or maybe a Galaxy where number of particles (and thus its Gravitational field) become comparable to the Gravitational field at the infinity. However, for the description of atomic world-where the $N_{\text {sys }} / N_{\text {Gal }} \approx 10^{-66}$-such set of equations is overkill and much simpler Lagrangian form could be derived from (59). The new Lagrangian will transfer the curved time-space description (or Lagrangian) into the standard physical description of Matter-both atomic and Gravitational-as some functions on a flat Minkowski space.

Even though the Lagrangian (59) above is written in covariant form it is-due to the fact that we derived it as a series by parameter $1 / G\left(1 / G \approx 1 / G_{\infty}=\sqrt{K_{N} \hbar / c^{3}}\right)$ and because we neglected (dropped out) the terms proportional to $1 / G^{3}$ and smaller-only correct in its first approximation with regard to $1 / G^{2} \approx 1 / G_{\infty}^{2}=$ $K_{N} \hbar / c^{3}$. For that reasons we will rewrite the Lagrangian as a series of the parameter $1 / G_{\infty}^{2}$, where all functions, marked as "bar" (e.x. $\bar{S}_{i}$ ), are defined on flat space. The system of coordinates could be chosen in such a way that the tensor $\mathbf{r}$ in the first order of approximation has a Minkowski form $r_{i j} \approx \delta_{i j}=\operatorname{diag}(1,-1,-1,-1)$ and where the vector $\mathbf{U}$ has only time coordinate $(U \approx(1,0,0,0))$. The indeces of the bar variables are raised by Minkowski me- 
tric.

For the metric $\mathbf{r}$ and geometric Christoffel symbols we get this approximation:

$$
\begin{aligned}
& r_{i j} \approx \delta_{i j}+\frac{1}{G_{\infty}^{2}} \bar{r}_{i j} ; \bar{r} \equiv \bar{r}_{i j} \delta^{i j} ; \bar{r}_{U} \equiv \bar{r}_{i j} \bar{U}^{i} \bar{U}^{j} ; \bar{r}^{i j}=\bar{r}_{m n} \delta^{i m} \delta^{j n} \\
& \gamma_{i j k} \equiv \frac{1}{2} r_{i j, k}+r_{i k, j}-r_{j k, i} \approx \frac{\bar{\gamma}_{i j k}}{G_{\infty}^{2}} \equiv \frac{\bar{r}_{i j, k}+\bar{r}_{i k, j}-\bar{r}_{j k, i}}{2 G_{\infty}^{2}}
\end{aligned}
$$

For the vector field $\mathbf{U}$ we have these relations:

$$
\begin{aligned}
& S_{i}=G_{\infty}^{2} \bar{U}_{i}+\bar{S}_{i} ; S=\sqrt{S_{i} S_{j} r^{i j}} \approx G_{\infty}^{2}+\frac{1}{2}\left(\bar{S}_{U}-\frac{\bar{r}_{U}}{2}\right) ; \bar{S}_{U} \equiv \bar{S}_{i} \bar{U}^{i} \\
& U_{i} \equiv \frac{S_{i}}{S} \approx \bar{U}_{i}+\frac{\bar{S}_{i}+\left(-\bar{S}_{U}+\frac{1}{2} \bar{r}_{U}\right) \bar{U}_{i}}{2 G_{\infty}^{2}} ; U_{i} U_{j} r^{i j} \approx 1+\frac{1}{G_{\infty}^{4}}[\cdots]
\end{aligned}
$$

The tensors $\mathbf{N}$ and $\mathbf{C}$ cannot be simply transfer to $\overline{\mathbf{N}}$ (and $\overline{\mathbf{C}}$ ) due to its "traceless" conditions $N_{i j k} r^{i j}=0$, which as we would expect will transfer to $\bar{N}_{i j k} \delta^{j k}=0$. However, the direct calculations give this expression:

$$
N_{i j k} r^{j k}=0=>\approx \bar{N}_{i j k}\left(\delta^{j k}-\frac{1}{G_{\infty}^{2}} \bar{r}^{j k}\right) \neq 0 .
$$

These difficulties could be resolved if we introduce the new variables accordingly these expressions:

$$
\begin{aligned}
& N_{i j k}=\bar{N}_{i j k}+\frac{1}{6 G_{\infty}^{2}} v_{1}\left(\delta_{j k} \bar{N}_{i m n}+\delta_{i k} \bar{N}_{j m n}+\delta_{i j} \bar{N}_{k m n}\right) \bar{r}^{m n} \\
& +\frac{1}{2 G_{\infty}^{2}} v_{2}\left(\bar{N}_{i j m} \bar{r}_{n k}+\bar{N}_{i k m} \bar{r}_{n j}+\bar{N}_{j k m} \bar{r}_{n i}\right) \delta^{m n}=> \\
& N_{i j k} r^{j k} \approx N_{i j k}\left(\delta^{j k}-\bar{r}^{j k}\right) \approx \bar{N}_{i j k} \delta^{i j k}+\frac{v_{1}+v_{2}-1}{G_{\infty}^{2}} \bar{N}_{i j k} \bar{r}^{j k}
\end{aligned}
$$

And if $v_{1}+v_{2}=1$, we get $\overline{\mathbf{N}}$ satisfies the condition $\bar{N}_{i j k} \delta^{i j}=0$.

Similarly, the transition from $\mathbf{C}$ to $\overline{\mathbf{C}}$ goes by these expressions:

$$
\begin{aligned}
& C_{i j k}=\bar{C}_{i j k}+\frac{1}{3 G_{\infty}^{2}} v_{1}^{(C)}\left(\delta_{i j} \bar{C}_{k m n}-\delta_{i k} \bar{C}_{j m n}\right) \bar{r}^{m n}+\frac{1}{G_{\infty}^{2}} v_{2}^{(C)}\left(\bar{C}_{i j m} \bar{r}_{n k}-\bar{C}_{i k m} \bar{r}_{n j}\right) \delta^{m n}=> \\
& C_{i j k} r^{i j} \approx C_{i j k}\left(\delta^{j k}-\bar{r}^{i j}\right) \approx \bar{C}_{i j k} \delta^{i j}-\frac{v_{1}^{(C)}+v_{2}^{(C)}-1}{G_{\infty}^{2}} \bar{C}_{i j k} \bar{r}^{j k}
\end{aligned}
$$

And if $v_{1}^{(C)}+v_{2}^{(C)}=1$, we get $\overline{\mathbf{C}}$ satisfies the condition $\bar{C}_{i j k} \delta^{i j}=0$.

The covariant derivatives of tensor $\mathbf{H}$ (or $\mathbf{N}$ and $\mathbf{C}$ ) transition to "bar" variables accordingly these expressions:

$$
\mathbf{H}^{\prime} \equiv \overline{\mathbf{H}}^{\prime}-\frac{1}{G_{\infty}^{2}} \overline{\mathbf{H}}^{\prime} \overline{\mathbf{r}}-\frac{1}{G_{\infty}^{2}} \overline{\mathbf{H}} \overline{\mathbf{r}}^{\prime} \quad \text { where } \quad \mathbf{H}^{\prime} \equiv H_{i j k ; l} \text { and } \overline{\mathbf{H}}^{\prime} \equiv \bar{H}_{i j k, l}
$$

It is important here to note that since the fully symmetric tensors $\mathbf{N}$ and torsion type tensor $\mathbf{C}$ are defined thru the tensor $\mathbf{P}$ with all indeces down, the transition to "bar" variables must be done on the tensor $\mathbf{N}$ and $\mathbf{C}$ (or H ) with all indeces being low. So, for example, $N_{i j k}=>\bar{N}_{i j k}$, but 
$N_{j k}^{i}=N_{m j k} r^{m i} \Rightarrow \bar{N}_{i j k} \delta^{m i}-\bar{N}_{m j k} \bar{r}_{n l} \delta^{m n} \delta^{l i}$.

Using the expressions (61) thru (66) we can easily get that the Lagrangian of the Matter $L^{(M)}$ has this linear (in terms of $\frac{1}{G_{\infty}^{2}}$ ) form:

$$
L^{(M)} \sqrt{-\operatorname{det}\left(r_{i j}\right)} \approx \bar{L}^{(M)}+\frac{\bar{J}_{i}^{(M U)} \bar{S}^{i}+\bar{F}_{i j}^{(M r)} \bar{r}^{i j}}{G_{\infty}^{2}} \text { or } L^{(M)}=\bar{L}^{(M)}+\frac{\overline{\mathbf{J}}^{(M U)} \overline{\mathbf{S}}+\overline{\mathbf{F}}^{(M r)} \overline{\mathbf{r}}}{G_{\infty}^{2}}
$$

where $\overline{\mathbf{F}}^{(M r)}$ is a symmetrical tensor and $\overline{\mathbf{J}}^{(M U)}$ is a vector, which is derived from the fact that Lagrangian $L^{(M)}$ contains terms with the unity vector $\mathbf{U}$.

With regard to the tensor $\overline{\mathbf{F}}^{(M r)}$ it is important to point out that because the metric tensor $\mathbf{r}$ comes into the tensor of Matter (M) and thus Lagrangian not only algebraically, but also in covariant derivatives and into flat-space presentations of the tensor $\overline{\mathbf{H}}$ (see expr. (66)), the symmetrical tensor $\overline{\mathbf{F}}^{(M r)}$ includes (and not equal to) the Energy-Momentum $\mathbf{T} \equiv T_{i j}$ tensor. As an exception, it's not difficult to see that in particular case of Maxwell Lagrangian-due to the fact that is contains only a low index vector, contains no unit vector $\mathbf{U}$ and contains no co-variant derivatives-the $\overline{\mathbf{J}}^{\text {(Max) }}=0$ and $\overline{\mathbf{F}}^{\text {(Max) }}$ is the tensor of energy-momentum of the Electromagnetic field. Also, it need to be mentioned that in vacuum both tensors $\overline{\mathbf{F}}^{(M r)}$ and $\overline{\mathbf{S}}^{(M S)}$ vanish.

If we consider the interaction terms of the Gravitational Lagrangian$\mathbf{S}^{\prime}\left(\mathbf{M Q}+\mathbf{Q}^{3}\right)$ and $\mathbf{R}^{\mathbf{G}}\left(\mathbf{Q}^{2}+\mathbf{M}\right)$-we can make this observation: because it comes with a factor $\frac{1}{G_{\infty}^{2}}$, the transition to the "bar" variables" are strait for ward-simply replace all sub-fields with the "bar" ones- $\mathbf{H}=>\overline{\mathbf{H}}, \mathbf{B}=>\overline{\mathbf{B}}$, $\mathbf{D}=>\overline{\mathbf{D}}, \quad \mathbf{E}=>\overline{\mathbf{E}}$ and the determinate $\sqrt{-\operatorname{det}\left(r_{i j}\right)}$ could be set to one. Also the geometric Riemann tensor $R_{i j k l}$ in these "curved space"-Matter interaction terms $\mathbf{R}^{\mathbf{G}}\left(\mathbf{M}+\mathbf{Q}^{2}\right)$ could be replace by its linear part $\left(\bar{R}_{i j k l}\right)$, which are second derivatives of metric $-\overline{\mathbf{r}}^{\prime \prime}\left(\overline{\mathbf{M}}+\overline{\mathbf{Q}}^{2}\right)$.

More care need to be taken for the derivatives of the vector $\mathbf{S}\left(\mathbf{S}^{\prime}\right)$. The covariant derivatives of $\mathbf{S}$ can be written in this form:

$$
\begin{aligned}
S_{i, j} & =S_{i, j}-\gamma_{i j}^{m} S_{m} \approx \bar{S}_{i, j}-\bar{\gamma}_{i j}^{m} \bar{U}_{m} \\
& =\bar{S}_{i, j}-\frac{1}{2}\left(\bar{r}_{m i, j}+r_{m j, i}-\bar{r}_{j k, m}\right) \bar{U}^{m} \quad \text { or } \quad \mathbf{S}^{\prime} \approx \overline{\mathbf{S}}^{\prime}+\overline{\mathbf{r}}^{\prime}
\end{aligned}
$$

The most general expression for the $\mathbf{S}^{2}$ has only 8 terms with $\sigma$-coefficient attached to each one.

$$
\begin{aligned}
L^{\left(S^{\prime}\right) \equiv} \equiv & \sum_{s=\text { inv }} \lambda_{s}^{(S)} \mathbf{S}^{2} \\
= & S_{i ; j} S_{k ; l}\left[\sigma_{1} r^{i k} r^{j l}+\sigma_{2} r^{i l} r^{j k}+\sigma_{3} r^{i j} r^{k l}\right. \\
& \left.\quad+\sigma_{4} r^{i k} U^{j} U^{l}+\sigma_{5} r^{j l} U^{i} U^{k}+\sigma_{6} r^{j k} U^{i} U^{l}+\sigma_{7} r^{i j} U^{k} U^{l}+\sigma_{8} U^{i} U^{j} U^{k} U^{l}\right] \\
\approx & \left(\bar{S}_{i, j}-\bar{\gamma}_{i j}^{s} \bar{U}_{s}\right)\left(\bar{S}_{k, l}-\bar{\gamma}_{k l}^{s} \bar{U}_{s}\right) \\
& {\left[\sigma_{1} \delta^{i k} \delta^{j l}+\sigma_{2} \delta^{i l} \delta^{j k}+\sigma_{3} \delta^{i j} \delta^{k l}\right.} \\
& \left.+\sigma_{4} \delta^{i k} \bar{U}^{j} \bar{U}^{l}+\sigma_{5} \delta^{j l} \bar{U}^{i} \bar{U}^{k}+\sigma_{6} \delta^{j k} \bar{U}^{i} \bar{U}^{l}+\sigma_{7} \delta^{i j} \bar{U}^{k} \bar{U}^{l}+\sigma_{8} \bar{U}^{i} \bar{U}^{j} \bar{U}^{k} \bar{U}^{l}\right]
\end{aligned}
$$


with $\sigma$-s being constants depending on the $\lambda$-s constants of the Lagrangian (30).

This expression contains 3 groups of terms: $\overline{\mathbf{S}}^{\prime 2}, \overline{\mathbf{S}}^{\prime} \overline{\mathbf{r}}^{\prime}$ and $\overline{\mathbf{r}}^{\prime 2}$.

$$
\bar{L}^{\left(S^{\prime}\right)} \approx \bar{L}^{\left(S^{\prime} S^{\prime}\right)}+\bar{L}^{\left(S^{\prime} r^{\prime}\right)}+\bar{L}^{\left(r^{\prime} r^{\prime}\right)}
$$

The exact expression for the $L^{\left(S^{\prime} r^{\prime}\right)}$ and $L^{\left(r^{\prime} r^{\prime}\right)}$ can be easily calculated using expr. (69) which we will do in the following section. Combining (67) and (70) together we get this expression for the $L^{\text {Grav }}$ :

$$
\begin{aligned}
\bar{L}^{(\text {Grav })} & \approx \frac{\bar{L}^{\left(S^{\prime} S^{\prime}\right)}+\bar{L}^{\left(S^{\prime} r^{\prime}\right)}+\bar{L}^{\left(r^{\prime} r^{\prime}\right)}+L^{\left(M S^{\prime}\right)}+L^{(M r)}}{G_{\infty}^{2}} \\
& \text { or } \approx \frac{\overline{\mathbf{S}}^{\prime 2}+\overline{\mathbf{S}}^{\prime} \overline{\mathbf{r}}^{\prime}+\overline{\mathbf{r}}^{\prime 2}+\mathbf{J}^{(S)} \overline{\mathbf{S}}+\mathbf{J}^{(r)} \overline{\mathbf{r}}}{G_{\infty}^{2}}
\end{aligned}
$$

The linearized expression for the action integral for just Einstein term $\left(G^{2} R\right)$ could be obtained in this manner:

$$
\begin{aligned}
& S^{(R)}=\int \mathrm{d}^{4} x G^{2} R \sqrt{-\operatorname{det}\left(r_{i j}\right)}=\int \mathrm{d}^{4} x G^{2} R_{i j k l} r^{i k} r^{j l} \sqrt{-\operatorname{det}\left(r_{i j}\right)} \\
& =\int \mathrm{d}^{4} x \sqrt{-\operatorname{det}\left(r_{i j}\right)} \sqrt{S_{m} S_{n} r^{n m}} \frac{r_{i l, j k}+r_{j k, i l}-r_{i k, j l}-r_{j l, i k}}{2} r^{i k} r^{j l} \\
& +\int \mathrm{d}^{4} x \sqrt{-\operatorname{det}\left(r_{i j}\right)} \sqrt{S_{m} S_{n} r^{n m}}\left(\gamma_{k m}^{k} \gamma_{j l}^{m}-\gamma_{l m}^{k} \gamma_{j k}^{m}\right) r^{j l} \\
& \approx \int \mathrm{d}^{4} x\left(1+\frac{\bar{r}}{2 G_{\infty}^{2}}\right)\left(1+\frac{\bar{S}_{U}-\frac{\bar{r}_{U}}{2}}{G_{\infty}^{2}}\right) \bar{R}_{i j k l}\left(\delta^{i k}-\frac{\bar{r}^{i k}}{G_{\infty}^{2}}\right)\left(\delta^{j l}-\frac{\bar{r}^{j l}}{G_{\infty}^{2}}\right) \\
& +\int \mathrm{d}^{4} x \frac{1}{G_{\infty}^{2}}\left(\bar{\gamma}_{k m}^{k} \bar{\gamma}_{j l}^{m}-\bar{\gamma}_{l m}^{k} \bar{\gamma}_{j k}^{m}\right) \delta^{j l} \\
& \approx \underline{\int \mathrm{d}^{4} x \bar{R}}+\int \mathrm{d}^{4} x \frac{1}{G_{\infty}^{2}}\left[\left(\bar{S}_{U}-\frac{\bar{r}_{U}}{2}\right) \bar{R}-2 \bar{R}_{i j} \bar{r}^{i j}+\frac{1}{2} \bar{R} \bar{r}\right] \\
& +\underline{+\int \mathrm{d}^{4} x \frac{1}{G_{\infty}^{2}}\left[\left(\bar{\gamma}_{k m}^{k} \bar{\gamma}_{j l}^{m}-\bar{\gamma}_{l m}^{k} \bar{\gamma}_{j k}^{m}\right) \delta^{j l}\right]}
\end{aligned}
$$

where;

$$
\begin{aligned}
\bar{R}_{i j k l} & =\frac{1}{2}\left(\bar{r}_{i l, j k}+\bar{r}_{j k, i l}-\bar{r}_{i k, j l}-\bar{r}_{j l, i k}\right) \bar{R}_{i j}=\bar{R}_{i j k l} \delta^{i k} ; \quad \bar{R}=\bar{R}_{j l} \delta^{j l} \\
\bar{S}_{U} & =\bar{S}_{i} \bar{U}^{i} ; \bar{r}_{U}=\bar{r}_{i j} \bar{U}^{i} \bar{U}^{j} ; \bar{r}=\bar{r}_{i j} \delta^{i j} \bar{r}^{i j}=\bar{r}_{m n} \delta^{m i} \delta^{n j}
\end{aligned}
$$

Both underlined terms vanish thru the means of partial integrations, so the final form for the action integral for the Einstein terms has this form:

$$
S^{(R)} \approx \int \mathrm{d}^{4} x \bar{L}^{(R)} ; \text { where } \bar{L}^{(R)}=\frac{1}{G_{\infty}^{2}}\left[\left(\bar{S}_{U}-\frac{\bar{r}_{U}}{2}\right) \bar{R}-2 \bar{R}_{i j} \bar{r}^{i j}+\frac{1}{2} \bar{R} \bar{r}\right]
$$

We can see from this that Einstein term $G^{2} R$ is of the same form-in terms of order of magnitude $\left(1 / G_{\infty}^{2}\right)$ and in terms of variables $\left(\mathbf{r}^{\prime \prime}\right.$ and $\left.\mathbf{r}^{\prime} \mathbf{S}^{\prime}\right)$ as the linearized form of the Lagrangian $L^{\left(S^{\prime}\right)}$ in expr. (71).

Combining all sub-Lagrangians together-expression (71) and (73)-we can write the final expression for the linear, with respect the factor $\frac{1}{G_{\infty}^{2}}$ approximation of the Lagrangian of Total-Matter: 


$$
\begin{aligned}
& \begin{array}{l}
S=\int \sqrt{-r} \mathrm{~d}^{4} x L \approx \int \mathrm{d}^{4} x \bar{L} ; \quad \bar{L}=\bar{L}^{M)}+\frac{\bar{L}^{(\text {Grav })}}{G_{\infty}^{2}} \\
=\bar{L}^{(M)}+\frac{\bar{L}^{S S^{\prime}}+\bar{L}^{S r^{\prime}}+\bar{L}^{r^{\prime} r^{\prime}}+\bar{L}^{M S}+\bar{L}^{M r}}{G_{\infty}^{2}}
\end{array} \\
& \text { where } \bar{L}^{(M)}=\bar{L}^{(M)}\left(\mathbf{H}, \mathbf{B}, \mathbf{D}, \mathbf{E}^{*}\right) ; \bar{L}^{\left(S^{\prime} S^{\prime}\right)}=\overline{\mathbf{S}}^{\prime} \overline{\mathbf{S}}^{\prime} ; \quad L^{\left(S^{\prime} r^{\prime}\right)}=\overline{\mathbf{S}}^{\prime} \overline{\mathbf{r}}^{\prime} ; L^{\left(r^{r^{\prime}}\right)}=\overline{\mathbf{r}}^{\prime} \overline{\mathbf{r}}^{\prime} \\
& \bar{L}^{M S}=\bar{J}_{i}^{(S)}\left(\mathbf{H}, \mathbf{B}, \mathbf{D}, \mathbf{E}^{*}\right) \bar{S}^{i} \bar{L}^{M r}=\bar{F}_{i j}^{(r)}\left(\mathbf{H}, \mathbf{B}, \mathbf{D}, \mathbf{E}^{*}\right) \bar{r}^{i j}
\end{aligned}
$$

The Lagrangian above represents exactly the physical phenomenon that we postulated at the beginning of this paper. The equations of motion for Matter, which includes mass-particle (tensor $\mathbf{H}$ ), electromagnetic field (vector field E) as well as two other vector fields ( $\mathbf{B}$ and $\mathbf{D}$ ) do not depend on the metric $\mathbf{r}$ and/or grav-vector $\mathbf{S}$. Their description is well determined by set of equations written in flat Minkowski space.

The description of metric $\mathbf{r}$ and Grav-vector $\mathbf{S}$ could be presented as a correction $\overline{\mathbf{r}}$ and $\overline{\mathbf{S}}$ to a constant (flat) space and constant grav-field. The equations for corrections has a traditional flat space field description with each one of them having a source defined by mass-matter $\mathbf{H}$ and vector fields $\mathbf{B}$, electromagnetic field $\mathbf{E}$ and $\mathbf{D}$.

The terms grouped in the Lagrangian $L^{\left(S^{\prime} r^{\prime}\right)}$ represent a coupling (inter-dependence) of the metric $\overline{\mathbf{r}}$ and $\overline{\mathbf{S}}$. In the "Gravitation" section it will be shown that these equations could be decoupled using a "gage" requirements for the metric $\mathbf{r}$, which is defined up to 4 functions associated with arbitrarily system of coordinates. And by doing so we will effectively separate out a "chosen" or "rest" system of coordinate from all inertial system of coordinates.

We will address in greater details the equations of motion for each variable in their corresponding sections-"Gravitation" for $\mathbf{S}$ and $\mathbf{r}$, "Electromagnetic and Other Fields" for B, D, E and "Mass-Matter (Elementary Particles)" for H.

Before we proceed writing the equations for each of these fields we would like make few general comments:

1) The Lagrangian (74) is a in effect the Einstein's Lagrangian where Gravvector $\mathbf{S}$ is a dark matter and the magnitude of the Grav-vector $\mathbf{S}\left(S=G^{2}\right)$ serving as gravitational constant.

It worth pointing out that the form of the Lagrangian of the Total-Matter is identical to the standard General Relativity written in slightly different manner, where we multiply the Lagrangian of General relativity by a constant $\frac{c^{4}}{K_{N} \hbar c}$, express the Lagrangian and thus the Energy-Momentum tensor in units $\hbar c$ and write approximation for metric tensor in a form $r_{i j}=\delta_{i j}+\frac{K_{N} \hbar c}{c^{4}} \bar{r}_{i j}$ :

$$
\begin{aligned}
L^{(\mathrm{GenRel})} & =\frac{c^{3}}{K_{N} \hbar}\left[\frac{K_{N} \hbar c}{c^{4}} L^{(M)}+R\right]=L^{(M)}+\frac{c^{3}}{K_{N} \hbar} R \\
& \approx L^{(M)}+\frac{1}{G_{\infty}^{2}}\left[\bar{T}^{i j} \bar{r}_{i j}-2 \bar{R}^{i j} \bar{r}_{i j}+\frac{1}{2} \bar{R} \bar{r}_{i j} \delta^{i j}\right] \text { where } G_{\infty}^{2} \equiv \frac{c^{3}}{K_{N} \hbar} .
\end{aligned}
$$


2) According to this theoretical derivations using Affine Unification this Lagrangian is a linear approximation for a system of masses (like our Solar system and smaller), where Grav-field created by the system is a small addition to the already existing Grav-field created by the masses outside of our system-such as our Galaxy, or group of Galaxies, or the entire Universe. It is for that reasons, the considerations of Universe models taken Einstein equations as a starting point (with or without Dark Matter) might not be a justified approach. We will address the issue again in the section "Cosmology".

3) We derive this Lagrangian from the Unified Affine description by imposing certain requirements on parameters $\lambda_{s}$-see expr. (30) - in order for this theory to be non contradicting to the common experimental facts and to common sense. But as we continue investigating the equations of motions for each unknown variable $(\mathbf{H}, \mathbf{S}, \mathbf{B}, \mathbf{D}, \mathbf{E}, \mathbf{r})$ we might (and more likely will) need to impose more requirements on $\lambda_{s}$. What is important here is that these requirements simply outlaw some terms in the final Lagrangian. However, we are totally controlled by the "affine derivation" to the degree what type of terms is allowed to be present as a part of the Lagrangian.

4) Regarding vanishing of "unwanted" by means of setting some requirements on the $\lambda$-constants we would like to point out to the fact that there is of course much easier way of removing unwanted terms in the general Lagrangian. In stead doing of the calculation and finding the right $\lambda$-s, thru solving all the equation-conditions that one places on $\lambda$-s, we can simple postulate that this term in the Lagrangian of the Total-Matter is zero. For example, in stead of searching for $\lambda$-s such that $G^{2}\left\{\sum_{s=\text { allinv }} \lambda_{s}\left[\mathbf{I}^{2}\left(\mathbf{Q}^{2}+\mathbf{M}\right)\right]\right\}=0$, we could take a Lagrangian almost at will and then place a requirement that the terms of the Lagrangian proportional to $G^{2}$ is zero. Express in the "Lagrange coefficients" this would effectively add one more unknown function (call it $\Lambda^{\left(G^{2}\right)}$ ) as a dynamic variable. Between removing all terms proportional to $G^{n} \quad(n=4,3,2,1$, and -1 ) and some unacceptable terms inside the Lagrangian $\mathbf{M}$ for the Matter and Lagrangian for the gravitational field $\overline{\mathbf{S}}$ and $\overline{\mathbf{r}}$ there will be somewhere around dozen or so such new unknown functions.

The problem with such approach is its universality. This method could be apply to any Lagrangian and thus totally leaving unanswered the question how this Lagrangian actually looks. Another words, we have no idea which Lagrangian (out of thousand or so) we should really choose. The approach that we took in this paper (defining the $\lambda$-s) is much more complex precisely because it actually defines (selects) the right Lagrangian. However, if we outrun number of available constants or get a contradiction in the constants' values, it is much more realistic to envision a mid-point situation that most requirements (such for example that involve the vector fields $\mathbf{B}, \mathbf{D}$ and $\mathbf{E}$ ) for vanishing "unwanted" terms can be met thru the form of Lagrangian $(\lambda-s)$ and thru the form of Total-Matter $(\mu-\mathrm{s})$. The remaining non-vanishing terms would be related to only one group of functions ( $\mathbf{H}$ or $\mathbf{N}$ and $\mathbf{C}$ ) could be vanished by introduction of Lagrange-coefficients. 


\section{Equations for Gravitational Field and Metric}

This section deals with equations of motion for the square-vector $\mathbf{S}$ and the metric $\mathbf{r}$ Let us again write the flat-space Lagrangian of the Total-Matter $\bar{L}$ :

$$
\begin{aligned}
S \approx \int \bar{L} \mathrm{~d}^{4} x \quad & =\bar{L}^{(M)}+\frac{\bar{L}^{\mathrm{Grav}}}{G_{\infty}^{2}}=\bar{L}^{(M)}+\frac{\bar{L}^{\left(S^{\prime} S^{\prime}\right)}+\bar{L}^{\left(S^{\prime} r^{\prime}\right)}+\bar{L}^{\left(r^{\prime} r^{\prime}\right)}+\bar{L}^{(M S)}+\bar{L}^{(M r)}}{G_{\infty}^{2}} \\
\text { where } & \bar{L}^{(M)}=\bar{L}^{(M)}(\mathbf{H}, \mathbf{B}, \mathbf{D}, \mathbf{E}) ; \bar{L}^{(M S)}=\bar{J}_{i}^{(S)}(\mathbf{H}, \mathbf{B}, \mathbf{D}, \mathbf{E}) \bar{S}^{i} ; \\
& \bar{L}^{M r}=\bar{J}_{i j}^{(r)}(\mathbf{H}, \mathbf{B}, \mathbf{D}, \mathbf{E}) \bar{r}^{i j} \\
& \bar{L}^{\left(S^{\prime} S^{\prime}\right)}=\sum_{\text {allinv }} \sigma^{(S)} \overline{\mathbf{S}}^{\prime} \overline{\mathbf{S}}^{\prime} ; \bar{L}^{\left(S^{\prime} r^{\prime}\right)}=\sum_{\text {allinv }} \sigma^{(S r)} \overline{\mathbf{S}}^{\prime} \overline{\mathbf{r}}^{\prime} ; \bar{L}^{(r)}=\sum_{\text {allinv }} \sigma^{(r)} \overline{\mathbf{r}}^{\prime} \overline{\mathbf{r}}^{\prime} ;
\end{aligned}
$$

where $\sigma^{(S)}$ are set of constants linearly depending on the $\sigma$-s constants of expr. (69), which by themselves are functions of the $\lambda$-constants of the Lagrangian, and where $\bar{L}^{\left(S^{\prime} S^{\prime}\right)},\left(\bar{L}^{\left(S^{\prime} r^{\prime}\right)}, \bar{L}^{\left(r^{\prime} r^{\prime}\right)}\right)$ are the terms that all came from decomposition (writing) of covariant derivatives $\mathbf{S}^{2}$ of the Lagrangian-see expr. (59) - on three groups: a) square of partial derivatives of $\overline{\mathbf{S}}\left(\overline{\mathbf{S}}^{\prime}\right)$; b) square if partial derivatives of $\overline{\mathbf{r}}\left(\overline{\mathbf{r}}^{\prime}\right)$ and $c$ ) the product of $\overline{\mathbf{S}}^{\prime} \overline{\mathbf{r}}^{\prime}$.

The first group with some simple manipulations (like partial integration) could be written in this form:

$$
\begin{aligned}
\bar{L}^{(S)}=\bar{S}_{i, j} \bar{S}_{k, l}[ & \sigma_{1} \delta^{i k} \delta^{j l}+\left(\sigma_{2}+\sigma_{3}\right) \delta^{i j} \delta^{k l}+\sigma_{4} U^{k} U^{l} \delta^{i k} \\
& \left.+\sigma_{5} \delta^{j l} \bar{U}^{i} \bar{U}^{k}+\left(\sigma_{6}+\sigma_{7}\right) \delta^{i j} \bar{U}^{k} \bar{U}^{l}+\sigma_{8} \bar{U}^{i} \bar{U}^{j} \bar{U}^{k} \bar{U}^{l}\right] .
\end{aligned}
$$

Since in Minkowski space the unit vector $\bar{U}_{i}=(1,0,0,0)$, the equations above could be written in somewhat more explicit (if not simpler) form where we use "0" as time coordinate.

$$
\begin{aligned}
\bar{L}^{(S)}= & \sigma_{1} \bar{S}_{i, j} \bar{S}_{k, l} \delta^{i k} \delta^{j l}+\left(\sigma_{2}+\sigma_{3}\right) \bar{S}_{i, j} \bar{S}_{k, l} \delta^{i j} \delta^{k l}+\sigma_{4} \bar{S}_{i, 0} \bar{S}_{k, 0} \delta^{i k} \\
& +\sigma_{5} \bar{S}_{0, j} \bar{S}_{0, l} \delta^{j l}+\left(\sigma_{6}+\sigma_{7}\right) \bar{S}_{i, j} \bar{S}_{0,0} \delta^{i j}+\sigma_{8}\left(\bar{S}_{0,0}\right)^{2}
\end{aligned}
$$

Although for derivation of equations for $\overline{\mathbf{S}}_{i}$ by variation of $\bar{S}$ is better to use expr. (76) and switch to Minkowski coordinate at the very end. Thus variation of the term $\sigma_{8}\left(\bar{S}_{0,0}\right)^{2}$ we get $-2 \sigma_{8} \bar{S}_{j, k l} \bar{U}_{i} \bar{U}^{j} \bar{U}^{k} \bar{U}^{l}=-2 \sigma_{8} \bar{S}_{0,00} \bar{U}_{i}$. The same form of writing (zero instead of time coordinate) can be applied to the others sub-Lagrangians of the $L^{\mathrm{Grav}}$. For, example the $\left(\sigma_{6}+\sigma_{7}\right) S_{i, j} r^{i j} S_{k ; l} U^{k} U^{l}$ term can be written as this:

$$
\begin{aligned}
S_{i ; j} r^{i j} S_{k ; l} U^{k} U^{l} \approx & \left(\bar{S}_{i, j}-\bar{\gamma}_{i j}^{s} \bar{U}_{s}\right) \delta^{i j}\left(\bar{S}_{k, l}-\bar{\gamma}_{k l}^{s} \bar{U}_{s}\right) \bar{U}^{k} \bar{U}^{l} \\
= & {\left[\bar{S}_{i, j}-\frac{1}{2}\left(\bar{r}_{0 i, j}+\bar{r}_{0 j, i}-\bar{r}_{i j, 0}\right)\right] \delta^{i j}\left(\bar{S}_{0,0}-\frac{1}{2} \bar{r}_{00,0}\right) } \\
= & {\left[\bar{S}_{i, j} \delta^{i j} \bar{S}_{0,0}\right]^{\left(S^{\prime} S^{\prime}\right)}+\left[-\frac{1}{2} \bar{S}_{i, j} \delta^{i j} \bar{r}_{0,0}-\frac{1}{2} \bar{S}_{0,0}\left(2 \bar{r}_{0, j} \delta^{i j}-\bar{r}_{i j, 0}\right)\right]^{\left(S^{\prime}{ }^{\prime}\right)} } \\
& +\left[\frac{1}{4}\left(2 \bar{r}_{0 i, j} \delta^{i j}-\bar{r}_{i j, 0}\right) \bar{r}_{0,0}\right]^{\left(r^{\prime} r^{\prime}\right)}
\end{aligned}
$$

where by square bracket with appropriate label (in parentheses) we indicated to which sub-Lagrangian which terms belong. The "interaction" part of the La- 
grangian- $\overline{\overline{\mathbf{S}}^{\prime}} \overline{\mathbf{r}}^{\prime}$ terms-could also be written (thru partial integrations) in the form $\overline{\mathbf{S}} \overline{\mathbf{r}}^{\prime \prime}$. Using this form of writing the Gravitational sub-Lagrangians $\bar{L}^{\left(S^{\prime}\right)}$, $\bar{L}^{\left(S^{\prime} r^{\prime}\right)}$ and $\bar{L}^{\left(r^{\prime} r^{\prime}\right)}$ can be written in this form:

$$
\begin{aligned}
\bar{L}^{\left(S^{\prime} S^{\prime}\right)}= & \sigma_{1} \bar{S}_{i, j} \bar{S}_{k, l} \delta^{i k} \delta^{j l}+\left(\sigma_{2}+\sigma_{3}\right) \bar{S}_{i, j} \bar{S}_{k, l} \delta^{i j} \delta^{k l}+\sigma_{4} \bar{S}_{i, 0} \bar{S}_{k, 0} \delta^{i k} \\
& +\sigma_{5} \bar{S}_{0, j} \bar{S}_{0, l} \delta^{j l}+\left(\sigma_{6}+\sigma_{7}\right) \bar{S}_{i, j} \bar{S}_{0,0} \delta^{i j}+\sigma_{8}\left(\bar{S}_{0,0}\right)^{2} \\
\bar{L}^{\left(S^{\prime}{ }^{\prime}\right)}=\bar{S}^{i}[ & \left(\sigma_{1}+\sigma_{2}\right) \bar{r}_{0 i, k l} \delta^{k l}+\left(\sigma_{1}+\sigma_{2}+2 \sigma_{3}\right) \bar{r}_{0 k, l i} \delta^{k l} \\
- & \left(\sigma_{1}+\sigma_{2}\right) \bar{r}_{i k, l 0} \delta^{k l}-\left(\sigma_{3}\right) \bar{r}_{0 i}+\left(\sigma_{7}\right) \bar{r}_{0 i, 00}+\left(\sigma_{4}+\frac{\sigma_{6}+\sigma_{7}}{2}\right) \bar{r}_{00,0 i} \\
+ & U_{i}\left(\sigma_{5}+\frac{\sigma_{6}}{2}\right) \bar{r}_{00, k l} \bar{\delta}^{k l} \\
- & \left.\frac{1}{2} \bar{U}_{i}\left(\sigma_{7}\right) \bar{r}_{00}+\bar{U}_{i} \sigma_{8} r_{00,00}+\sigma_{0} \bar{U}_{i}\left(\bar{r}_{k l, m n} \delta^{k m} \delta^{\mathrm{ln}}-\bar{r}_{, k l} \delta^{k l}\right)\right] \\
L^{r^{\prime} r^{\prime}}= & -\frac{\sigma_{1}+\sigma_{2}}{4}\left(2 \bar{r}_{0 i} \bar{r}_{0 j, m n} \delta^{i j} \delta^{m n}+\bar{r}_{i j, 0} \bar{r}_{k l, 0} \delta^{i k} \delta^{j l}\right. \\
& \left.+2 \bar{r}_{0 i} \bar{r}_{0 m, n j} \delta^{i j} \delta^{m n}-4 \bar{r}_{0 i} \bar{r}_{j m, n 0} \delta^{i j} \delta^{m n}\right) \\
& +\frac{\sigma_{3}}{4}\left(4 \bar{r}_{0 i, j} \bar{r}_{0 m, n} \delta^{i j} \delta^{m n}-2 \bar{r}_{0 i, j} \bar{r}_{00} \delta^{i j}+\bar{r}_{, 0} \bar{r}_{0}\right) \\
& +\frac{\sigma_{4}+\sigma_{5}+\sigma_{6}}{4} \bar{r}_{00, i} \bar{r}_{00, j} \delta^{i j}+\frac{\sigma_{7}}{4}\left(\bar{r}_{0 i, j} \bar{r}_{00,0} \delta^{i j}+\bar{r}_{, 0} \bar{r}_{00,0}\right) \\
& +\frac{\sigma_{8}}{4}\left(\bar{r}_{00,00}\right)^{2} \sigma_{0} \bar{r} \bar{R}-\sigma_{0} \bar{r}_{00} \bar{R}+\sigma_{0} \bar{r}_{i j} \bar{R}_{k l} \delta^{i k} \delta^{j l}
\end{aligned}
$$$$
\text { where } \bar{R}_{j l}=\frac{1}{2}\left[\left(\bar{r}_{i j, k l}+\bar{r}_{i l, k j}\right) \delta^{i k}-r_{j l}-r_{j l, i k} \delta^{i k}\right] ; \quad \bar{R}=R_{j l} \delta^{j l}
$$

In the expr above we for the sake of uniformity replaced $\lambda^{(R)}$ with $\sigma_{0}: \sigma_{0} \equiv \lambda^{(R)}$. The above Lagrangian is a quadratic with respect to the first derivative of either metric $\overline{\mathbf{r}}$ or grav-field $\overline{\mathbf{S}}$, which will produce linear system of equations for each variable. However due to their interactions-Lagrangian $L^{\left(S^{\prime}{ }^{\prime}\right)}$ - the equations of motion will have "interactive" form, meaning that equations for grav-vector $\overline{\mathbf{S}}$ will include metric $\overline{\mathbf{r}}$ and vice-versa. In symbolic form these equations could be written as:

$$
\overline{\mathbf{S}}^{\prime \prime}=\overline{\mathbf{r}}^{\prime \prime}+\mathbf{J}^{(S M)} ; \overline{\mathbf{r}}^{\prime \prime}=\overline{\mathbf{S}}^{\prime \prime}+\mathbf{J}^{(r M)} .
$$

This "interaction" could be removed is we request that metric tensor $\overline{\mathbf{r}}$ satisfies some conditions-the gage. It is not difficult to see that $L^{\left(S^{\prime} r^{\prime}\right)}$ contains 12 terms:

$$
\begin{aligned}
& r_{0 i, k l} \delta^{k l} \quad \underline{r_{0 k, l i} \delta^{k l}} \underline{r_{i k, l 0} \delta^{k l}} r_{, 0 i} r_{0 i, 00} \quad r_{00,0 i} \\
& U_{i} r_{00, k l} \delta^{k l} \underline{U_{i} r_{0 k, l 0} \delta^{k l}} U_{i} r_{00} \quad U_{i} r_{00,00} \underline{U_{i} r_{m j, k l} \delta^{m k} \delta^{j l}} U_{i, k l} r \delta^{k l}
\end{aligned}
$$

The number of terms can be reduced to 8 , if we consider a "gage" procedure, which comes form the following considerations. Variation of the gravitational Lagrangian $\bar{L}^{\text {grav }}$ (expr. (74)) by $\bar{r}_{i j}$ produce a sets of 10 equations for space metric $\overline{\mathbf{r}}$ in Minkowski space. However this system has 4 more functions than needed. Metric has only 6 independent functions as in equations above have all 
10. This can be fixed by imposing 4 additional conditions on the metric $\overline{\mathbf{r}}$ functions-so call "gage"-which takes a form of first order partial derivatives of the metric tensor $\overline{\mathbf{r}}$. In Landau [36] for example the gage has this form $\bar{r}_{i k, m} \delta^{k m}=0$. This gage condition reflects the fact that the Minkowski space-as asymptotic at infinity-is not uniquely defined, and the linear equations of "gage" removes this uncertainty.

Imposing such a gage we select one particular system of coordinates (or one group of systems of coordinates-like inertial one) as prefer one, compared to all others. One would hope that such selection is governed by a some general physical principle-like, for example, a system in which the laws of conservation have simple form. In our case we choose the gage in order to simplify the expr. (76) for the gravitational Lagrangian, which could be phrased in the physical manner: "the system of coordinates where Grav-vector and metric do not interact".

The most general form linear first order expression for the gage has this expression:

$$
\bar{r}_{i k, m} \delta^{k m}=\alpha_{1} \bar{r}_{, i}+\alpha_{2} \bar{r}_{0 i, 0}+\alpha_{3} \bar{r}_{00, i}+\alpha_{4} \bar{r}_{00,0} U_{i}+\alpha_{5} \bar{r}_{, 0} U_{i} \text { where } \bar{r}=\bar{r}_{i j} \delta^{i j} .
$$

If we apply "gage" relation to the underlined terms in expr. (84) we will reduce $L^{\left(S^{\prime} r^{\prime}\right)}$ to only 8 terms:

$$
\bar{S}^{i} \bar{r}_{0 i, k l} \delta^{k l} \bar{S}^{i} \bar{r}_{, 0 i} \quad \bar{S}^{i} \bar{r}_{0 i, 00} \quad \bar{S}^{i} \bar{r}_{00,0 i} \bar{S}_{0} \bar{r}_{00, k l} \delta^{k l} \quad \bar{S}_{0} \bar{r}_{, 00} \bar{S}_{0} \bar{r}_{00,00} \quad S_{0} \bar{r} .
$$

This "interaction" terms could be vanished, if we add to the 5 gage $\alpha$-constants 3 more constants $\left(\beta_{1}, \beta_{2}, \beta_{3}\right)$ associated with transitioning to new ("shifted") $\overline{\bar{G}}_{i}$ variable accordingly to the following linear expressions:

$$
\bar{G}_{i}=\overline{\bar{G}}_{i}+\beta_{1} \bar{r}_{0 i}+\beta_{2} \bar{r}_{00} \bar{U}_{i}+\beta_{3} \bar{r} \bar{U}_{i} \text { where } \bar{r}=\bar{r}_{i j} \delta^{i j} .
$$

Applying the gage (85) and the "shift" (87) we will transfer the expr. (81) into a expression where $L^{S^{\prime} r^{\prime}}$ has this form:

$$
\begin{aligned}
L^{\left(S^{\prime} r^{\prime}\right)}= & \overline{\bar{S}}{ }^{i} \bar{r}_{0 i, k m} \delta^{k m}\left\{\left(\sigma_{1}+\sigma_{2}+2 \beta_{1} \sigma_{1}\right)\right\} \\
+ & \overline{\bar{S}}^{i} \bar{r}_{, 0 i}\left\{2 \beta_{2}\left(\sigma_{2}-\left(\sigma_{3}\right)+\sigma_{3}\right)+\beta_{2}\left(\sigma_{5}+\sigma_{7}\right)+\left(\alpha_{1}+\alpha_{4}\right)\right. \\
& +\overline{\bar{S}}^{i} \bar{r}_{0 i, 00}\left\{\left(\sigma_{1}+\sigma_{2}+\sigma_{3}+2 \beta_{1} \sigma_{2}+2 \beta_{1} \sigma_{3}\right)-\alpha_{1}\left(\sigma_{1}+\sigma_{2}\right)\right\} \\
+ & \overline{\bar{S}}^{i} \bar{r}_{00,0 i}\left\{\sigma _ { 2 } \left(\sigma_{4}+\frac{\sigma_{6}+\sigma_{7}}{2}+2 \beta_{3}\left(\sigma_{2}+\sigma_{3}\right)+\left(\beta_{1}+\beta_{3}\right)\left(\sigma_{6}+\sigma_{7}\right)\right.\right.
\end{aligned}
$$

Demanding that $L^{S^{\prime} r^{\prime}}$ vanishes-each figure bracket should be zero-pro- 
duces 8 equations for 8 unknown constants $\left(\alpha_{1}\right.$ thru $\alpha_{5}$ and $\left.\beta_{1}, \beta_{2}, \beta_{3}\right)$, which yield this solutions for the gage $\alpha$ 's and for the "shift" $\beta$ 's thru the constants $\sigma$ 's. From eq. for $\bar{S}^{i} \bar{r}_{0 i, k m} \delta^{k m}$ (line 1) we get explicit value for the $\beta_{1}$. From eq. for the $\bar{S}^{i} \bar{r}_{0 i, 00}$ (line 4) we get an explicit value for $\alpha_{2}$. The other 6 equations splits on 2 groups of 3 each-one for $\beta_{2}, \alpha_{1}, \alpha_{4}$ and the other for $\beta_{3}, \alpha_{3}, \alpha_{4}$

$$
\begin{aligned}
& \beta_{1}=\frac{\sigma_{1}+\sigma_{2}}{2 \sigma_{1}} \quad \beta_{2}=0 \\
& \beta_{3}=-\frac{\sigma_{4}\left(\sigma_{1}+\sigma_{2}\right)+\sigma_{1}\left(\sigma_{5}+\sigma_{6} / 2\right)}{2\left(\sigma_{1}+\sigma_{4}\right) \sigma_{1}} \quad \alpha_{2}=\frac{\sigma_{1} \sigma_{7}+\sigma_{5}\left(\sigma_{1}+\sigma_{2}\right)}{\sigma_{1}\left(\sigma_{1}+\sigma_{2}\right)} \\
& \left\{-\sigma_{3}+2 \beta_{2}\left(\sigma_{2}+\sigma_{3}\right)+\beta_{2}\left(\sigma_{5}+\sigma_{7}\right)+\left(\alpha_{1}+\alpha_{4}\right)\left(\sigma_{1}+\sigma_{2}+\sigma_{3}+2 \beta_{1} \sigma_{2}+2 \beta_{1} \sigma_{3}\right)\right. \\
& \left.-\alpha_{1}\left(\sigma_{1}+\sigma_{2}\right)\right\}=0 \\
& \left\{\beta_{2}\left(2 \sigma_{5}+\sigma_{6}+\sigma_{7}+2 \sigma_{8}\right)+\beta_{1}\left(\left(\sigma_{6}+\sigma_{7}\right)\left(\alpha_{1}+\alpha_{4}\right)-\frac{\sigma_{7}}{2}-\left(\sigma_{1}+\sigma_{2}\right)\right)\right\}=0 \\
& \left\{\sigma_{4}+\frac{\sigma_{6}+\sigma_{7}}{2}+2 \beta_{3}\left(\sigma_{2}+\sigma_{3}\right)+\left(\beta_{1}+\beta_{3}\right)\left(\sigma_{6}+\sigma_{7}\right)\right. \\
& \left.+\left(\alpha_{2}+\alpha_{3}+\alpha_{5}\right)\left(\sigma_{1}+\sigma_{2}+\sigma_{3}+2 \beta_{1} \sigma_{2}+2 \beta_{1} \sigma_{3}\right)-\alpha_{3}\left(\sigma_{1}+\sigma_{2}\right)\right\}=0 \\
& \left\{\sigma_{8}+\beta_{3}\left(2 \sigma_{5}+\sigma_{6}+\sigma_{7}+2 \sigma_{8}\right)+2 \beta_{1} \sigma_{8}-\alpha_{5}\left(\sigma_{1}+\sigma_{2}\right)+\beta_{1}\left(\sigma_{6}+\sigma_{7}\right)\left(\alpha_{2}+\alpha_{3}+\alpha_{5}\right)\right\}=0
\end{aligned}
$$

What important here is that these constants $\alpha_{1}$ thru $\alpha_{5}$ and $\beta_{1}$ thru $\beta_{3}$ are uniquely defined by the constants $\sigma$-s of the Lagrangian $L^{S}$. After removing the interaction Lagrangian $L^{S^{\prime} r^{\prime}}$ of metric $\overline{\mathbf{r}}$ and grav-field $\overline{\mathbf{S}}$ the gravitational Lagrangian can be written as:

$$
L^{\text {grav }}=\left[L^{S}\left(\overline{\overline{\mathbf{S}}} \overline{\overline{\mathbf{S}}}^{\prime}\right)+\bar{J}_{i j}^{(S)} \overline{\bar{S}}^{i}\right]+\left[\bar{L}^{r}\left(\overline{\mathbf{r}}^{\prime} \overline{\mathbf{r}}^{\prime}\right)+\overline{\bar{F}}_{i j}^{(r)} \bar{r}^{i j}+\mathbf{V}^{i} \overline{\mathbf{r}}_{i}^{\prime}\right]
$$

The "double dash" in the $\overline{\bar{F}}_{i j}^{(r)}$ reflects the fact that $\bar{F}_{i j}^{(r)}$ get additional terms due to the transition from $\overline{\mathbf{S}}$ to $\overline{\overline{\mathbf{S}}}$ accordingly. The $\mathbf{V}^{i} \overline{\mathbf{r}}_{\mathbf{i}}^{\prime}$ terms represent the gage-see expr. (85). The equations of motion for the Grav-vector $\overline{\overline{\mathbf{S}}}$ and $\overline{\mathbf{r}}$ are "standard" non-interactive vector equations with its source $\overline{\mathbf{J}}^{(S)}$ and "Einstein-like" equations for the metric field $\overline{\mathbf{r}}$ with its source $\overline{\overline{\mathbf{F}}}^{(r)}$.

$$
\overline{\overline{\mathbf{S}}}^{\prime \prime}=\overline{\mathbf{J}}^{(S)} ; \overline{\mathbf{r}}^{\prime \prime}=\overline{\overline{\mathbf{F}}}^{(r)} ; \overline{\mathbf{r}}^{\prime}=0 \text { (gage equations) }
$$

For the gravitational vector $\overline{\overline{\mathbf{S}}}$ we have:

$$
\begin{aligned}
& \sigma_{1} \overline{\bar{S}}_{i, k m} \delta^{k m}+\left(\sigma_{2}+\sigma_{3}\right) \overline{\bar{S}}_{k, m i} \delta^{k m}+\sigma_{4} \overline{\bar{S}}_{i, 00}+\sigma_{5} \overline{\bar{S}}_{0, k m} \delta^{k m} U_{i} \\
& +\frac{1}{2}\left(\sigma_{6}+\sigma_{7}\right) \overline{\bar{S}}_{k, m 0} \delta^{k m} U_{i}+\frac{1}{2}\left(\sigma_{6}+\sigma_{7}\right) \overline{\bar{S}}_{0,0 i}+\sigma_{8} \overline{\bar{S}}_{0,00} U_{i}=-\frac{1}{2} \bar{J}_{i}^{(S M)} .
\end{aligned}
$$

I vacuum, written in components $(t, x, y, z)$ or $(0, \alpha)$, these interconnected equations in the "rest" system coordinates will have this form:

$$
\begin{array}{ll}
i=0 & \bar{\sigma}_{1} \overline{\bar{S}}_{0,00}+\bar{\sigma}_{2} \overline{\bar{S}}_{0, \alpha \beta} \delta^{\alpha \beta}+\bar{\sigma}_{3} \overline{\bar{S}}_{\alpha, \beta 0} \delta^{\alpha \beta}=0 \\
i=\alpha & \bar{\sigma}_{4} \overline{\bar{S}}_{\alpha, 00}+\sigma_{1} \overline{\bar{S}}_{\alpha, \mu \nu} \delta^{\mu v}+\bar{\sigma}_{5} \overline{\bar{S}}_{\mu, \nu \alpha} \delta^{\mu v}+\bar{\sigma}_{3} \overline{\bar{S}}_{0,0 \alpha}=0 \\
\text { where } & \delta^{\alpha \beta}=\operatorname{diag}(-1,-1,-1) ; \quad \bar{\sigma}_{1} \equiv \sigma_{1}+\sigma_{2}+\sigma_{3}+\sigma_{4}+\sigma_{5}+\sigma_{6}+\sigma_{7}+\sigma_{8}
\end{array}
$$

$$
\bar{\sigma}_{2} \equiv \sigma_{1}+\sigma_{5} ; \quad \bar{\sigma}_{3} \equiv \sigma_{2}+\sigma_{3}+\frac{1}{2}\left(\sigma_{6}+\sigma_{7}\right) ; \quad \bar{\sigma}_{4} \equiv \sigma_{1}+\sigma_{5} \text {. }
$$


The equation for $\overline{\mathbf{r}}$ obtained by variation of the Lagrangian $\bar{L}^{(r)}$ (90) by $\bar{r}_{i j}$ leads to the equations:

$$
\begin{aligned}
\frac{\delta \bar{L}^{(r)}}{\delta \bar{r}_{i j}}=0=> & \hat{\sigma}_{1} \bar{r}_{i j, 00}+\frac{\hat{\sigma}_{2}}{2}\left(\bar{r}_{0 i, k l} \bar{U}_{j}+\bar{r}_{0 j, k l} \bar{U}_{i}\right) \delta^{k l}+\hat{\sigma}_{3} \bar{r}_{, k l} \delta^{k l} \delta_{i j} \\
& +\hat{\sigma}_{4} \bar{r}_{00, k l} \delta^{k l} \bar{U}_{i} \bar{U}_{j}+\frac{\hat{\sigma}_{5}}{2}\left(\bar{r}_{0 i, 0} \bar{U}_{j}+\bar{r}_{0 j, 0} \bar{U}_{i}\right) \\
& +\hat{\sigma}_{6} \bar{r}_{00,00} \bar{U}_{i} \bar{U}_{j}+\hat{\sigma}_{7} \bar{r}_{, 00} \delta_{i j}+\frac{\hat{\sigma}_{8}}{2}\left(\bar{r}_{, k l} \delta^{k l} \bar{U}_{i} \bar{U}_{j}+\bar{r}_{00, k l} \delta^{k l} \delta_{i j}\right) \\
& +\frac{\hat{\sigma}_{9}}{2}\left(\bar{r}_{, 00} \bar{U}_{i} \bar{U}_{j}+\bar{r}_{00,00} \delta_{i j}\right) \\
& +\frac{\bar{V}_{i, j}+\bar{V}_{j, i}}{4}-\frac{\alpha_{1}}{2} \bar{V}_{k, l} \delta^{k l} \delta_{i j}-\frac{\alpha_{2}}{4}\left(\bar{V}_{i, 0} \bar{U}_{j}+\bar{V}_{j, 0} \bar{U}_{i}\right) \\
& -\frac{\alpha_{3}}{2} \bar{V}_{k, l} \delta^{k l} \bar{U}_{i} \bar{U}_{j}-\frac{\alpha_{4}}{2} \bar{V}_{0,0} \bar{U}_{i} \bar{U}_{j}-\frac{\alpha_{5}}{2} \bar{V}_{0,0} \delta_{i j}=-\frac{1}{2} \overline{\bar{F}}_{i j}^{(r)} .
\end{aligned}
$$

And the "gage" equation is obtained by variation of $L^{(r)}$ with respect to Lagrange multiplier $V_{i}$.

$$
\frac{\delta L^{(r)}}{\delta V_{i}}=0=>\left[\bar{r}_{i j, k} \delta^{j k}-\left(\alpha_{1} \bar{r}_{, i}+\alpha_{2} \bar{r}_{0 i, 0}+\alpha_{3} \bar{r}_{00, i}+\alpha_{4} \bar{r}_{00,0} U_{i}+\alpha_{5} \bar{r}_{, 0} U_{i}\right)\right]=0
$$

All constants in the above equations (93) and (94) are the linear combinations of the $\lambda_{n s}$ constants associated with the choice of the Lagrangian of the TotalMatter-see expr. (30)—and the $\alpha$-constants of "gage".

Let us emphasize that the equations (93) and (94) are the first order approximation with respect to parameter $N L_{p} / L_{a} \quad\left(L_{p}\right.$-Plank length and $L_{a}-$ atomic length) for the system with $N$ number of particles. The functions $\overline{\mathbf{r}}$ and $\overline{\mathbf{S}}$, which are small addition to Minkowski metric and constant time vector correspondingly, are defined by only number of particles $N$ and by parameters of atomic scale: speed of light “ $c$ ", Plank's constant $\hbar$, and particle mass $m_{p}$ (for proton).

In non-static case the equations of motions (93) for the $\overline{\overline{\mathbf{S}}}$ could be, depending on the value of the $\sigma$-constants, either hyperbolic (when $\hat{\sigma}_{1} \hat{\sigma}_{2}>0$ and $\hat{\sigma}_{4} \sigma_{1}>0$ ) or elliptic (when $\hat{\sigma}_{1} \hat{\sigma}_{2}<0$ and $\hat{\sigma}_{4} \sigma_{1}<0$ ). The hyperbolic equations $\left(\partial_{t} \partial_{t}-\partial_{x} \partial_{x}\right)$, like for Electromagnetic field, allow the field to exist (and propagate) on itself. On the other hand, the field governed he elliptic equations $\left(\partial_{t} \partial_{t}+\partial_{x} \partial_{x}\right)$ is not allowed independent existence of the time-oscillating field in vacuum. In elliptic equations the field will modify itself (thus will have time depending behavior), but it will quickly decay to zero any king of oscillating harmonics that it produces.

It is important to note that the possibility of elliptic equations are only due to the existence of unit vector $\mathbf{U}$ or to existence of Gravitational vector $\mathbf{G}$ (or S).

It is always assumed that all equations of Nature are hyperbolic due to the fact that they are governed by Minkowski metric. That also means that equations for metric (as it is in General Relativity) must be hyperbolic. And thus must allow Gravitational waves (metric waves) similar to Electromagnetic waves [37] [38]. 
However, the fact that the LIGO project that ran for almost 50 years had not be able to detect the Grav-waves, might indicate that they don't exist. In other words, the equations for both Grav-vector $\mathbf{G}$ (or $\mathbf{S}$ ) and metric $\mathbf{r}$ are not hyperbolic, but rather elliptical. If we consider a rotation of the Earth around the Sun (or Sun around the center of Galaxy), then from point of view of hyperbolic equations (even if we consider only General Relativity), the change of the metric (as the Earth moves so does the metric created by it) must produce waves (just like moving charge make Electromagnetic waves), which due to the "hyperbolicity" of equations runs away or "radiate" out taking with it some energy and thus forcing the orbit to decay and in the end for Earth (or Sun) to fall on the center of mass against which it rotates. However, if we assume that the equations for the Earth metric is governed by elliptical equations, we get totally different picture. The law of conservation of Energy-Momentum would state that flux on the surface due to the exponential decay in time is zero (no radiation). That means that the change of Total-Energy of Earth-Sun interaction is constant. And that means no degradation of the Earth orbit. The "radiation" from the star (or in our case from the Earth) is possible, but not by metric radiation", but by emitting some Matter. If we choose our $\lambda$-constants based on the requirements to vanish "unwanted" terms-see expr. (53) and on-there is "50-50" chance that the equations for $\overline{\mathbf{S}}$ or $\overline{\mathbf{r}}$ turns out to be hyperbolically or elliptical. That means that we would need to impose additional requirement on them to be one way or the other. And if we choose it to be elliptical, the question is: should it be both or only one. The simple answer is both. It would seem to be logical that the pair $\overline{\mathbf{S}}$ and $\overline{\mathbf{r}}$ are tide together and thus have similar form of descriptionboth elliptical.

Let us now discuss the fluxes $\mathbf{J}^{(s)}$ and $\overline{\overline{\mathbf{F}}}^{(r)}$ that defines the Grav-vector $\mathbf{S}$ (or $\overline{\overline{\mathbf{S}}}$ ) and the metric $\overline{\mathbf{r}}$ accordingly. As we mentioned before the gravitational vector $\overline{\mathbf{S}}$ is a square-vector and thus has units $1 / \mathrm{cm}^{2}$. Its second derivative and thus the flux $\overline{\mathbf{J}}^{(S)}$ will have units $1 / \mathrm{cm}^{4}$, which is the same as energy-momentum tensor $T_{i j}$ (measured in units $\hbar c$ ). And it seems to be logical for the macroscopic system-with large number of particles to equate the flux $\left(J^{(S)}\right)_{i}$ with $(\mathrm{O} i)$ component of the tensor energy-momentum $T_{i j}$

$$
J_{i}^{(S)}=k_{S} \bar{T}_{i j} \bar{U}^{j}=k_{G} \bar{T}_{i 0} \quad k_{S}=\text { const. }
$$

The constant $K_{G}$ is unit-less constant with the value about $\approx 1$ (by order of magnitude) and of course might depend on the form of matter under consideration (like gas, liquid, etc.) and its parameters (like pressure and temperature). Similarly, we can use this approach (justification) for the treatment of metric flux $\overline{\overline{\mathbf{F}}}^{(r)}$. Per our definition of metric-correction $\overline{\mathbf{r}}-r_{i j}=\delta_{i j}+\bar{r}_{i j} / G^{2}$ the $\overline{\mathbf{r}}$ has the units $1 / \mathrm{cm}^{2}$. Its second derivative $\left(\overline{\mathbf{r}}^{\prime \prime}\right)$ has units $1 / \mathrm{cm}^{4}$, which is the same as Energy-Momentum tensor measured in units $\hbar c$. With this justification we can write:

$$
\overline{\bar{F}}_{i j}^{(r)}=k_{r} \bar{T}_{i j}
$$

which transfer the equation for $\overline{\mathbf{r}}$ into "Einstein form". Of course, only sym- 
bolic sense. The $\overline{\mathbf{r}}^{\prime \prime}$ are quite different from Einstein's $G R\left(R_{i j}-\frac{1}{2} R r_{i j}=T_{i j}\right)$.

We need to point out that because we transformed the description of Gravitational field and metric into Minkowski flat space, we automatically obtain the law of conservations that are the consequences of Lagrangianian description. These laws are of "approximate" nature as the flat Minkowski approximation of the space.

Accepting the expr. (96) and (97) we will acquire 2 more laws of conservations that are the consequence of the of the law of conservation of Energy-Momentum:

$$
\overline{\bar{F}}_{i j k}^{(r)} \delta^{j k}=k_{r} \bar{T}_{i j, k} \delta^{j k}=0 ; \quad \bar{J}_{j, k}^{(S)} \delta^{j k}=k_{S} \bar{T}_{i j, k} \bar{U}^{i} \delta^{j k}=0 .
$$

In case of static (independent of time) spherical symmetry the Equation (94) for the metric $\overline{\mathbf{r}}$, defines as $\left(\mathrm{d}^{2} s=\bar{r}_{i j} \mathrm{~d} x^{i} \mathrm{~d} x^{j}=\bar{r}_{0} \mathrm{~d}^{2} t-\bar{r}_{\rho} \mathrm{d}^{2} \rho-\bar{r}_{\Omega} \rho^{2} \mathrm{~d} \Omega\right)$, in vacuum reduce themselves to only two obvious equations as a function of distance $\rho:$

$$
\begin{aligned}
& \bar{r}_{00, i j} \delta^{i j}=0 \text { or } \frac{\partial^{2} \bar{r}_{00}}{\partial x^{2}}+\frac{\partial^{2} \bar{r}_{00}}{\partial y^{2}}+\frac{\partial^{2} \bar{r}_{00}}{\partial z^{2}}=0=>\bar{r}_{00}=\frac{K_{00}}{\rho} K_{00}-\text { const. } \\
& \bar{r}_{, i j} \delta^{i j}=0\left(\text { where } \bar{r}=\bar{r}_{i j} \delta^{i j}\right) \text { or } \frac{\partial^{2} \bar{r}}{\partial x^{2}}+\frac{\partial^{2} \bar{r}}{\partial y^{2}}+\frac{\partial^{2} \bar{r}}{\partial z^{2}}=0=> \\
& \bar{r}=\frac{K_{r}}{\rho} K_{r} \text { - const. or } \bar{r}_{0}-\bar{r}_{\rho}-2 \bar{r}_{\Omega}=\frac{K_{r}}{\rho}
\end{aligned}
$$

where the constants $K_{0}$ and $K_{r}$ are defined by the tensor $\mathbf{F}^{(r)}$ (i.e. $\left.K_{0}=\int \mathrm{d} \rho \rho^{2} F_{00}^{(r)}\right)$. These are exactly the equations and solutions of Einstein General relativity, where $K_{r}=0$ and $\bar{r}_{\Omega}=0$. The only difference we have is that in our derivation we also have selected a special "preferred" system coordinates defined by the gage Equation (95), which reduces to this relation:

$$
\frac{\mathrm{d} \bar{r}_{\rho}}{\mathrm{d} \rho} \rho+2\left(\bar{r}_{\rho}-\bar{r}_{\Omega}\right)=\alpha_{1} \frac{\mathrm{d}\left(\bar{r}_{\rho}+2 \bar{r}_{\Omega}\right)}{\mathrm{d} \rho} \rho-\alpha_{3} \frac{\mathrm{d} \bar{r}_{0}}{\mathrm{~d} \rho} \rho .
$$

From which follows that $\bar{r}_{\rho}=K_{\rho} / \rho$ and $\bar{r}_{\Omega}=K_{\Omega} / \rho$, which also leads to the linear relation between $K_{0}, K_{\rho}$ and $K_{\Omega}$. And if we want that this "rest" system of coordinates were the conformely-euclidian we need to request that $\bar{r}_{\Omega}=\bar{r}_{\rho}$.

It worth mentioning that the Landau gage $\bar{r}_{i j, k} \delta^{j k}=0$ applied to spherical-symmetrical metric $\bar{r}\left(\mathrm{~d}^{2} s=\bar{r}_{\rho} \mathrm{d}^{2} \rho+\bar{r}_{\Omega} \rho^{2} \mathrm{~d} \Omega\right)$ yields this relation:

$$
\begin{aligned}
& \mathrm{d}^{2} s= {\left[\frac{\bar{r}_{\rho}-\bar{r}_{\Omega}}{\rho^{2}} x^{2}+\bar{r}_{\Omega}\right] \mathrm{d}^{2} x+\left[\frac{\bar{r}_{\rho}-\bar{r}_{\Omega}}{\rho^{2}} y^{2}+r_{\Omega}\right] \mathrm{d}^{2} y+\left[\frac{\bar{r}_{\rho}-\bar{r}_{\Omega}}{\rho^{2}} z^{2}+r_{\Omega}\right] \mathrm{d}^{2} z } \\
&+2\left[\frac{\bar{r}_{\rho}-\bar{r}_{\Omega}}{\rho^{2}} x y\right] \mathrm{d} x \mathrm{~d} y+2\left[\frac{\bar{r}_{\rho}-\bar{r}_{\Omega}}{\rho^{2}} x z\right] \mathrm{d} x \mathrm{~d} z+2\left[\frac{\bar{r}_{\rho}-\bar{r}_{\Omega}}{\rho^{2}} y z\right] \mathrm{d} y \mathrm{~d} z \\
& \bar{r}_{i j, k} \delta^{j k}=0=>\bar{r}_{\Omega}=\bar{r}_{\rho}+\frac{1}{2} \rho \bar{r}_{\rho, \rho}
\end{aligned}
$$

which is neither Schwardchild $\left(\bar{r}_{\Omega}=0\right)$ nor conformely-euclidian $\bar{r}_{\Omega}=\bar{r}_{\rho}$ representation (to be precise the deviation from Minkowski metric). The later of 
course is used in the calculation of two standard GR tests-bending of light and drift of Mercury orbit.

The solution for the grav-vector $\overline{\overline{\mathbf{S}}}$ has also an obvious form:

$$
\begin{aligned}
& \overline{\bar{S}}_{x}=\overline{\bar{S}}_{y}=\overline{\bar{S}}_{z}=0 ; \quad \overline{\bar{S}}_{0, x x}+\overline{\bar{S}}_{0, y y}+\overline{\bar{S}}_{0, z z}=J_{0}^{(S)} \\
& \overline{\bar{S}}_{0}=\frac{K_{S}}{\rho} ; \text { where } \overline{\bar{K}}_{S}=\int_{0}^{\infty} J_{0}^{(S)} \rho^{2} \mathrm{~d} \rho .
\end{aligned}
$$

Combining expr. (99) and (100) we get the final form solution for the "single bar" Grav-vector $\overline{\mathbf{s}}$ :

$$
\bar{S}_{0}=\frac{\bar{K}_{S}}{\rho} ; \text { where } \bar{K}_{S}=\left(\beta_{1}+\beta_{2}\right) \bar{K}_{r}+\overline{\bar{K}}_{S} .
$$

Per our assumption that all particle have gravitational field which is accumulated to a very large magnitude, the constant $\bar{K}_{S}$ must be positive. Or more appropriate it should be the same sign for all baryons. It is a condition on the functions $\mathbf{H}$ that are the core of the "source" flux $\mathbf{J}^{(G)}$, which we will address in Section 9-Mass-Matter. Or it could be viewed as one more condition on unknown constants $\lambda$-s-see expr. (12)—as well as $\mu$-s, expr. (30).

One of the most important subject that we have not addressed yet is the subject of motion of the point-mass in gravitational field. Accordingly Einstein's GR this is governed by the Einstein's "geodesic" postulate which states the pointmass body moves along geodesic line of the curved metric.

It need to be pointed out that this postulate cannot be taken as one of fundamental principles of Physics, but only as an approximation for two reasons. First, the motion of the body should be derived from the Lagrangian of the physical description. In other words, this postulate is not necessary. In second, it by its nature applies to the "point-mass" physical configuration. It is totally looses its meaning when we consider a field description of some physical entity-like quantum mechanical description of the electron or as in our case a field description of the Matter (Tensor-Potential P).

In our case the correct approach is to derive the movement of point-mass from the Lagrangian-expr. (74) —of the Matter, which includes the interaction between Matter and Gravitational fields $(\overline{\mathbf{S}}$ and $\overline{\mathbf{r}})$. And based on the results of this derivations we might have a situation that mass-point depends on both the metric $\overline{\mathbf{r}}$ and the Grav-vector $\overline{\overline{\mathbf{S}}}$ and thus on both the metric $\overline{\mathbf{r}}$ and the Grav-vector $\overline{\mathbf{S}}$. If we assume that Einstein's geodesic postulate is true we must impose an additional requirements on $\lambda$-constant to vanish the dependence of mass-point trajectory on the vector $\overline{\mathbf{S}}$.

In this regard it is worthwhile to point out that described above procedure of "decoupling" the Grav-vector $\overline{\mathbf{S}}$ and the metric $\overline{\mathbf{r}}$-where we "shifted" the Grav-vector $\overline{\mathbf{S}}$ and left metric unchanged per expr. (87)-is not unique. One can consider the "decoupling" of $\overline{\mathbf{S}}$ and $\overline{\mathbf{r}}$ by "shifting" only the metric $\overline{\mathbf{r}}$ :

$$
\bar{r}_{i j}=\overline{\bar{r}}_{i j}+\hat{\beta}_{1} \bar{S}_{0} \bar{U}_{i} \bar{U}_{j}+\hat{\beta}_{2} \bar{S}_{0} \delta_{i j}+\hat{\beta}_{3}\left(\bar{S}_{i} \bar{U}_{j}+\bar{S}_{j} \bar{U}_{i}\right)
$$

which in coordinate $(0-\alpha)$ form has this form: 


$$
\begin{aligned}
& \bar{r}_{00}=\overline{\bar{r}}_{00}+\left(\hat{\beta}_{1}+\hat{\beta}_{2}+2 \hat{\beta}_{3}\right) \bar{S}_{0} \bar{S}_{0} ; \\
& \alpha \neq \beta=>\bar{r}_{\alpha \beta}=\overline{\bar{r}}_{\alpha \beta} ; \alpha=\beta=>\bar{r}_{\alpha \alpha}=\overline{\bar{r}}_{\alpha \alpha}-\hat{\beta}_{2} G_{0} .
\end{aligned}
$$

If in the expression (87) the Grav-vector $\overline{\mathbf{G}}$ is "shifted" while the metric $\overline{\mathbf{r}}$ is unchanged, in the expression above the metric $\mathbf{r}$ is "shifted" by three parameters $\hat{\beta}$ while Grav-vector $\overline{\mathbf{G}}$ is unchanged. This means that if we require that Einstein's geodesic postulate holds, we get different conditions on $\lambda$-constants.

In fact both approaches-"shifting $\overline{\mathbf{G}}$ or "shifting $\overline{\mathbf{r}}$-could be combined in more general "decoupling" approach if we consider transitioning to new variables $\overline{\bar{S}}_{i}$ and $\overline{\bar{r}}_{i j}$ accordingly to the following linear expressions:

$$
\begin{aligned}
& \bar{S}_{i}=\overline{\bar{S}}_{i}+\beta_{1}^{(S)} \overline{\bar{S}}_{0} \bar{U}_{i}+\beta_{1}^{(r)} r_{0 i}+\beta_{2}^{(r)} r_{00} \bar{U}_{i}+\beta_{3}^{(r)} r \bar{U}_{i} \text { and } \\
& \bar{r}_{i j}=\overline{\bar{r}}_{i j}+\beta_{2}^{(S)} \overline{\bar{S}}_{0} \bar{U}_{i} \bar{U}_{j}+\beta_{3}^{(S)} \overline{\bar{S}}_{0} \delta_{i j}+\beta_{4}^{(S)}\left(\overline{\bar{S}}_{i} \bar{U}_{j}+\overline{\bar{S}}_{j} \bar{U}_{i}\right) \\
& +\beta_{4}^{(r)}\left(r_{0 i} \bar{U}_{j}+r_{0 j} \bar{U}_{i}\right)+\beta_{5}^{(r)} r_{00} \bar{U}_{i} \bar{U}_{j}+\beta_{6}^{(r)} r_{00} \delta_{i j}+\beta_{7}^{(r)} r \bar{U}_{i} \bar{U}_{j}+\beta_{8}^{(r)} r \delta_{i j} \\
& \text { where } \overline{\bar{r}}=\overline{\bar{r}}_{i j} \delta^{i j} ; \quad \overline{\bar{S}}_{0}=\overline{\bar{S}}_{i} \bar{U}^{i} ; \overline{\bar{r}}_{0 i}=r_{k i} \bar{U}^{k} ; \overline{\bar{r}}_{00}=\overline{\bar{r}}_{i j} \bar{U}^{i} \bar{U}^{j}
\end{aligned}
$$

with total $12 \beta^{(S)}$-s and $\beta^{(r)}$-s constants. These expressions, of course, must be reversible-that is we should be able to express $\overline{\bar{S}}_{i}$ and $\overline{\bar{r}}_{i j}$ thru the variables $\bar{S}_{i}$ and $\bar{r}_{i j}$. This can always be done as long as the $\beta$-constants satisfy some ("non-equal zero" type) conditions.

Writing the expr. (105) above for the spacial components of the metric tensor $r_{\alpha, \beta} \alpha=(x, y, z) \beta=(x, y, z)$ and $\alpha \neq \beta$ we get $\bar{r}_{\alpha, \beta}=\overline{\bar{r}}_{\alpha, \beta}$.

Writing expr. (105) for the metric components " $0-\alpha$ " and $S_{\alpha}$ we get this system of equations:

$$
\bar{r}_{\alpha 0}=\left(1+\beta_{4}^{(r)}\right) \overline{\bar{r}}_{\alpha 0}+\beta_{4}^{(S)} \overline{\bar{S}}_{\alpha} ; \bar{S}_{\alpha}=\overline{\bar{S}}_{\alpha}+\beta_{1}^{(r)} \overline{\bar{r}}_{\alpha 0}=>1+\beta_{4}^{(r)}-\beta_{1}^{(r)} \beta_{4}^{(S)} \neq 0 .
$$

The second "non-equal zero" condition of reversibility comes from contracting the the first line expr. (105) by $\bar{U}^{i}$ and the second line of expr. (105) by $U^{i} U^{j}$ and separately by $\delta^{i j}$ :

$$
\begin{aligned}
& \bar{S}_{0}=\overline{\bar{S}}_{0}\left[1+\beta_{1}^{(S)}\right]+\overline{\bar{r}}_{00}\left[\beta_{1}^{(r)}+\beta_{2}^{(r)}\right]+\overline{\bar{r}} \beta_{3}^{(r)} \\
& \bar{r}_{00}=\overline{\bar{S}}_{0}\left[\beta_{2}^{(S)}+\beta_{3}^{(S)}+2 \beta_{4}^{(S)}\right]+\overline{\bar{r}}_{00}\left[1+2 \beta_{4}^{(r)}+\beta_{5}^{(r)}+\beta_{6}^{(r)}\right]+\overline{\bar{r}}\left[\beta_{7}^{(r)}+\beta_{8}^{(r)}\right] \\
& \bar{r}=\overline{\bar{S}}_{0}\left[\beta_{2}^{(S)}+4 \beta_{3}^{(S)}+2 \beta_{4}^{(S)}\right]+\overline{\bar{r}}_{00}\left[2 \beta_{4}^{(r)}+\beta_{5}^{(r)}+4 \beta_{6}^{(r)}\right]+\overline{\bar{r}}\left[1+\beta_{7}^{(r)}+4 \beta_{8}^{(r)}\right] .
\end{aligned}
$$

In order to reverse the relations the determinant of it should not be zero$\Delta \neq 0$-where:

$$
\Delta=\left|\begin{array}{ccc}
1+\beta_{1}^{(S)} & \beta_{1}^{(r)}+\beta_{2}^{(r)} & \beta_{3}^{(r)}
\end{array}\right|
$$

If we substitute the expressions (105) and expr. (85) into the expr. (76) we 
again get quadratic with respect to $\overline{\bar{S}}_{i}$ and $\overline{\bar{r}}_{i j}$ Lagrangian. The part that contains quadratic with respect to $\mathbf{S}^{\prime}$ terms will have only four constants $\beta_{i}^{(S)} i=1,2,3,4$. The last part of the Lagrangian that is quadratic with respect to $\mathbf{r}^{\prime}$ will depend on $8 \beta^{(r)}$ constants. And the middle part- $\mathbf{S}^{\prime} \mathbf{r}^{\prime}-$ will include all $12 \beta$-s constants.

If we add to this the $5 \alpha$-constants of the gage (85), we will have total 17 constants that we can choose to vanish $L^{(S r)}$ and to simplify $L^{(S)}$ and $L^{(r)}$ Lagrangians. With 7 constants needed for vanishing $L^{(S r)}$ and 4 possible constant available to simplify $L^{(S)}$ we have 6 constants available for us to simplify $\vec{L}^{(r)}$. We can use these constants to yield the dependence mass-point movement along geodesic lines only.

When written in the form (90) the equations for $\overline{\mathbf{r}}$ are independent of the Grav-vector $\overline{\mathbf{S}}$ and thus have exactly the Einstein's form and do not contain "dark matter", not even a gravitational constant (as it is in linearized Einstein's equations).

There are however the differences between this set of equations for the correction of metric $(\overline{\mathbf{r}})$ and Einstein's one.

a) The Lagrangian for the metric correction $(\overline{\mathbf{r}})$ even though quadratic with respect to $\overline{\mathbf{r}}^{\prime}$ in form has different constants as compared to GR.

b) It most likely will have a second derivative of $\bar{r}_{00}$ by time $\left(\bar{r}_{00,00}\right)$ which it does not have in the GR.

c) It is only true in the "special" Minkowski flat system coordinates, that is defined by the gage (85) and associated with it equations on the functions $(\overline{\mathbf{r}})$.

d) We know that it cannot be used for the "large" systems where its own gravitational field is compared to the outside Grav-field. And any attempts to use these equations for the description of Universe, probably Galaxies or near event horizon should be considered as unjustified.

In our approach of description, we started from the curved space and by a way of linearizion came up to a flat Minkowski space and description. If we knew the procedure how to do it, we could at this step derive the point-mass approximation directly from the form of Lagrangian of the Matter and thus the trajectory of the "point mass" test particle.

We now can make one more step further (sort of step in reverse direction) and ask ourselves a question: could all the description of point-mass particle behavior can be absorbed into 10 functions that would represent the curvature of the space. In other words, can we replace partial derivatives inside the Lagrangian $L^{(M)}$ with a covariant derivatives associated with some metric $r^{\text {eff }}$ and add the Einstein equations to complete the description? Or phrase it differently, can we "hide" $L^{\text {Grav }}$ Lagrangian (including Grav-vector $\overline{\mathbf{G}}$ ) in the some effective metric $r^{\text {eff }}$ and Einstein's equations of GR?

If this program is possible, it would lead us directly to the Berkenstein [39] idea of effective space metric, which is the basis of his $\mathrm{TeVeS}$ theory.

In this case, we being in flat Minkowski space, would be in no way able to distinguish whether such curved space $\left(r_{i j}^{\text {eff }}\right)$ is real-that is equal to $\bar{r}_{i j}-$ or not. 
Such determination could only be possible, if we consider a larger system where linear approximation of a weak gravitation is not applicable or where mass-point approximation is clearly is not the case.

In principal one can ask even a more general question: can not only for point-mass but for any Matter describe by the Lagrangian $L^{(M)}$ of this theory (expr. (1)) be equally replaced by a Einstein Lagrangian density $L^{\sqrt{ }}=R\left(r^{\text {eff }}\right)+\frac{K_{N}}{c^{2}} L^{(M)}\left(r^{\text {eff }}\right) \sqrt{\left|\operatorname{det}\left(\bar{r}_{i j}^{\text {eff }}\right)\right|}$ with some effective curved metric space $\mathbf{r}^{\text {eff }}$. It's quite possible that due to the "linear" description of the gravitation, such replacement is always exist. In such case the Einstein equations are always right for any weak gravitational field, except for the fact the it produces not real $(\overline{\mathbf{r}})$, but "effective" curvature $\left(\overline{\mathbf{r}}^{\text {eff }}\right)$ of the space.

\section{Electromagnetic and Other Vector Fields}

In this section we deal with the Lagrangian of the Matter and particular with the Lagrangians for the three vector fields $\mathbf{B}, \mathbf{D}$ and $\mathbf{E}^{*}$. The Lagrangian of the Matter $\bar{L}^{(M)}$ which as we showed earlier can be written as-see expr. (59):

$$
\bar{L}^{(M)} \approx \sum_{n} \sum_{s=\text { allinv }} \lambda_{n s}^{(M)}\left[\overline{\mathbf{M}}^{2}+\overline{\mathbf{M Q}}^{2}+\overline{\mathbf{Q}}^{4}\right]
$$

where $\overline{\mathbf{Q}}$ is a linear function of the tensor $\overline{\mathbf{H}}$ (or $\overline{\mathbf{N}}$ and $\overline{\mathbf{C}}$ ) and the vectors $\overline{\mathbf{B}}, \overline{\mathbf{D}}$ and $\overline{\mathbf{E}}$.

The tensor $\overline{\mathbf{M}}$ is a tensor of Matter, which is Total-Matter without the Gravitational field-no $\mathbf{S}^{\prime}$ and no curvature tensor $R_{i j k l}$. In the expr. above we consider all variables (tensors) as a "bar" tensors-or tensors on top of Minkowski space. This means that all its derivatives as just partial derivatives and manipulation of indeces is done with Minkowski metric $\delta_{i j}=(1,-1,-1,-1)$.

The identification of the Electromagnetic field is not a straight forward procedure. Potentially any of the three vector fields $\overline{\mathbf{B}}, \overline{\mathbf{E}}$ and $\overline{\mathbf{D}}$ could be chosen as one. The problem here is not only to choose the vector field that leads to Maxwell equations (or to equations the closest to Maxwell's), but also to be sure that the remaining two vectors fields are in some sense unique and not just a repetition of the vector field that we had identified with Electromagnetic one. The major difference between the vector fields comes from the fact that today in physics there is only one (apart from gravitation) long-distance field-the Electromagnetic field. That means that the other 2 vector fields-out of total $3 \overline{\mathbf{B}}$, $\overline{\mathbf{E}}$ and $\overline{\mathbf{D}}-$ must be short-distance. The short-distance here means that the "time-component" of that vector does not have $1 / \rho$ asymptotic at infinity, but rather $1 / \rho^{n}, n \geq 2$.

We begin with defining the expression for Total-Matter tensor $\mathbf{M}^{\mathrm{T}}$ by choosing $\mu$-constants as:

$$
\mu_{1}=\mu_{2}=\mu_{10}=\mu_{11}=\mu_{12}=\mu_{13}=\mu_{14}=\mu_{15}=0 ; \mu_{6}=-\mu_{5} ; \mu_{9}=-\mu_{8} .
$$

The tensor of Total-Matter $\left(\mathbf{M}^{\mathrm{T}}\right)$ as a function of $\Gamma$-or its symmetrical 
$\stackrel{s}{\Gamma} \equiv \stackrel{s}{i}_{j k}^{i}=\stackrel{s}{\Gamma}_{j k}^{i}$ and its anti-symmetrical $\stackrel{s}{\Gamma} \equiv \stackrel{a}{\Gamma}_{j k}^{i}=-\Gamma_{j k}^{i}$ parts-has this expression:

$$
\begin{aligned}
& \left(M^{T}\right)_{j k l}^{i}=\stackrel{s}{\Gamma}_{j l, k}^{i}-\stackrel{s}{\Gamma}_{j k, l}^{i}+\stackrel{s}{\Gamma}_{j l}^{m}-\stackrel{s}{\Gamma}_{m l}^{i} \stackrel{s}{\Gamma}_{j k}^{m} \\
& +\mu_{3}\left(\delta_{k}^{i} \Gamma_{j l \mid s}^{a t s^{s}}-\delta_{l}^{i} \Gamma_{v}^{a t s_{v}^{s}}\right)+\mu_{4} \delta_{j}^{i t \Gamma_{k l \mid s}^{s}} \\
& +\mu_{5}\left[\delta_{k}^{i}\left(\stackrel{a}{\Gamma_{j \mid l}}-\stackrel{a}{\Gamma}{ }_{l \mid j}\right)-\delta_{l}^{i}\left(\stackrel{a}{\Gamma_{j \mid k}}-\stackrel{a}{\Gamma_{k \mid j}}\right)\right]+\mu_{7} \delta_{j}^{i}\left(\stackrel{a}{\Gamma_{k \mid l}}-\stackrel{a}{\Gamma_{l \mid k}}\right)
\end{aligned}
$$



$$
\begin{aligned}
& \text { where } \stackrel{a}{\Gamma} \Gamma_{k}=\stackrel{a^{i}}{\Gamma_{i k}} ; \quad \stackrel{a t t^{i}}{\Gamma_{j k}}=\stackrel{a^{i}}{\Gamma_{j k}}-\frac{1}{3}\left(\delta_{j}^{i} \stackrel{a}{\Gamma}_{k}-\delta_{k}^{i} \stackrel{a}{\Gamma}_{k}\right) ; \quad \stackrel{a t l^{i}}{\Gamma_{i k}}=0
\end{aligned}
$$

and "|" represents a covariant derivative "using" $\stackrel{s}{\Gamma}$. Our next step is to rewrite the expression above in a form where instead of $\Gamma_{j k}^{i}$ we use the tensor Potential $\mathbf{P}^{\mathrm{T}}\left(\Gamma_{j k}^{i} \rightarrow P_{j k}^{\mathrm{Ti}}\right)$ :

$$
\begin{aligned}
& \left(M^{\mathrm{T}}\right)_{j k l}^{i}=R_{j k l}^{i}+\stackrel{s}{P}_{j l i}^{\mathrm{T} i}-\stackrel{s}{P}_{j k ;}^{\mathrm{T} i}+\stackrel{s}{P}_{m k}^{\mathrm{T} i} \stackrel{s}{P}_{j l}^{\mathrm{T} m}-\stackrel{s}{P}_{m l}^{\mathrm{T} i} \stackrel{s}{P}_{j k}^{\mathrm{T} m} \\
& +\mu_{3}\left(\delta_{k}^{i} \stackrel{a t l_{j \mid s}^{\mathrm{T} s}}{P_{j \mid s}}-\delta_{l}^{i} \stackrel{a t}{P}_{j k \mid s}^{\mathrm{T} s}\right)+\mu_{4} \delta_{j}^{i}{\stackrel{a t t^{s}}{P}}_{k l \mid s}
\end{aligned}
$$

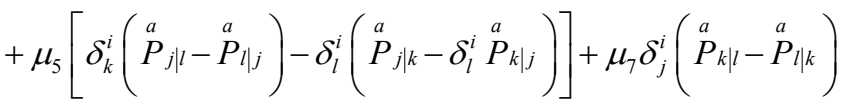

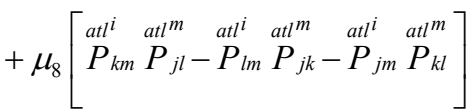



In the expression above we kept the $\mathbf{P}^{\mathrm{T}}$ label on the symmetrical part of the Tensor-Potential so to remind us that it includes the vector $\mathbf{G}$ inside, which is not a part of the anti-symmetrical part of the Tensor-Potential. And in the final step we need to write the tensor of Total-Matter $\mathbf{M}^{\mathrm{T}}$ as a function of sub-fields $H$ (or $\mathbf{N}$ and $\mathbf{C}$ ), $\mathbf{B}, \mathbf{D}$, and $\mathbf{E}^{*}$ (we added a symbol $*$ to indicate that the vector $\mathbf{E}$ always comes as anti-symmetric tensor $\Delta_{i j k l} E^{n}$ ).

The symmetrical part of the Tensor-Potential we has this form:

$$
\begin{aligned}
& \stackrel{s}{P}_{j k}^{T i}=P_{j k}^{(G) i}+P_{j k}^{(B) i}+P_{j k}^{(N) i}+\mu_{0} P^{(K)} \\
& P_{j k}^{(G) i}=r^{i m} G\left[U_{k} r_{m j}+U_{m} r_{j k}+\pi_{1} U_{j} r_{m k}+\pi_{2} U_{j} U_{k} U_{m}\right] ; \\
& P_{j k i}^{(B) i}+\left(\delta_{j}^{i} B_{k}+\delta_{k}^{i} B_{j}+\pi B^{i} r_{j k}\right) \\
& P_{j k}^{(N) i}=r^{i m} N_{m j k} ; \quad N_{i j k}-\text { fully symmetrical and } r^{i j} N_{i j k}=0 \\
& P_{j k}^{(K) i}=r^{i m}\left(C_{j m k}+C_{k m j}\right) \text {-contorsion; } \quad C_{i j k}=-C_{i k j} ; \quad r^{i j} C_{i j k}=0 ; \quad C_{i j k} k^{i j k l}=0
\end{aligned}
$$

and for anti-symmetrical part of of the Tensor-Potential we have:

$$
\stackrel{a}{P} \equiv D_{i} ; \quad \stackrel{a l t}{P}_{j k}^{i} \equiv \stackrel{a}{P}{ }_{j k}^{(C, E) i}=C_{k j}^{i}+\frac{r^{i m} \Delta_{m j k n} E_{s} r^{n s}}{\sqrt{\left|\operatorname{det}\left(r_{p q}\right)\right|}} .
$$


The tensor I that comes with a factor $G^{2}$ comes from quadratic expression for $P^{(G)}$ :

$$
\begin{aligned}
G^{2} \mathbf{I} \equiv & G^{2} I_{i j k l}=\left(P_{i m k}^{(G)} P_{n j l}^{(G)}-P_{i m l}^{(G)} P_{n j k}^{(G)}\right) r^{m n} \\
= & G^{2}\left[\pi_{1}\left(r_{i k} r_{j l}-r_{i l} r_{j k}\right)+\left(1+\pi_{2}\right)\left(r_{i k} U_{j} U_{l}-r_{i l} U_{j} U_{k}\right)\right. \\
& \left.+\pi_{1}\left(\pi_{1}+\pi_{2}\right)\left(U_{i} U_{k} r_{j l}-U_{i} U_{l} r_{j k}\right)\right] .
\end{aligned}
$$

The tensor $\mathbf{Q}$ is linear with respect to any sub-field $\left(\mathbf{N}, \mathbf{C}, \mathbf{B}, \mathbf{D}, \mathbf{E}^{*}\right)$. Thus for example $\mathbf{Q}^{(B)}$ for the field $\mathbf{B}$ is defined by this expression:

$$
\begin{aligned}
G \mathbf{Q}^{(B)} \equiv G & Q_{i j k l}^{(B)}=\left(P_{i m k}^{(G)} P_{m j l}^{(B)}+P_{i m k}^{(B)} P_{m j l}^{(G)}\right)-\left(P_{i m l}^{(G)} P_{m j k}^{(B)}+P_{i m l}^{(B)} P_{m j k}^{(G)}\right) \\
=G[ & \left(U_{m} r_{i k}+U_{k} r_{i m}+\pi_{1} U_{i} r_{m k}+\pi_{2} U_{i} U_{m} U_{k}\right)\left(B^{m} r_{i k}+B_{k} r_{i}^{m}+\pi B_{i} r_{k}^{m}\right) \\
& +\left(B_{m} r_{i k}+B_{k} r_{i m}+\pi B_{i} r_{m k}\right)\left(U_{j} r_{l}^{m}+U_{l} r_{j}^{m}+\pi_{1} U^{m} r_{j l}+\pi_{2} U^{m} U_{j} U_{l}\right) \\
& -\left(U_{m} r_{i k}+U_{l} r_{i m}+\pi_{1} U_{i} r_{m k}+\pi_{2} U_{i} U_{m} U_{l}\right)\left(B^{m} r_{i k}+B_{k} r_{i}^{m}+\pi B_{i} r_{k}^{m}\right) \\
& \left.-\left(B_{m} r_{i k}+B_{k} r_{i m}+\pi B_{i} r_{m k}\right)\left(U_{j} r_{l}^{m}+U_{l} r_{j}^{m}+\pi_{1} U^{m} r_{j l}+\pi_{2} U^{m} U_{j} U_{l}\right)\right] .
\end{aligned}
$$

The above expressions can be somewhat simplified. This could be beneficial (and in fact mandatory) if we would be caring out the exact calculations. But for our purposes what is important is the fact that $\mathbf{Q}^{(B)}$ is a function of sub-field B . Similarly we can calculate the expression for the tensor $\mathbf{Q}^{(N)}$.

However, if we are to write the expression for $\mathbf{Q}^{(C)}$, we must point out that unlike for the sub-fields $\mathbf{N}$ and $\mathbf{B}$ where it is defined by symmetrical part of the Tensor-Potential, the $\mathbf{Q}^{(C)}$ will be defined by both symmetric and antisymmetrical part of the Tensor-Potential. The anti-symmetric dependence comes from term representing covariant ("|") derivatives. The term $\stackrel{a t l}{P}{ }_{j l \mid s}^{\mathrm{Ts}}$ (that come with factor $\mu_{3}\left(\delta_{k}^{i}\right)$ in open form has this expr. $\left({ }^{\star}\right.$ need bar $\left.{ }^{\star}\right)$ :

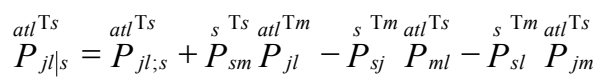

$$
\begin{aligned}
& =\left(C_{j l}^{s}+\frac{1}{\sqrt{\operatorname{det}\left(r_{i j}\right) \mid}} \Delta_{j \ln }^{s} E^{n}\right)_{; s}+\left(P_{s m}^{(G) s}+P_{s m}^{(N) s}+P_{s m}^{(C) s}+P_{s m}^{(B) s}\right)\left(C_{j l}^{m}+\frac{1}{\sqrt{\left|\operatorname{det}\left(r_{i j}\right)\right|}} \Delta_{j \ln }^{m} E^{n}\right) \\
& -\left(P_{s j}^{(G) m}+P_{s j}^{(N) m}+P_{s j}^{(C) m}+P_{s j}^{(B) m}\right)\left(C_{m l}^{s}+\frac{1}{\sqrt{\left|\operatorname{det}\left(r_{i j}\right)\right|}} \Delta_{m \ln }^{s} E^{n}\right) \\
& -\left(P_{s l}^{(G) m}+P_{s l}^{(N) m}+P_{s l}^{(C) m}+P_{s l}^{(B) m}\right)\left(C_{j m}^{s}+\frac{1}{\sqrt{\operatorname{det}\left(r_{i j}\right) \mid}} \Delta_{j m n}^{s} E^{n}\right) .
\end{aligned}
$$

Using (112) and (116) we get this expression for the tensor $\mathbf{Q}^{(C)}$ :

$$
\begin{aligned}
\mathbf{Q}_{i j k l}^{(C)}= & \frac{1}{G} \mu_{0}\left[P_{i k m}^{(G)}\left(C_{j n l}+C_{\ln j}\right) r^{n m}+P_{m j l}^{(G)}\left(C_{n i k}+C_{k i n}\right) r^{n m}\right. \\
& \left.-P_{i l m}^{(G)}\left(C_{j n k}+C_{k n j}\right) r^{n m}+P_{m j k}^{(G)}\left(C_{n i l}+C_{l i n}\right) r^{n m}\right]_{s y m} \\
+ & {\left[\left(P_{s m}^{(G) s} C_{j l}^{m}-P_{s j}^{(G) m} C_{j l}^{m}-P_{s l}^{(G) m} C_{j m}^{s}\right)\right]_{a-s y m} }
\end{aligned}
$$


where we added square brackets with the labels "sym" and "a-sym" to indicate where those terms came from. The exact expression of $\mathbf{Q}^{(C)}$ thru $\mathbf{C}$ is not that important at this point, except for the fact it is linear with respect to $\mathbf{C}$ and it does not depend on value of Grav-vector $\mathbf{G}$, but only on its unit vector $\mathbf{U}$.

For the other two vector fields-D and $\mathbf{E}-$ the $\mathbf{Q}^{(D)}$ and $\mathbf{Q}^{(D)}$ are defined only by anti-symmetrical part of the Tensor-Potential and will depend on the values of $\mu$-constants. It is not difficult to see that for our choice of $\mu$ constants-expr. (112) and (112) $-\mathbf{Q}^{(D)} \equiv 0$.

However for the $\mathbf{Q}^{(E)}$ in general it's not the case. From (112) the expression for $\mathbf{Q}^{(E)}$ has this form:

$$
\begin{aligned}
& Q_{i j k l}^{(E)}=\mu_{3}\left(r_{i k} W_{j l}^{(E)}-r_{i l} W_{j k}^{(E)}\right)+\mu_{4} r_{i j} W_{k l}^{(E)} \text { where } \\
& W_{j l}^{(E)}=\frac{1}{G}\left(P_{s m}^{(G) s} \frac{\Delta_{j \ln }^{m} E^{n}}{\sqrt{\operatorname{det}\left(r_{i j}\right)}}-P_{s j}^{(G) m} \frac{\Delta_{m \ln }^{s} E^{n}}{\sqrt{\operatorname{det}\left(r_{i j}\right) \mid}}-P_{s l}^{(G) m} \frac{\Delta_{j m n}^{s} E^{n}}{\sqrt{\operatorname{det}\left(r_{i j}\right)}}\right) .
\end{aligned}
$$

If we substitute in the expr. above the exact form of $\mathbf{P}^{(G)}$, we get this result for the tensor $W_{j l}^{(E)}$ :

$$
\begin{aligned}
W_{j l}^{(E)} & =\frac{1}{G}\left(P_{s m}^{(G) s} \Delta_{j \mathrm{ln}}^{m} E^{n}-P_{s j}^{(G) m} \Delta_{m \mathrm{ln}}^{s} E^{n}-P_{s l}^{(G) m} \Delta_{j m n}^{s} E^{n}\right) \\
& =E^{n}\left[\left(5+\pi_{1}+\pi_{2}\right) U^{m} \Delta_{m j \mathrm{ln}}-\left(\pi_{1}-1\right) U^{m} r_{l}^{s} \Delta_{s m \mathrm{ln}}-\left(\pi_{1}-1\right) U^{m} r_{l}^{s} \Delta_{s m \mathrm{ln}}\right] \\
& =E^{n}\left(3+3 \pi_{1}+\pi_{2}\right) U^{m} \Delta_{m j \mathrm{ln}} .
\end{aligned}
$$

And if we choose the constants $\pi_{2}=-3\left(1+\pi_{1}\right)$ the $\mathbf{Q}^{(E)} \equiv 0$. This is exactly the reason why we made this choice of $\pi_{2}$-so the $\mathbf{Q}$ contains no vectro field E.

Our next step is to write the tensor of Matter $\mathbf{M}$ as a function of all subfields. Because the tensor of Matter $\mathbf{M}$ is quadratic function with respect to the Tensor-Potential $\mathbf{P}$ when written thru sub-fields $(\mathbf{H}, \mathbf{B}, \mathbf{D}, \mathbf{E})$ it can be split on two groups: sum of sub-fields' Matters and their interaction (labeled $\mathbf{Y}$ ):

$$
\begin{aligned}
M_{i j k l}= & M_{i j k l}^{(H)}+M_{i j k l}^{(B)}+M_{i j k l}^{(D)}+M_{i j k l}^{(E)} \\
& +Y_{i j k l}^{(H B)}+Y_{i j k l}^{(H D)}+Y_{i j k l}^{(H E)}+Y_{i j k l}^{(B D)}+Y_{i j k l}^{(B E)}+Y_{i j k l}^{(D E)} .
\end{aligned}
$$

First we note that because of our choice of $\mu_{15}=0$, the tensor of Matter for D has no quadratic $\mathbf{D}^{2}$ terms and because $\left(\mu_{5}=-\mu_{6}\right)$ it contains the first derivatives $\left(\mathbf{D}^{\prime}\right)$ only in "Maxwellian" form:

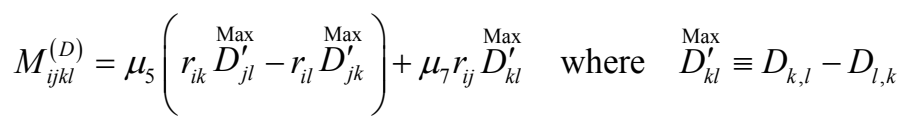

and because $\mu_{11}$ thru $\mu_{14}=0$ all interaction tensors $\left(\mathbf{Y}^{(D B)}, \mathbf{Y}^{(D E)}, \mathbf{Y}^{(D H)}\right)$ are not present.

The situation is somewhat different for the sub-field $\mathbf{E}^{*}$ (we use ${ }^{*}$-symbol to indicate the fact that vector field $\mathbf{E}$ always comes with a fully-anti-symmetrical tensor $\Delta_{i j k l} \frac{1}{\sqrt{\left|\operatorname{det}\left(r_{i j}\right)\right|}}$. 
Because of our choice of the constants $\mu_{1}=\mu_{2}=0$, the tensor of Matter for the vector $\mathbf{E}^{*}\left(\mathbf{M}^{(E)}\right)$ contains the first derivatives only in Maxwellian form:

$$
\begin{aligned}
& M_{i j k l}^{(E)}=\mu_{3}\left(r_{i k} E_{j l}^{* \prime \prime}-r_{i l} E_{j k}^{* \prime}\right)+\mu_{4} r_{i j} E_{k l}^{* \prime} \\
& \text { where } \quad E_{k l}^{* \prime} \equiv\left(E_{p, q}-E_{q, p}\right) r_{s k} r_{t l} \frac{\Delta^{p q s t}}{\sqrt{\operatorname{det}\left(r_{i j}\right)}} .
\end{aligned}
$$

And because per our choice of $\mu_{8}=-\mu_{9}$ and $\mu_{10}=0$ all quadratic terms for $E^{*}\left(\mathbf{E}^{2}\right)$ vanish.

We can now calculate the "interaction" terms $\mathbf{Y}^{(E N)}, \mathbf{Y}^{(E B)}$ and $\mathbf{Y}^{(E C)}$.

The interaction term $\mathbf{Y}^{(E N)}$ comes only from the terms proportional to $\mu_{3}$ and $\mu_{4}$ of the expr. (112):

$$
\begin{aligned}
\mu_{3}\left(\delta_{k}^{i} \stackrel{a t l}{P}_{j l \mid s}^{s}-\delta_{l}^{i} \stackrel{a t l}{P}_{j k \mid s}^{s}\right)+\mu_{4} \delta_{j}^{i} \stackrel{a t}{P}_{k l \mid s} \quad \text { where } \\
\stackrel{a t l^{s}}{P}=\left(C_{j l}^{s}+\Delta_{j \ln }^{s} E^{n}\right)_{, s}+\stackrel{s}{P}_{s m}^{s}\left(C_{j l}^{m}+\Delta_{j \ln }^{m} E^{n}\right) \\
\quad-\stackrel{s}{P}_{s j}^{m}\left(C_{m l}^{s}+\Delta_{m \ln }^{s} E^{n}\right)-\stackrel{s}{P}_{s l}^{m}\left(C_{j m}^{s}+\Delta_{j m n}^{s} E^{n}\right) .
\end{aligned}
$$

Form which follows this expression for $\mathbf{Y}^{(E N)}$

$$
Y_{j l}^{(E N)}=N_{s m}^{s} \Delta_{j \ln }^{m} E^{n}-N_{s j}^{m} \Delta_{m \ln }^{s} E^{n}-N_{s l}^{m} \Delta_{j m n}^{s} E^{n} \equiv 0
$$

due to the fact that $N_{i j k}$ is fully symmetrical and traceless $N_{i j k} r^{i j}=0$.

Unlike for $\mathbf{N}$ the interaction of vector field $\mathbf{E}^{*}$, with the vector $B\left(Y^{(E B)}\right)$ or $C\left(Y^{(E B)}\right)$ do not vanish.

For interaction terms of vector $\mathbf{B}$ and $\mathbf{E}^{*}\left(\mathbf{Y}^{(E B)}\right)$ come from the same expression (112). However now instead of $\stackrel{s}{P}_{j k}^{(G) i}$ we need to use $\stackrel{s}{P}_{j k}^{(B) i}$. Form this follows this expression for $\mathbf{Y}^{(E B)}$ :

$$
\begin{aligned}
& Y_{i j k l}^{(E B)}=\mu_{3}\left(r_{i k} Y_{j l}^{(E B)}-r_{i l} Y_{j k}^{(E B)}\right)+\mu_{4} r_{i j} Y_{k l}^{(E B)} \quad \text { where } \\
& Y_{j l}^{(E B)}=P_{s m}^{(B) s} \Delta_{j \ln }^{m} E^{n}-P_{s j}^{(B) m} \Delta_{m \ln }^{s} E^{n}-P_{s l}^{(B) m} \Delta_{j m n}^{s} E^{n} ; \\
& P_{i j k}^{(B)}=B_{j} r_{i k}+B_{j} r_{i k}+\pi B_{j} r_{i k} \\
& \Rightarrow \quad Y_{j l}^{(E B)}=(3+3 \pi) \Delta_{m j \mathrm{ln}} E^{n} B^{m}
\end{aligned}
$$

and for any choice of $\pi$, except for $\pi=-1$, the interaction terms $\mathbf{Y}^{(E B)}=\mathbf{E}^{*} \mathbf{B}$ do exist. Unfortunately, we cannot use $\pi=-1$ due to the fact that expr, (25) would not be reversible and thus the determination of vectors $\mathbf{B}$ and $\mathbf{G}$ from the Tensor-Potential $\mathbf{P}$ would not be uniquely determined.

For interaction terms for vector $\mathbf{E}^{*}$ and $\mathbf{C}\left(\mathbf{Y}^{(E C)}\right)$ come from two places. First, from the "|" covariant derivatives (112), which would be proportional to the constant $\mu_{0}$ where tensor $\mathbf{C}$ comes thru its con-torsion form. And secondly, from the quadratic terms associated with constants $\mu_{8}$ - see expr. (1) which is due to relation $\mu_{9}=-\mu_{8}$ is fully anti-symmetrical. Using (12) and (124) we have: 


$$
\begin{aligned}
& \hat{C}_{i j k}=\hat{C}_{i k j} \equiv \mu_{0}\left(C_{j i k}+C_{k i j}\right) \Rightarrow> \\
& Y_{i j k l}^{(E C)}=\left\{\mu _ { 3 } \left[r_{i k}\left(\hat{C}_{s m}^{s} \Delta_{j \mathrm{n}}^{m} E^{n}-\hat{C}_{j s}^{m} \Delta_{m \mathrm{ln}}^{s} E^{n}-\hat{C}_{s l}^{m} \Delta_{j m n}^{s} E^{n}\right)\right.\right. \\
&\left.-r_{i l}\left(\hat{C}_{s m}^{s} \Delta_{j k n}^{m} E^{n}-\hat{C}_{j s}^{m} \Delta_{m k n}^{s} E^{n}-\hat{C}_{s k}^{m} \Delta_{j m n}^{s} E^{n}\right)\right] \\
&\left.+\mu_{4} r_{i l}\left(\hat{C}_{s m}^{s} \Delta_{k \mathrm{n}}^{m} E^{n}-\hat{C}_{k s}^{m} \Delta_{m \mid \mathrm{n}}^{s} E^{n}-\hat{C}_{s l}^{m} \Delta_{k m n}^{s} E^{n}\right)\right\} \\
&+\mu_{8}\left[r^{m n} E^{p}\left(C_{i m k} \Delta_{n j l p}+\Delta_{i m k p} C_{n j l}-C_{i m l} \Delta_{n j k p}-\Delta_{i m l p} C_{m j k}-C_{i m j} \Delta_{n k l p}-\Delta_{i m j p} C_{n k l}\right)\right] .
\end{aligned}
$$

The expr. above could be simplified: some terms vanish, some could be combined together. The actual expression of (128) is not that important (at this moment), but what is important here is that the interaction of $\mathbf{E}^{*}$ vector and $\mathbf{C}$ tensor does exist for all parameters $\mu_{3}, \mu_{4}$ and $\mu_{8}$. Combining the results of (124), (127) and (128) we get these expressions for the tensors $\mathbf{M}^{(E)}$ written in symbolic form:

$$
\mathbf{M}^{(E)}=\mathbf{E}^{\text {Max }}+\mathbf{E}^{*} \mathbf{B}+\mathbf{E}^{*} \mathbf{C} .
$$

If we now calculate the expression for the tensor of Matter associated with the vector $\mathbf{B}$ we get all possible terms, which symbolically can be written as:

$$
\mathbf{M}^{(B)}=\mathbf{B}^{\prime}+\mathbf{B}^{2}+\mathbf{B N}+\mathbf{B C}+\mathbf{B E}^{*} ; \quad \mathbf{Q}^{(B)}=\mathbf{U B} \neq 0 .
$$

Important to note that $\mathbf{B}^{\prime}$ in expr. above does not have "Max" lable attache to it, implying that derivatives of $\mathbf{B}$ include all possible terms of $B_{i, j}$-for example, $B_{k ; l} r^{k l} r_{i j}$ or $B_{i ; m} U^{m} U_{j}$. Combining the results of (123), (129) and (130) we get these expressions for the tensors $\mathbf{M}, \mathbf{Q}$ and the Lagrangian of the Matter L:

$$
\begin{aligned}
& \mathbf{M}=\left[\mathbf{H}^{\prime}+\mathbf{H}^{2}\right]_{H}+\left[\mathbf{B}^{\prime}+\mathbf{B}^{2}+\mathbf{B H}\right]_{B}+\frac{\operatorname{Max}}{\overline{\mathbf{D}}^{\prime}}+\left[\begin{array}{l}
\mathrm{Max} \\
\mathbf{E}^{* \prime}
\end{array}+\mathbf{E}^{*} \mathbf{B}+E^{*} \mathbf{C}\right]_{E} ; \\
& \mathbf{Q}=\mathbf{N}+\mathbf{C}+\mathbf{B} \\
& L=\sum_{n} \sum_{s=\text { inv }} \lambda_{n s}\left(\mathbf{M}^{2}+\mathbf{M} \mathbf{Q}^{2}+\mathbf{Q}^{4}\right)
\end{aligned}
$$

where we used square brackets with index " $H$ ", " $B$ " and " $E$ " to indicate the subfields' Lagrangians.

At this point we can transition to the "bar"-variables and to flat Minkowski space $-r_{i j}=>\delta_{i j}$ and $\sqrt{\left|\operatorname{det}\left(r_{i j}\right)\right|} \Rightarrow>1$, so the expr. (131) above takes this form:

$$
\begin{aligned}
& \overline{\mathbf{M}}=\left[\overline{\mathbf{H}}^{\prime}+\overline{\mathbf{H}}^{2}\right]_{H}+\left[\overline{\mathbf{B}}^{\prime}+\overline{\mathbf{B}}^{2}+\overline{\mathbf{B}} \overline{\mathbf{H}}\right]_{B}+\frac{\mathrm{Max}}{\overline{\mathbf{D}}}+\left[\overline{\mathbf{E}}^{* \prime}+\overline{\mathbf{E}}^{*} \mathbf{B}+\overline{\mathbf{E}}^{*} \overline{\mathbf{C}}\right]_{E} ; \\
& \overline{\mathbf{Q}}=\overline{\mathbf{N}}+\overline{\mathbf{C}}+\overline{\mathbf{B}} \\
& \bar{L}=\sum_{n} \sum_{s=\text { inv }} \lambda_{n s} \overline{\mathbf{M}}^{2}+\overline{\mathbf{M}} \overline{\mathbf{Q}}^{2}+\overline{\mathbf{Q}}^{4} .
\end{aligned}
$$

Let us first consider Lagrangian for the vector $\overline{\mathbf{D}}$. It is not difficult to see that in this case the Lagrangian (132) for just the vector $\overline{\mathbf{D}}$ takes this form:

$$
\bar{L}^{(D)}=\left(\frac{\operatorname{Max}}{\overline{\mathbf{D}}^{\prime}}\right)^{2}+\frac{\overline{\mathrm{Max}}^{\prime}}{\mathbf{F}^{(D)}} ; \quad \overline{\mathbf{D}}_{i j}^{\prime}=\bar{D}_{i, j}-\bar{D}_{j, i}
$$


where $\mathbf{F}^{(D)}$ is an anti-symmetric 2-index tensor and is a function of the tensors $\mathbf{H}, \mathbf{B}$ and $\mathbf{E}$. The antisymmetry of $\mathbf{F}^{(D)}$ is due to the antisymmetry of tensor $\frac{\text { Max }}{\mathbf{D}^{\prime}}$. Or reinstating "summation" and the $\lambda$-s:

$$
\bar{L}^{(D)}=\sum_{s=\text { allinv }} \lambda_{s}^{(D)}\left[\left(\overline{\mathbf{D}}^{\prime}\right)^{2}+\frac{\operatorname{Max}}{\overline{\mathbf{D}}^{\prime}} \overline{\mathbf{F}}^{(D)}\right] .
$$

In the index representation $L^{(D)}$ has only 3 invariants and can be written in the following manner:

$$
\begin{aligned}
& L^{D}=\lambda_{1}^{(D)} \frac{\operatorname{Max}}{\bar{D}_{i j}^{\prime}} \frac{\overline{M a x}_{k l}^{\prime}}{\bar{D}_{k l}^{\prime k}} \delta^{j l}+\lambda_{2}^{(D)} \stackrel{\operatorname{Max}}{D_{i j}^{\prime}} \frac{\operatorname{Max}}{D_{k l}^{\prime}} \delta^{i k} \bar{U}^{j} \bar{U}^{l}+\lambda_{3}^{(D)} \frac{\mathrm{Max}}{\bar{D}_{i j}^{\prime}} \delta^{i k} \delta^{j l} \bar{F}_{k l}^{(D)} \\
& \text { or by means of partial integration } \\
& \bar{L}^{D}=\lambda_{1}^{(D)} \frac{\operatorname{Max}}{D_{i j}^{\prime}} \bar{D}_{k l}^{\prime} \delta^{i k} \delta^{j l}+\lambda_{2}^{(D)} \frac{\operatorname{Max}}{D_{i j}^{\prime}} \bar{D}_{k l}^{\prime} \delta \delta^{i k} \bar{U}^{j} \bar{U}^{l}+\lambda_{3}^{(D)} \bar{D}_{i} \delta^{i j} \bar{J}_{j}^{(D)}
\end{aligned}
$$

where $\overline{\mathbf{J}}^{(D)}$ is a 1-index (vector) flux of the field $\mathbf{D}$.

The constants $\lambda_{1}^{(D)}$ thru $\lambda_{3}^{(D)}$ are linear combinations of the $\lambda$-constants of expr. (132) and $\mu$-constants of (132). If we impose one more requirement on the these constants such that $\lambda_{2}^{(D)}=0$, the Lagrangian for the sub-field $\overline{\mathbf{D}}$ takes Maxwellian form - and thus could be a basis for equating the sub-field $\overline{\mathbf{D}}$ with electromagnetic field. However, there is a problem. This Maxwellian (in form) Lagrangian has one peculiar property, which has to do with the form of the flux $\overline{\mathbf{J}}^{(D)}$. Because this flux is the "diversion" of the anti-symmetrical tensor $\overline{\mathbf{F}}^{(D)}\left(\bar{J}_{i}^{(D)}=\bar{F}_{j k, l}^{(D)} \delta^{k l}\right)$ it cannot represent the electrically charged particle. That is, it can not have a solution where vector potential $\overline{\mathbf{D}}$ has $\bar{D}_{0} \approx \frac{Q_{e}}{\rho}$ behavior with respect to distance from the source. Indeed, if such solution existed, then the constant $Q_{e}$ should be found as an integral of the source written in spherical coordinates:

$$
Q_{e}=\int_{\text {all space }} \bar{J}_{0}^{D} \mathrm{~d}^{3} x=4 \pi^{2} \int \bar{J}_{0}^{D} \rho^{2} \mathrm{~d} \rho .
$$

But since the flux is has the property 1 this integral is zero as it follows from these calculations.

$$
\begin{aligned}
\frac{Q_{e}}{4 \pi^{2}} & =\int \bar{J}_{0 k, m}^{D} \delta^{k m} \rho^{2} \mathrm{~d} \rho \\
& =\int_{0}^{\infty} \rho^{2} \mathrm{~d} \rho\left[\left(\rho^{2} \bar{J}_{0 \rho}^{D}\right)_{, \rho} \frac{1}{\rho^{2}}\right]=-\int_{0}^{\infty} \mathrm{d}\left[\rho^{2} \bar{J}_{\rho}^{D}\right]=\left.\left(\rho^{2} J_{\rho}\right)\right|_{0} ^{\infty}=0
\end{aligned}
$$

This will be true for any "well" localized behavior of the flux $\overline{\mathbf{J}}^{(D)}$-that is the flux that has asymptotics faster that $1 / \rho^{2}$. In other words, the static solution for $\overline{\mathbf{D}}$ may not have a distance asymptotic $\approx 1 / \rho$, but it can have the asymptotic of higher order-like $\approx 1 / \rho^{n} n \geq 2$, corresponding to a short range interaction similar to a dipole (thus the letter " $D$ " for this vector field).

It needs to be mentioned here that the idea of representing the flux of a charged particle as a diversions of anti-symmetrical tensor had been put froward by Gustav Mie some 100 years ago. W. Pauli in his "Theory of Relativity" [40] 
has analyze it in some detailed and as an end result showed that it has significant problems-albeit for the different reasons.

In vacuum the Lagrangian $\bar{L}^{(D)}$ is identical to Maxwell one and the $D$-field has the same property as electromagnetic field. So in a language of quantum mechanics we can call it $D$-photon.

Everything that we said above about the sub-field $\overline{\mathbf{D}}$ is in fact true even if we remove the requirement $\lambda_{2}^{(D)}=0$. Since Lagrangian (135) (with $\lambda_{2}^{(D)} \neq 0$ ) depends on anti-symmetrical tensor $\frac{M a x}{\mathbf{D}}$ only, the law of conservation of of the flux $\overline{\mathbf{J}}^{(D)}\left(\bar{J}_{i}^{(D) i}=0\right)$ still holds.

Also, we can "eliminate" the whole factor $\left(1=\lambda_{2}^{(D)}\right)$ if we introduce "scaled time" $\tau \equiv t / \sqrt{1+\lambda^{(D)}}$ and a new vector $\hat{\mathbf{D}} \equiv \hat{D}_{i}=\left(\sqrt{1+\lambda^{(D)}} D_{0}, D_{\alpha}\right)$. In these new variables the Lagrangian of the sub-field $\mathbf{D}$ (or now $\hat{\mathbf{D}}$ ) has this Maxwell form:

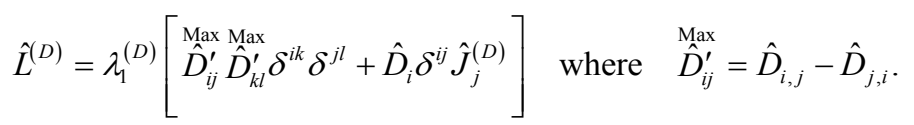

This can be easily seen if we introduce instead 4-dimensional tensor $\stackrel{\text { Max }}{\mathbf{D}} \equiv{\stackrel{\operatorname{Max}}{D_{i j}}} \equiv \bar{D}_{i, j}-D_{j, i}$ two 3-dimensional vectors-“electrical” $\left\{\mathbf{D}^{(\text {elec })} \equiv \mathbf{D}_{\alpha}^{(\text {elec })} \equiv D_{0 \alpha}\right\},(\alpha=x, y, z) \quad$ and “magnetic" $\quad \mathbf{D}^{(\text {magn })} \equiv\left(D_{23}, D_{13}, D_{12}\right)$.

This allows us to rewrite the Lagrangian in this form:

$$
L^{(D)}=\left[1+\lambda^{(D)}\right]\left(\mathbf{D}^{(\mathrm{elec})}\right)^{2}-\left(\mathbf{D}^{(\mathrm{magn})}\right)^{2} \quad \text { where } \quad \lambda^{(D)}=\frac{\lambda^{(D)_{2}}}{\lambda^{(D)_{2}}} .
$$

And if we introduce new vector $\hat{\mathbf{D}} \equiv\left(\sqrt{1+\lambda^{(D)}} D_{0}, D_{\alpha}\right)$ and "scaled time" $\tau \equiv t / \sqrt{1+\lambda^{(D)}} t$, we can rewrite the Lagrangian in Maxwell form:

$$
L^{(D)}=\left(\mathbf{D}^{(\text {elec })}\right)^{2}-\left(\mathbf{D}^{(\text {magn })}\right)^{2} .
$$

It needs to be mentioned that the static solution of equations for sub-field $\overline{\mathbf{D}}$ that come for the Lagrangian (132) (where $\lambda_{2}^{(D)} \neq 0$ ) is identical to the Maxwell static equations. This might make the requirement $\lambda_{2}^{(D)}=0$ not needed.

We now consider Lagrangian for the vector $\mathbf{E}-$ or $\overline{\mathbf{E}}^{*} \equiv \Delta_{i j k m} \bar{E}^{m}$-since it always comes with fully-antisymmetric Levi-Chivita tensor $\Delta^{i j k l}$ (in flat Minkowski space). It is not difficult to see (see expr. (124)) that the Lagrangian for the sub-field $\overline{\mathbf{E}}^{*}$ has this form:

$$
\begin{aligned}
& \begin{array}{l}
\bar{L}^{(E)}=\sum_{n} \sum_{s=\text { allinv }} \lambda_{n s}\left[\left(\overline{\mathbf{M}}^{* *}\right)^{\prime}+\left(\overline{\mathbf{E}}^{*}\right)^{2}(\overline{\mathbf{B}}+\overline{\mathbf{C}})^{2}+\left(\overline{\mathbf{E}}^{*}\right)^{\prime} \overline{\mathbf{F}}^{(E)}\right. \\
\left.+\left(\overline{\mathrm{Max}}^{\prime}\right)\left(\mathbf{E}^{*}\right)(\overline{\mathbf{B}}+\overline{\mathbf{C}})+\overline{\mathbf{E}}^{*}(\overline{\mathbf{B}}+\overline{\mathbf{C}}) \overline{\mathbf{A}}^{(H B)}\right]
\end{array} \\
& \text { where } \quad \overline{\mathbf{M a x}}^{*} \equiv \overline{\mathbf{E}}^{*}{ }_{i j}=\left(\bar{E}_{p, q}-\bar{E}_{q, p}\right) \Delta^{p q m n} \delta_{m_{i}} \delta_{n j} \\
& \text { and } \overline{\mathbf{A}}^{(H B)}=\mathbf{H}^{\prime}+\mathbf{B}^{\prime}+\mathbf{H}^{2}+\mathbf{B}^{2}+\mathbf{H B} .
\end{aligned}
$$

The square of fully anti-symmetric tensor $\Delta_{i j k l}$ (in flat Minkowski space) can be expressed thru Minkowski metric $\delta_{m n}$ and thus in terms with "double star", 
the ** could be dropped. So the final expression for the Lagrangian of the sub-field $\mathbf{E}$ has this form:

$$
\bar{L}^{(E)}=\sum_{n} \sum_{s=a l l i n v} \lambda_{s}^{(E)}\left[\left(\overline{\overline{\mathbf{M a x}}^{\prime}}\right)^{2}+\underline{\mathbf{E}^{2}(\overline{\mathbf{B}}+\overline{\mathbf{C}})^{2}}+\underline{\underline{\underline{\mathrm{Max}}}} \overline{\underline{\mathbf{E}}(\overline{\mathbf{B}}+\overline{\mathbf{C}})}+\overline{\mathbf{E}} \overline{\mathbf{J}}^{\left(E^{* *}\right)}+\overline{\mathbf{E}} \overline{\mathbf{J}}^{\left(E^{*} A\right)}\right]
$$

This Lagrangian differs from the Maxwell's one by a) having an extra invariant $\bar{E}_{i j} \frac{\operatorname{Max}}{E_{k l}} \delta^{i k} U^{j} U^{l}$ in the first $\left(\frac{\operatorname{Max}}{\overline{\mathbf{E}}^{\prime}}\right)^{2}$ symbolic term and b) by the presence of the underlined symbolic terms. As in the case of the sub-field $\overline{\mathbf{D}}$ we require that the (although as we will discuss it in few paragraphs later it might not be an absolute must) the $\lambda$-constants (and the constants $\mu_{3}, \mu_{4}$ and $\mu_{8}$ ) must satisfy a condition so that the extra term $E_{i j} E_{k l} \delta^{i k} U^{j} U^{l}$ vanishes. In addition we also must require that the $\lambda$-constants (and still undefined constants $\mu_{3}$, $\left.\mu_{4} \mu_{8}\right)$ must satisfy a condition so that all invariants of double underlined symbolic term $\left(\underline{\underline{\underline{\mathbf{E}^{\prime}}} \overline{\mathbf{E}} \overline{\mathbf{B}}}\right)$, which consist of only three invariants, and $\left(\underline{\underline{\underline{\mathbf{E}^{\prime}}} \overline{\mathbf{E}} \overline{\mathbf{C}}}\right)$, which consist of only $x$ invariants, vanish.

$$
\begin{aligned}
\sum_{n} \sum_{s=\text { inv }} \lambda_{s}^{(E)} \frac{\operatorname{Max}}{\overline{\mathbf{E}}^{\prime}} \overline{\mathbf{E}} \overline{\mathbf{B}} \equiv & \lambda_{1}^{\left(E^{\prime} E B\right)} \frac{\mathrm{Max}}{\bar{E}_{i j}^{\prime}} \bar{E}^{i} \bar{B}^{j}+\lambda_{2}^{\left(E^{\prime} E B\right)} \frac{\mathrm{Max}}{\bar{E}_{m j}^{\prime}} \bar{U}^{m} \bar{E}^{j}\left(\bar{B}_{n} \bar{U}^{n}\right) \\
& +\lambda_{3}^{\left(E^{\prime} E B\right)} \frac{\mathrm{Max}}{\bar{E}_{m j}^{\prime}} \bar{U}^{m} \bar{B}^{j}\left(\bar{E}_{n} \bar{U}^{n}\right) \\
\sum_{n} \sum_{s=\text { inv }} \lambda_{s}^{(E)} \frac{\operatorname{Max}}{\mathbf{E}^{\prime}} \overline{\mathbf{E}} \overline{\mathbf{C}} \equiv & \lambda_{1}^{\left(E^{\prime} E C\right)} \frac{\mathrm{Max}^{\prime}}{\bar{E}_{i j}^{\prime}} \bar{E}^{i} \bar{C}^{j}+\lambda_{2}^{\left(E^{\prime} E B\right)} \overline{\mathrm{Max}}_{m j}^{\prime} \bar{U}^{m} \bar{E}^{j}\left(\bar{B}_{n} \bar{U}^{n}\right) \\
& +\lambda_{3}^{\left(E^{\prime} E B\right)} \frac{\mathrm{Max}^{\prime}}{E_{m j}^{\prime}} \bar{U}^{m} \bar{B}^{j}\left(\bar{E}_{n} \bar{U}^{n}\right)
\end{aligned}
$$

This requirement steams from the necessity to avoid non-physical situation in the equations for the vector $\overline{\mathbf{B}}$. Indeed, if such term exists, then in equations for $\overline{\mathbf{B}}$ (or $\overline{\mathbf{C}}$ ) it will correspond to the "source" that is only function of Electromagnetic field $\mathbf{E}$. And since $\overline{\mathbf{B}}$ (or $\overline{\mathbf{C}}$ ) is localized (has asymptotics faster than $1 / \rho^{2}$ ) it would require that $\overline{\mathbf{E}}$ should be localized as well, which of course is not the case because it contradicts to the long-distance property of Electromagnetic filed. Strictly speaking, the underlined terms don't have to vanish, but they must not contradict to the "short" distance of either $\mathbf{B}$ or $\mathbf{C}$ at least in static solution. But for now we assume double underlined terms must vanished.

In order for the $L^{(E)}$ Lagrangianin-expr. (142)-to have the Maxwell's form and (thus to represent Electromagnetic field), we must demand that the single underlined term vanishes as well, although as we will discuss it in few paragraphs below this might not be an absolute must-and in fact it might better represent the physical reality.

If we drop all the unwanted terms we get this expression for the $L^{(E)}$ :

$$
L^{(E)}=\bar{E}_{i j} \bar{E}_{k l} \delta^{i k} \delta^{j l}+\bar{E}_{i} \delta^{i j} \bar{J}_{j}^{\left(E^{*}\right)} \quad \text { where } \bar{J}_{j}^{(E)}=\bar{F}_{j k, l}^{\left(E^{* *}\right)} \delta^{k l}-\bar{F}_{j k}^{\left(E^{*} A\right)} .
$$


In the expression above we split the Electromagnetic flux on two components: first, $\left(\frac{\overline{\mathbf{E}}^{*}}{\overline{\mathbf{E}}^{*}}\right)^{\prime} \overline{\mathbf{F}}^{(E)}$, that was derived from the term proportional to $\frac{\overline{\mathbf{E}}^{\prime}}{\overline{\mathbf{E}}^{\prime}}$ and the second, that was derived from the term $\overline{\mathbf{E}}^{*} \overline{\mathbf{B}} \overline{\mathbf{A}}^{(H B)}$. There is a significant difference between these two fluxes. The first (see discussion for sub-field $\overline{\mathbf{D}}$ ) always corresponds to the zero total charge while the second can have non-zero total charge.

If we extend this description to a elementary particles such as electron or proton-which in our case are represented by short-distance (or localized) functions $\overline{\mathbf{H}}$ (or $\overline{\mathbf{N}}$ and $\overline{\mathbf{C}}$ ), we must satisfy one more condition, which reflects an experimental fact that the electric charge of a such particles is always \pm 1 . This requirement must be treated as a condition on the constants of integrations for either field $\mathbf{E}$ or mass-matter tensors $\overline{\mathbf{N}}$ and $\overline{\mathbf{C}}$ (or $\overline{\mathbf{H}}$ ).

In order to reduce the Lagrangian (132) to Maxwell's we requested that the $\overline{\mathbf{E}}^{2}(\overline{\mathbf{B}}+\overline{\mathbf{C}})^{2}$ terms vanish. However what we try to show is that because of "Normalization" procedure such requirement is not necessary. Even with the $\mathbf{B}(\overline{\mathbf{B}}+\overline{\mathbf{C}})^{2} \mathbf{E}^{2}$ terms present this Lagrangian could serve as generalized Lagrangian for the Electromagnetic field.

In standard Maxwell equations the total electrical charge $Q_{e}$ of the system is an integral of the flux $\overline{\mathbf{J}}_{0}^{(E)}$ over all 3D-space:

$$
Q_{e}=\int \mathrm{d}^{3} x \bar{J}_{0}^{(E)}={ }^{ \pm} 1 .
$$

Now if we consider Lagrangian (132) with $\overline{\mathbf{E}}^{2}(\overline{\mathbf{B}}+\overline{\mathbf{C}})^{2}$, then due to the fact that $(\overline{\mathbf{C}})$ (and as we will show few paragraphs below $\overline{\mathbf{B}}$ too) is highly localized it might not influence much on description of the sub-field $\overline{\mathbf{E}}$ outside the mass-matter, except for adding some constant to a total charge value:

$$
Q_{e}=Q^{(E C B)}+\int \mathrm{d}^{3} x \bar{J}_{0}^{(E)} \text { where } Q^{(E C B)}=\int \mathrm{d}^{3} x\left[\overline{\mathbf{E}}^{2}(\overline{\mathbf{C}}+\overline{\mathbf{B}})^{2}\right]
$$

which could be scaled down or totally absorbed by the scaling procedure.

To illustrate this point let us consider a toy model that corresponds to a case where Electromagnetic field has only zero (time) component and functions $\mathbf{H}$ (or $\mathbf{C}$ and $\mathbf{N}$ ) depend only on the distance (-spherical symmetry) and could be expressed by a piece-meal functions: that is a constant for the distances smaller than the size of the particle $\rho_{p}-\rho<\rho_{p}$ and zero for the distances $\rho>\rho_{p}$.

In that case the equations for $E_{0}$ (which we will labeled as $\phi$ ) will have this form:

$$
\frac{\mathrm{d}^{2} \phi}{\mathrm{d} \rho^{2}}+\frac{2}{\rho} \frac{\mathrm{d} \phi}{\mathrm{d} \rho}-V_{\varphi} \phi=V_{J} \text { for } 0<\rho<\rho_{p} \text { and } \frac{\mathrm{d}^{2} \phi}{\mathrm{d} \rho^{2}}+\frac{2}{\rho} \frac{\mathrm{d} \phi}{\mathrm{d} \rho}=0 \text { for } \rho>\rho_{p}
$$

where $V_{\phi}$ and $V_{J}$ are constants, which approximate terms $\overline{\mathbf{E}} \overline{\mathbf{C}}^{2}$ and $\overline{\mathbf{J}}^{(E)}$ correspondingly, and $\rho_{p}$ is the size of the particle. The solution for $\phi$-which is regular everywhere inside the "particle"-as a function of distance $\rho$ has this form: 


$$
\begin{aligned}
& \phi=\frac{V_{J}}{V_{\phi}}\left(1+K_{\phi} \frac{\operatorname{sh}\left(\sqrt{V_{\phi}} \rho\right)}{\sqrt{V_{\phi}} \rho}\right) \quad 0<\rho<\rho_{p} \text { and } \phi=\frac{Q_{e}}{\rho} \text { for } \rho>\rho_{p} \\
& \text { thus } Q_{e}=\rho_{p} \frac{V_{J}}{V_{\phi}}\left(1+K_{\phi} \frac{\operatorname{sh}\left(\sqrt{V_{\phi}} \rho_{p}\right)}{\sqrt{V_{\phi}} \rho_{p}}\right)
\end{aligned}
$$

where $K_{\phi}$ is an arbitrary constant of integration. In our "toy model" we must choose the constant $K_{\phi}$ in such a way that the charge of particle $Q_{e}=1$ (or $-1)$. The presence of $\overline{\mathbf{E}}^{2} \overline{\mathbf{B}}^{2}$ term in the Lagrangian for $\overline{\mathbf{E}}$ field will modify the behavior of the $\overline{\mathbf{E}}$ inside the mass-matter (particle) but-due to the Normalization procedure-will not change its asymptotics. In other words, the "Normalization" procedure guarantees that the asymptotic of Electromagnetic potential (in our toy model $-\phi$ ) outside the particle is always $1 / \rho$. And as long as the asymptotics at infinity is still $1 / \rho$, the Maxwell description of the Electromagnetic field of a macro system (in statistical sense) could be recovered by introducing Dirac's $\delta$-function as a single particle's flux.

$\bar{E}_{i j, k}^{*} \delta^{j k}=Q_{e} \delta(\rho)$ for a single particle or $\frac{\bar{M}_{i j, k}^{*}}{*} \delta^{j k}=J_{i}$ for a continuum system

Such $\delta$-function approximation is possible if the "single" particles are far enough from each other (as compared to their size) so we can use the $1 / \rho$ approximation, which might be harder and harder to achieve as the speed of "single" particles increases toward the speed of light.

By maintaining the term $\overline{\mathbf{E}}^{2} \overline{\mathbf{B}}+\overline{\mathbf{C}}^{2}$ in Lagrangian for the vector $\overline{\mathbf{E}}$ we in fact postulating that the standard Maxwell equations for the Electromagnetic field is an approximation of more complex equations (132) derived thru Affine Unification.

The other point that needs to be made is related to the law of charge conservation that is part of the Maxwell equation. However, by itself the law of conservation is not enough, simply because it does not forbid for example for electron to be split on two halves - each with a charge $-1 / 2$ as long as the total charge is still -1 . What makes this impossible is the statement that electron is not splittable. On the other hand if electron is not splittable, the law of conservation of its charge is automatically holds. So we come to the conclusion that the law of charge conservation is more a property of electron localization and it "normalization" procedure than the outcome of equations of motion for the Electromagnetic field.

We can now consider the Lagrangian for the vector $\overline{\mathbf{B}}$. In symbolic writing it has quite different form all due to the fact that $\overline{\mathbf{Q}}$ is a function of the vector $\overline{\mathbf{B}}$ (we will ignore for now vectors $\overline{\mathbf{E}}$ and $\overline{\mathbf{D}}$ ):

$$
\begin{aligned}
& \overline{\mathbf{Q}}=\overline{\mathbf{U}} \overline{\mathbf{B}}+\overline{\mathbf{U}} \overline{\mathbf{H}} ; \quad \overline{\mathbf{M}}=\overline{\mathbf{B}}^{\prime}+\overline{\mathbf{B}}^{2}+\overline{\mathbf{H}}^{\prime}+\overline{\mathbf{H}}^{2} ; \quad \overline{\mathbf{L}}=\overline{\mathbf{M}}^{2}+\overline{\mathbf{M}} \overline{\mathbf{Q}}^{2}+\overline{\mathbf{Q}}^{4}=>
\end{aligned}
$$



where the first 3 terms in expression for $L^{(B)}$ correspond to the vacuum $(\overline{\mathbf{H}}=0)$, the single-underlined terms could be written as a flux $\left(\overline{\mathbf{B}} \mathbf{J}^{(B)}\right)$ and the 
double-underlined terms represent non-linear interaction between vector $\overline{\mathbf{B}}$ and the mass-matter $\overline{\mathbf{H}}$.

We need to be reminded that the invariants are formed not only with Minkowski metric $\delta^{i j}$, but also with a pair of unit vectors $\bar{U}^{i} \bar{U}^{j}$.

In vacuum the Lagrangian $\bar{L}^{(B)}$ represented by the first three terms of (150) consists of 10 invariants -3 for $\overline{\mathbf{B}}^{4}$ invariants and 7 for $\overline{\mathbf{B}}^{\prime} \overline{\mathbf{B}}^{2}$ :

$$
\begin{aligned}
& \overline{\boldsymbol{B}}^{4} \equiv \lambda_{1}^{\text {(Bvac) }} \bar{B}^{4}+\lambda_{2}^{\text {(Bvac) }} \bar{B}^{2}\left(\bar{B}_{i} \bar{U}^{i}\right)^{2}+\lambda_{3}^{\text {(Bvac) }}\left(\bar{B}_{i} \bar{U}^{i}\right)^{4} \\
& \overline{\boldsymbol{B}}^{\prime} \overline{\boldsymbol{B}}^{2} \equiv \lambda_{4}^{(\mathrm{Bvac})} \bar{B}_{i, j} \bar{B}^{i} \bar{B}^{j}+\lambda_{5}^{\text {(Bvac) }} \bar{B}_{i, j} \delta^{i j} \bar{B}^{2}+\lambda_{6}^{\text {(Bvac) }} \bar{B}_{i, j} \delta^{i j}\left(\bar{B}_{m} \bar{U}^{m}\right)^{2}+\lambda_{7}^{\text {(Bvac) }} \bar{B}_{i, j} \bar{U}^{i} \bar{U}^{j} \bar{B}^{2} \\
& +\lambda_{8}^{\text {(Bvac) }} \bar{B}_{i, j} \bar{U}^{i} \bar{U}^{j}\left(\bar{B}_{m} \bar{U}^{m}\right)^{2}+\lambda_{9}^{\text {(Bvac) }} \bar{B}_{i, j} \bar{U}^{i} \bar{B}^{j}\left(\bar{B}^{m} \bar{U}^{m}\right)+\lambda_{10}^{(\text {Bvac })} \bar{B}_{i, j} \bar{U}^{j} \bar{B}^{i}\left(\bar{B}_{m} \bar{U}^{m}\right)
\end{aligned}
$$

where $\bar{B}^{2} \equiv \bar{B}_{i} \bar{B}^{i} \quad$ and $\quad \bar{U}_{i}=(1,0,0,0)$

It is important to point out that in expr. above the terms $\overline{\mathbf{B}}^{\prime}$ are not of "Minkowski type", i.e. anti-symmetric, but of general type-that is includes both anti-symmetrical and symmetrical parts of the tensor $\mathbf{B}^{\prime}=\bar{B}_{i, j}$. This will lead a situation when the Lagrangian for vector $\overline{\mathbf{B}}$ in its square derivatives will contain all 6 possible terms, very much similar to the gravitation vector $\overline{\mathbf{S}}$-see expr. (83). One of the problems here is that unlike for Electromagnetic field (which had been thoroughly studied over last 100 year), we don't have similar knowledge about non-linear fields, like $\overline{\mathbf{B}}$. So we can only limit ourselves to more or less general statements.

Since the Electromagnetic vector $\overline{\mathbf{E}}$ is the only (apart from Gravitational vector) long-distance filed, the sub-field $\overline{\mathbf{B}}$ must be (in vacuum) a short distance field as well.

The vacuum equation of motion $(\delta L / \delta \overline{\mathbf{B}}=0)$ has this form:

$$
\sum_{s} \lambda_{s}\left[\overline{\mathbf{B}}^{\prime \prime}+\overline{\mathbf{B}}^{\prime} \mathbf{B}+\overline{\mathbf{B}}^{3}\right]=0
$$

It is not difficult to see that if we are to look for the long-distance solution ( $B_{0}=K^{(B)} / \rho, K^{(B)}$-constant), we get a quadratic equation for the constant $K^{(B)}$. If we assume that this equation has no roots, we will automatically have the property that vector $\overline{\mathbf{B}}$ cannot be a long-distance vector.

But even if such solution did exit its value $\left(K^{(B)}\right)$ is of order magnitude of the $\lambda$-constants that define the equation (152) - or magnitude of atomic size. The non-linearity prevent the value of vector $\mathbf{B}$ to grow.

If we consider the question of propagation thru vacuum, we will have two possibilities, that reflect the structure of equation (152) for $\mathbf{B}$. The first possibility is when the equation (152) are of hyperbolic type-that is to say that the second derivative by time and the second derivative by spacial coordinates are of apposite sign, $\left(\partial_{t} \partial_{t}-\partial_{x} \partial_{x}\right) \overline{\mathbf{B}}$. In this case the propagation of $\overline{\mathbf{B}}$-vector in vacuum should be possible, because for small amplitudes of $\overline{\mathbf{B}}$ the non-linear terms could be ignored. The non-linearity, however, would be much pronounced for low frequency/high amplitude waves $(\omega / c<\overline{\mathbf{B}}-$ in units $1 / \mathrm{cm})$.

That means that once emitted this B-particle will split on several smaller (in terms of magnitude) but higher frequency (harmonics) particle that would travel 
thru the space as the zero mass particle, very much similar to the regular photons. And because the $\overline{\mathbf{B}}^{\prime}$ contains terms that include the unit vector $\overline{\mathbf{U}}$ (just like in case the Gravitational vector $\overline{\mathbf{S}}$ ) the vacuum speed for such particles might differ from speed of light by some factor around 1 (from 0.1 to 10)-and thus be even larger than the speed of light-but probably not by several factors of magnitude.

In the second possibility that corresponds to the situation when equation (152) are of elliptical type-that is to say that the second derivative by time and the second derivative by spacial coordinates are of the same sign, $\left(\partial_{t} \partial_{t}-\partial_{x} \partial_{x}\right) \overline{\mathbf{B}}$. In this case the propagation is not possible at all.

It seems to be contradictory that in the Minkowski space the equations for the some form of Matter ( $\overline{\mathbf{B}}$ in this case) were not hyperbolic. However, this is quite possible due to the existence of unit vector $\overline{\mathbf{U}}$. In addition to contractions (in forming the invariants) using metric $\delta^{i j}$, there will be terms where contraction is done using a pair of unit vectors $\bar{U}^{i} \bar{U}^{j}$. It is these terms will create some terms $\partial_{i} \partial_{j} \bar{U}^{i} \bar{U}^{j} \mathbf{B}=\partial_{t} \partial_{t} \mathbf{B}$ that will change the sign in front of $\partial_{t} \partial_{t} \mathbf{B}$ from +1 to -1 and thus change the type of equations from hyperbolic to elliptic.

It is interesting to point out that in vacuum there is some interactions between Electromagnetic field $\overline{\mathbf{E}}$ and either field $\overline{\mathbf{D}}$ or $\overline{\mathbf{B}}$. But because in vacuum $\overline{\mathbf{E}}^{\prime}$ comes only in the form of Maxwell (anti-symmetric) tensor $\frac{\text { Max }}{\mathbf{E}^{\prime *}}$, the interaction for the vector $\overline{\mathbf{E}}$ will have a scattering effect (although with some energy loss). However, for the vector $\overline{\mathbf{B}}$, the Electromagnetic field will have an effect of a source, and thus dragging some $\overline{\mathbf{B}}$ along with it.

\section{Mass-Matter}

One the advantages of the theory based on Affine Unification is that it allows us to view the equations for tensor $\mathbf{C}$ and $\mathbf{N}$ (or for complex $\mathbf{H}$ and its complex conjugate) as equations for the atomic matter (mass-matter) - such as electron, proton, etc.- - or at least their classical description or/and approximation. First we need to emphasize that in weak gravitational field (as in our Solar system) these equations are the 3-index equations (with appropriate symmetry). on top of flat Minkowski space with all covariant derivatives replaced by partial derivatives.

Also important to point out that without any specific requirements it would be too unrealistic to expect that the Lagrangian of the Matter does not contain unity vector $\overline{\mathbf{U}}=(1,0,0,0)$.

And because the equations are derived by means of variation of a Lagrangian, they will contained all the "law of conservations", such as conservation of energy-momentum tensor-associated with it. Those "laws" are not totally universal, but only true as approximation in the "weak" gravitational fields. But for an atomic particles, the "correction" associated with non-Minkowski space (or with curvature of space) is about $L_{a} / L_{\text {plank }} \approx 10^{-36}$ for 2 protons.

Besides tensor $\overline{\mathbf{H}}$ these equations will (or may) contain also vector fields $\overline{\mathbf{E}}$, $\overline{\mathbf{D}}$ and $\overline{\mathbf{B}}$, equations for which we have already considered in the previous sec- 
tions. We know for instance that if we consider a charged particle (like electron or proton) the electromagnetic field $\overline{\mathbf{E}}$ must be present. It is not quite clear what role the other fields serve and in which case their presence in the equations could be ignore. Or may be never.

In addition to the fact that these equations-when we consider elementary mass-particles-are written in flat Minkowski space they (the equations) contain no functions associated with the space curvature $(\overline{\mathbf{r}})$ or grav-vector $\overline{\mathbf{G}}$.

They also are highly non-linear-that is the Lagrangian contains terms proportional to third and even forth power of tensor $\overline{\mathbf{H}}$, such as $\overline{\mathbf{H}}^{\prime} \overline{\mathbf{H}}^{2}$ and $\overline{\mathbf{H}}^{4}$. This non-linearity, serves exactly the same purpose as a postulate of quantum mechanics, which states that no two fermion particles can occupy the same space. Or in other words, the super-position of solutions is not a solution. The non-linearity also produces the "localization" of the mass-particle. If the localization is unstable, the mass-particle will eventually disappear passing its energy to other fields, such as vector fields $\overline{\mathbf{E}}, \overline{\mathbf{D}}$ and $\overline{\mathbf{B}}$.

There are two type of "stable" solutions that should be consider. The first one-the obvious one-is a stationary solution, which does not contain time coordinate. The second is the oscillating type of solution, in which the time is present only under some periodic function-like $\sin (\omega t)$ or $\cos (\omega t))$ along with associated with it frequency $\omega$. The second is of course more general solution and does include the first type as a particular case corresponding to $\omega=0$. It is not clear-since we can not simply switch to a Fourier representation-if such solutions (with $\omega \neq 0$ ) do in fact exist for a highly non-linear equations. And if they do, do they produce the discreet states (or quantization) similar to Quantum Mechanics.

It is not the goal of this paper to give a full investigation of the non-linear solutions for the mass-matter tensor $\overline{\mathbf{H}}$. But instead to look at some simple features of such solutions.

First we need to point that the equations of $\overline{\mathbf{H}}$ must be properly symmetrisized accordingly the symmetry of the tensor $\overline{\mathbf{N}}$ or $\overline{\mathbf{C}}$. For example, if we consider in the Lagrangian for Mass-Matter a term:

$$
\bar{L}=\bar{N}_{i j k, l} \bar{N}_{m n p, q} \delta^{i m} \delta^{j n} \delta^{k q} \delta^{l p} ; \quad \delta^{i j}=\operatorname{diag}(1,-1,-1,-1) .
$$

The equations of motion for the tensor $\mathbf{N}$ will have this form:

$$
q^{i j k} \equiv \frac{\delta \bar{L}}{\delta \bar{N}_{i j k}}=\bar{N}_{i j l, m k} \delta^{i m} \delta^{l m}=0 .
$$

But since $\overline{\mathbf{N}}$ has the following linear symmetries $-\bar{N}_{i j k}$ is fully symmetric and $\bar{N}_{i j k} k^{j k}=0$-so should the equations $q_{i j k}$. This lead to the Equation (154) to take this form:

$$
\begin{aligned}
q_{i j k} \equiv & \left(\bar{N}_{i j m, n k}+\bar{N}_{i k m, n j}+\bar{N}_{j k m, n i}\right) \delta^{m n} \\
& -\frac{1}{6} \delta^{m n} \delta^{p q}\left(\bar{N}_{i p m, n q} \delta_{j k}+\bar{N}_{j p m, n q} \delta_{i k}+\bar{N}_{k p m, n q} \delta_{i j}\right)=0 .
\end{aligned}
$$

Likewise if we take a Lagrangian term that contains unit vector $\bar{U}=(1,0,0,0)$ it too must be properly symmetrisized: 


$$
\begin{gathered}
\bar{L}^{H}=\bar{N}_{i j k, l} \bar{N}_{m n p q} \bar{U}^{i} \bar{U}^{m} \delta^{l p} \delta^{k q} \quad q^{i j k} \equiv \operatorname{symm}\left\{\frac{\delta L}{\delta \bar{N}_{i j k}}\right\}=0=> \\
\delta^{n m} \bar{U}^{l}\left[\left(\bar{N}_{i j l, m n} \bar{U}^{l} \bar{U}_{k}+\bar{N}_{i k l, m n} \bar{U}^{l} \bar{U}_{j}+\bar{N}_{k j l, m n} \bar{U}^{l} \bar{U}_{i}\right)\right. \\
\left.-\frac{1}{6} \bar{U}^{l} \bar{U}^{p}\left(\bar{N}_{i l p, m n} r_{j k}+\bar{N}_{j l p, m n} \delta_{i k}+\bar{N}_{k l p, m n} \delta_{i j}\right)\right]=0 \\
\text { or in Minkowski flat coordinates }(0,1,2,3) \equiv(t, x, y, z): \\
\delta^{n m}\left[\left(\bar{N}_{i j 0, m n} \bar{U}_{k}+\bar{N}_{i k 0, m n} \bar{U}_{j}+N_{k j 0, m n} \bar{U}_{i}\right)-\frac{1}{6} \delta^{m n}\left(\bar{N}_{i 00, m n} \delta_{j k}+N_{j 00, m n} \delta_{i k}+\bar{N}_{k 00, m n} \delta_{i j}\right)\right]=0 .
\end{gathered}
$$

\subsection{Localization}

One of the questions that we would like to consider in this section is asymptotic behavior of the $\overline{\mathbf{H}}$ (or $\overline{\mathbf{N}}$ and $\overline{\mathbf{C}}$ ) on large distances $\rho$ or possibility of localized solutions. We must postulate that to be truly localized these solutions (or dependence $\overline{\mathbf{H}}$ vs. distance $\rho$ ) must decay with the distance faster than $1 / \rho$. More realistically like $1 / \rho^{2}$ or even faster. It is not difficult to see that at infinity $(\rho \rightarrow \infty)$ the nonlinear terms in the equations for $\overline{\mathbf{H}}$ will vanish (for example, if $\overline{\mathbf{H}} \approx 1 / \rho^{2}$, then $\overline{\mathbf{H}}^{\prime \prime} \approx 1 / \rho^{4}$, while $\overline{\mathbf{H}}^{\prime} \overline{\mathbf{H}}^{2} \approx 1 / \rho^{7}$ and $\overline{\mathbf{H}}^{4} \approx 1 / \rho^{8}$ ) transferring the equations of motion for the tensor $\mathbf{H}$ to a linear system of equations similar to the equations of QM. This in its turn may produce the "basis" for transitioning (or coupling) this Affine Unification description to linear QM description of elementary particles.

Typically, the equations of motion of second order (second derivatives) - such as for vector potential for electromagnetic field-produce static solutions with an asymptotic $\approx 1 / \rho$. But because the large number of independent functions that describe the tensor $\overline{\mathbf{H}}$, there is a possibility for a static solution with much stronger than $1 / \rho$ behavior at infinity. We can demonstrate it on a particular case, where $\overline{\mathbf{C}}=0$. We don't know for certain if such assumption is physical-it might be that every mass-matter must have both tensors $\mathbf{N}$ and $\overline{\mathbf{C}}$ presentin which case this considerations should be viewed as a toy model. However the conclusion that large number of independent functions in $\mathbf{H}$ can lead to very much localized solutions is still holds.

In case of spherical symmetry $\overline{\mathbf{N}}$ is described by 6 independent functions$\bar{N}_{t}, \bar{N}_{t 2 \rho}, \bar{N}_{t \rho 2}, \bar{N}_{\rho}, N_{t \Omega}, \bar{N}_{\rho \Omega}$-from which the components of the tensor can be easily deduced using the invariant form: $\bar{N}_{i j k} \mathrm{~d} x^{i} \mathrm{~d} x^{j} \mathrm{~d} x^{k}$, which in case spherical symmetry could be written as:

$$
\begin{gathered}
\begin{array}{c}
\bar{N}_{i j k} \mathrm{~d} x^{i} \mathrm{~d} x^{j} \mathrm{~d} x^{k}=\bar{N}_{t t t}(\mathrm{~d} t)^{3}+3 \bar{N}_{t t \rho}(\mathrm{d} t)^{2} \mathrm{~d} \rho+3 \bar{N}_{t \rho \rho} \mathrm{d} t(\mathrm{~d} \rho)^{2}+\bar{N}_{\rho \rho \rho}(\mathrm{d} \rho)^{3} \\
+3 \rho^{2} \bar{N}_{t \Omega} \mathrm{d} t \mathrm{~d}^{2} \Omega+3 \rho^{2} \bar{N}_{\rho \Omega} \mathrm{d} \rho \mathrm{d}^{2} \Omega
\end{array} \\
\text { where } \mathrm{d}^{2} \Omega=\mathrm{d}^{2} \theta+\sin ^{2} \theta \mathrm{d}^{2} \phi .
\end{gathered}
$$

From this immediate follows expressions for the 8 non-zero components of the tensor $\overline{\mathbf{N}}$

$$
\begin{aligned}
& \bar{N}_{t t t} \equiv \bar{N}_{t} ; \quad \bar{N}_{t t \rho} \equiv \bar{N}_{t 2 \rho} ; \quad \bar{N}_{t \rho \rho} \equiv \bar{N}_{t \rho 2} ; \quad \bar{N}_{\rho \rho \rho} \equiv \bar{N}_{\rho} \\
& \bar{N}_{t \theta \theta} V \rho^{2} \bar{N}_{t \Omega} ; \quad \bar{N}_{t \phi \phi} \equiv \rho^{2} \sin ^{2} \theta \bar{N}_{t \Omega} ; \quad \bar{N}_{\rho \theta \theta} \equiv \rho^{2} \bar{N}_{t \Omega} ; \quad \bar{N}_{\rho \phi \phi} \equiv \rho^{2} \sin ^{2} \theta \bar{N}_{t \Omega} \\
& \bar{N}_{t t \theta}=\bar{N}_{t t \phi}=\bar{N}_{t \rho \theta}=\bar{N}_{t \rho \phi}=\bar{N}_{t \theta \phi}=\bar{N}_{\rho \rho \theta}=\bar{N}_{\rho \theta \phi}=\bar{N}_{\theta \theta \theta}=\bar{N}_{\theta \theta \phi}=\bar{N}_{\theta \phi \phi}=\bar{N}_{\phi \theta \phi}=0
\end{aligned}
$$


The condition (in Minkowski flat space) $\bar{N}_{i j k} \delta^{j k}=0$ in curve-linear coordinates lead to these equations:

$$
\begin{aligned}
& \bar{N}_{t \rho \rho}+2 \bar{N}_{t \Omega}-\bar{N}_{t t t}=0 \\
& \bar{N}_{\rho \rho \rho}+2 \bar{N}_{\rho \Omega}-\bar{N}_{t t \rho}=0 \Rightarrow \bar{N}_{t \rho 2}=\bar{N}_{t}-2 \bar{N}_{t \Omega} ; \bar{N}_{t 2 \rho}=\bar{N}_{\rho}+2 \bar{N}_{\rho \Omega}
\end{aligned}
$$

In general the equations $\mathbf{N}^{\prime \prime}=0$ written in spherical coordinates has a form of linear system of 4 equations for 4 functions $-\bar{N}_{t}, \bar{N}_{\rho}, \bar{N}_{t \Omega}, \bar{N}_{\rho \Omega}$ :

$$
\begin{aligned}
& \hat{\alpha}_{1,1} \bar{N}_{t}^{\prime \prime}+\hat{\alpha}_{1,2} \frac{\bar{N}_{t}^{\prime}}{\rho}+\hat{\alpha}_{1,3} \frac{\bar{N}_{t}}{\rho^{2}} \hat{+} \alpha_{1,4} \bar{N}_{\rho}^{\prime \prime}+\hat{\alpha}_{1,5} \frac{\bar{N}_{\rho}^{\prime}}{\rho}+\hat{\alpha}_{1,6} \frac{\bar{N}_{\rho}}{\rho^{2}}+\hat{\alpha}_{1,7} \bar{N}_{t \Omega}^{\prime \prime} \\
& +\hat{\alpha}_{1,8} \frac{\bar{N}_{t \Omega}^{\prime}}{\rho}+\hat{\alpha}_{1,9} \frac{\bar{N}_{t \Omega}}{\rho^{2}}+\hat{\alpha}_{1,10} \bar{N}_{\rho \Omega}^{\prime \prime}+\hat{\alpha}_{1,11} \frac{\bar{N}_{\rho \Omega}^{\prime}}{\rho}+\hat{\alpha}_{1,12} \frac{\bar{N}_{\rho \Omega}}{\rho^{2}}=0 \\
& \ldots \\
& \hat{\alpha}_{4,1} \bar{N}_{t}^{\prime \prime}+\hat{\alpha}_{4,2} \frac{\bar{N}_{t}^{\prime}}{\rho}+\hat{\alpha}_{4,3} \frac{\bar{N}_{t}}{\rho^{2}}+\hat{\alpha}_{4,4} \bar{N}_{v}^{\prime \prime}+\hat{\alpha}_{4,5} \frac{\bar{N}_{\rho}^{\prime}}{\rho}+\hat{\alpha}_{4,6} \frac{\bar{N}_{\rho}}{\rho^{2}}+\hat{\alpha}_{4,7} \bar{N}_{t \Omega}^{\prime \prime} \\
& +\hat{\alpha}_{4,8} \frac{\bar{N}_{t \Omega}^{\prime}}{\rho}+\hat{\alpha}_{4,9} \frac{\bar{N}_{t \Omega}}{\rho^{2}}+\hat{\alpha}_{4,10} \bar{N}_{v}^{\prime \prime}+\hat{\alpha}_{4,11} \frac{\bar{N}_{\rho \Omega}^{\prime}}{\rho}+\hat{\alpha}_{4,12} \frac{\bar{N}_{\rho \Omega}}{\rho^{2}}=0
\end{aligned}
$$

where the symbol (') represent a derivative by distance $\rho-()^{\prime}=\partial() / \partial \rho-$ and $\hat{\alpha}_{p, q}$ are constants with indeces " $p$ " corresponding to the row and " $q$ " corresponding to the the term within the row. The $\hat{\alpha}$-s constants are linear combinations of the constants $\alpha$-s of the expr. (1), which in their turn depend on the constants $\lambda$-s of the Lagrangian 1 .

Asymptotically at large distances the functions $\bar{N}_{t}, \bar{N}_{\rho}, \bar{N}_{t \Omega}$ and $\bar{N}_{\rho \Omega}$ should have behavior $\approx K / \rho^{n}$, where $K$ is a constant. Assuming that all of then have the same " $n$ " (but different $K-K_{t}, K_{\rho}, K_{t \Omega}$ and $K_{\rho \Omega}$ ) and substituting it in the set of above we get a system of 4 linear equations for $K_{t}, K_{\rho}, K_{t \Omega}$, $K_{\rho \Omega}$.

$$
\begin{aligned}
& K_{t}\left[\hat{\alpha}_{1,1} n(n-1)+\hat{\alpha}_{1,2} n+\hat{\alpha}_{1,3}\right]+K_{\rho}\left[\hat{\alpha}_{1,4} n(n-1)+\alpha_{1,5} n+\alpha_{1,6}\right] \\
& +K_{t \Omega}\left[\alpha_{1,7} n(n-1)+\alpha_{1,8} n+\alpha_{1,9}\right]+K_{\rho \Omega}\left[\alpha_{1,10} n(n-1)+\alpha_{1,11} n+\alpha_{1,12}\right]=0 \\
& \ldots \\
& K_{t}\left[\alpha_{4,1} n(n-1)+\alpha_{4,2} n+\alpha_{4,3}\right]+K_{\rho}\left[\hat{\alpha}_{4,4} n(n-1)+\alpha_{4,5} n+\alpha_{4,6}\right] \\
& +K_{t \Omega}\left[\alpha_{4,7} n(n-1)+\alpha_{4,8} n+\alpha_{4,9}\right]+K_{\rho \Omega}\left[\alpha_{4,10} n(n-1)+\alpha_{4,11} n+\alpha_{4,12}\right]=0
\end{aligned}
$$

The system of equations for the constants $K-s$ has a non-zero solution(s) if the determinant of the eq. (162) is zero. This will lead to a 8-power equations for the parameter " $n$ ", which solely depends on the set of constants $\hat{\alpha}-s$ or ultimately on the set of constants $\lambda$-s. Our postulate of "localization" thus requires that the constants $\hat{\alpha}-s$ (and thus the constants $\lambda$-s) were such that the solution of (162) had a root $n \geq 2$. We can look at this problem in slightly different way. We can set $n$ to $2(n=2)$ and view the Equation (162) as requirement for the $\hat{\alpha}-s$ (and thus the constants $\lambda$-s). Similarly, we could choose $n=3$ (or any other value greater that 1 ). Another word, by adding one (or more) condition on parameters $\lambda$-s (or by choosing different forms of Lagrangians) we 
can set the of " $n$ " to any number. The problem here is that we really don't know what number(s) we should pick. So we are left with 2 options: a) set no conditions for " $n$ " and hope that $\lambda$-s themselves will provide a proper value, or b) derive " $n$ " from other mathematical (or physical) considerations and use it to impose additional conditions on parameters $\lambda$-s.

\subsection{Normalization}

There are some requirements that can not be achieved by proper choice of parameters $\lambda$-s and do relay on the constant of integration of each solution for $\overline{\mathbf{H}}$. Among such requirements are the "normalization" requirements.

In the previous section we have discuss the normalization procedure associated with the law of "fixed minimum charge" - such as proton charge to be +1 and never less or more. This means that for any solution $\overline{\mathbf{H}}$ that we identify with a proton, we must get its charge or asymptotic at infinity as a function of distance $\rho$ equal to $1 / 4 \pi \rho$.

The other requirement could be called "Einstein law" which states that energy of particle is $m c^{2}$.

It is not difficult to see that because all equations are derived from unified Affine description, one of the constant of integration is a scale of coordinate. The units of $\overline{\mathbf{H}}$ is $1 / \mathrm{cm}$. If $\overline{\mathbf{H}}$ is a solution, then for any constant $l_{a}$ the $\hat{\mathbf{H}}=\frac{1}{l_{a}} \overline{\mathbf{H}}\left(\frac{x}{l_{a}}\right)$ is a solution too. The tensor energy-momentum derived for function $\overline{\mathbf{H}}$ using corresponding Lagrangian and bring expressed in the units $\hbar c$ has the same units $1 / l_{a}^{4}$. The Einstein law that total energy of a particle (say proton) is $m_{p} c^{2}$ can be written as:

$$
\frac{m_{p} c^{2}}{\hbar c}=\int_{\text {allspace }} \mathrm{d}^{3} x \frac{1}{l_{a}^{4}} T_{00}^{(H)}\left(\frac{x}{l_{a}}\right)=\frac{\int_{\text {allspace }} \mathrm{d}^{3} x T_{00}}{l_{a}}=\frac{K_{p}}{l_{a}} \text { or } l_{a}=\frac{\hbar}{m_{p} c} K_{p} .
$$

Another words, the Einstein law $E=m c^{2}$ is a one particular normalization procedure. From physics point of view this normalization does not explain why electron's mass is 1800 times less than proton's one. Or-using electron characteristic length-it is 1800 times greater than of proton's. It simply assign a proper value. In order to get the factor 1800 we need one more condition that would effectively compared the length between themselves. Perhaps some common asymptotic at infinity. For example if we postulate that at infinity $\overline{\mathbf{H}}$ has the same asymptotic $\approx K / \rho^{2}$, where $K$ is a fixed constant. That will produce a relation between the characteristic length $\left(l_{\text {proton }}\right.$ and $l_{\text {electron }}$, which of course must be 1836, which is additional condition for the $\lambda$-s constants of the right Lagrangian.

\subsection{Particle, Anti-Particle}

It seems logical, considering the frame work of the Eddington Affine derivation, to assume that if a some form of the tensor potential $\overline{\mathbf{P}}$ represents a particle, then $-\overline{\mathbf{P}}$ (minus $\overline{\mathbf{P}}$ ) represents an anti-particle. However, if we consider (in symbolic writing) the Lagrangian for tensor potential $\overline{\mathbf{P}}$ (see expr. (109)) 


$$
\overline{\mathbf{M}}=\overline{\mathbf{P}}^{\prime}+\overline{\mathbf{P}}^{2} \quad \overline{\mathbf{L}}^{(M)}=\sum_{s=\text { inv }} \lambda_{s}\left[\overline{\mathbf{M}}^{2}+\overline{\mathbf{Q}}^{4}+\overline{\mathbf{M}} \overline{\mathbf{Q}}^{2}\right]=\sum_{s=\text { inv }} \lambda_{s}^{(P)}\left[\overline{\mathbf{P}}^{\prime 2}+\overline{\mathbf{P}}^{\prime} \overline{\mathbf{P}}^{2}+\overline{\mathbf{P}}^{4}\right]
$$

we can see that because of the term $\overline{\mathbf{P}}^{\prime} \overline{\mathbf{P}}^{2}$, if $\overline{\mathbf{P}}$ is a solution $-\overline{\mathbf{P}}$ is (in general) not. So the more accurate definition of anti-particle should be: $\overline{\mathbf{P}}$ transfers to $-\overline{\mathbf{P}}$ and the coordinate $x$ transfers to $-x(x=>-x)$. And since $\overline{\mathbf{P}}=0$ is also a solution then annihilation of particles is possible, assuming of course that all other laws of conservations (energy, etc) are preserved.

This immediately could be apply to any mass-matter (particle) $\overline{\mathbf{H}}$ (ignoring for now all the vector field $\overline{\mathbf{D}}, \overline{\mathbf{E}}, \overline{\mathbf{D}}$ ). If particle is described by some solution $\overline{\mathbf{H}}$ then the solution $-\overline{\mathbf{H}}(-x)$ describes anti-particle.

From that point of view both linear photons $(\overline{\mathbf{D}}, \overline{\mathbf{E}})$ that we discussed in Section 8 in vacuum are identical to their anti-photons due to the fact that Lagrangian of these particles is quadratic with respect to their first derivatives.

It is not difficult to see-see expr. (142) that such procedure correspond to the rule: if particle is changed to anti-particle, the electrical charge (current) changes its sign.

However, if we switch from matter (particle) to anti-matter (anti-particle) expression for Energy-Momentum for the Matter $\left(\overline{\mathbf{T}} \equiv \bar{T}_{m}^{n}=\left[\partial \bar{L} / \partial \bar{P}_{j k, n}^{i}\right] \bar{P}_{j k, m}^{i}-\frac{1}{2} \delta_{n}^{m} \bar{L}\right)$ does not change. Similarly, the unit vector $\bar{U}=(1,0,0,0)$ does not change. This is a consequence of the fact that the gravitation vector $\mathbf{S}$ is a square-vector: $S_{i}=G_{i} G$, where $\mathbf{G}$ is deduced directly from the tensor potential $\mathbf{P}^{\mathrm{T}}$. With the change of $\bar{P} \rightarrow-\bar{P}$ and the coordinates $x \rightarrow-x$ the vector $\mathbf{G}$ changes sign, but the square-vector $\mathbf{S}$ remains unchanged.

In conclusion it needs to be pointed out that we take $\overline{\mathbf{H}}$ as a description of a particle (mass-matter) based primarily on its form of 3-index tensor. It is a possibility that this tensor could be "reduced" to simpler forms-such for example as 6 spinors. In that case one might want to associate the simple forms with the "basic particles" and construct the atomic particles from them.

\subsection{Elliptic vs. Hyperbolic Equations}

We have pointed out before (see Section 7 and Section 8) that existence of Unit vector $\overline{\mathbf{U}}$ allows for the equations of Gravitational field $\overline{\mathbf{S}}$ and metric $\overline{\mathbf{r}}$ to be elliptical. This possibility even more important for the mass-matter fields $\overline{\mathbf{H}}$.

The difficulties of establishing gravitational waves (which in fact should have been an easy task taking into account the sophistication of measuring technologies) might be taken as a proof of the fact that Gravitational field and/or metric described by elliptical equations.

However we almost certain that the mass-matter $\overline{\mathbf{H}}$ should be described by elliptical equations. The reason behind it is its localization and its stability. If the equations were elliptic, then any statistical fluctuation or any collision of 2 mass-particles would create deviation in the form of the localized particle which 
would in time degrade to zero. But if the equations are hyperbolic, the deviations are the composition of "running waves" that can live on its own and "run" away (propagate in space) from the particle, taking with it some energy. This, of course would make the particle to degrade and eventually disappear. But some particles-like electron and proton do not disappear. And the only way to assure it is to postulate that the equations that describe mass-field are elliptical.

\subsection{Moving System of Coordinates}

Since equations for mass-matter (proton, electron, etc) are written in tensorial form, the solution in "rest" system coordinate $\left(\overline{\mathbf{H}}^{(v=0)}\right)$, where Minkowski metric is $\operatorname{diag}(1,-1,-1,-1)$ and $\overline{\mathbf{U}}_{i}=(1,0,0,0)$, could be used to obtain a solution $\left(\overline{\mathbf{H}}^{(v)}\right)$ in any moving with a constant speed $\mathbf{v}$ system coordinate.

The equations that describe the mass-matter (electron, proton, etc.) particle $\overline{\mathbf{H}}$ contain unity vector $\bar{U}=(1,0,0,0)$ and thus in general are not Lorentz invariant. That is to say that in "rest" system coordinate, where Minkowski metric is $\operatorname{diag}(1,-1,-1,-1)$ and $\bar{U}_{i}=(1,0,0,0)$, we have one form of Lagrangian (and thus equations), while writing it in moving - say in $\mathrm{z}$ direction (even with constant speed $\mathbf{v}$ ) we get different form simply because the unit vector $\overline{\mathbf{U}}$ in moving coordinate is going to have non-zero " $Z$ " component:

$U_{i}=(\operatorname{coh} \phi, 0,0, \sin \phi) ; \phi \approx v / c \ll 1$.

Another words, the moving system of coordinate are not identical to the "rest" one. The solution for $\overline{\mathbf{H}}$ will be different and will depend on the speed v/c factor both in amplitude and direction sense. This change might be imperceptible for small $\mathbf{v}$, but if we accelerate say ion of helium $\left(\mathrm{He}^{+}\right)$to the speed compared to the speed of light, we might expect that its atomic levels change or it might simply loose the last electron transferring itself into alpha-particle.

Interesting, that beta decay (weak interaction) of large atoms, has the value about $\approx 1 \mathrm{e}-4$ which is comparable to the speed of our Sun moving around the Milky-Way Galaxy $\left(7.6710^{-4} c\right)$. So, we might suggest that Sun's speed (or "non-rest" system of coordinates) is sufficient large (even though it's only $10^{-3}$ of speed of light) to cause large atoms (nuclei) to change its shape enough to cause the decay of those atoms.

This forces us to ask a following question: how does the localized solution for $\mathbf{H}$ look if the "particle" moves with a constant speed $\mathbf{v}$. Let us consider a simple 2 dimensional $(t, x)$ Minkowski flat space toy model, which as we will show has all the elements that are associated with moving mass-matter. We will consider the following Lagrangian of function $\phi$

$$
\begin{aligned}
& L=\phi_{, i} \phi_{, j} \delta^{i j}+\lambda \phi_{, i} \phi_{, j} U_{k} U_{l} \delta^{i k} \delta^{j l}-\mu^{2} \phi \\
& \text { where } U_{i}=\text { const field } U_{i} U_{j} \delta^{i j}=1 ; \quad \delta_{i j}=\operatorname{diag}(1,-1)
\end{aligned}
$$

which leads to the equation

$$
L=\phi_{, i j} \delta^{i j}+\lambda \phi_{, i j} U_{k} U_{l} \delta^{i k} \delta^{j l}+\mu^{2} \phi=0 .
$$

In the "rest" system coordinate where $U_{i}=(1,0)$ the equation has this form: 


$$
(1+\lambda) \phi_{, t}-\phi_{, x x}+\mu^{2} \phi=0
$$

which has static (time independent) solution

$$
\phi=e^{-\mu x} .
$$

If $\lambda=-2$ the Equation (1) take elliptical form, with no "running wave" solutions-the solution in the form $e^{\omega t+k x}$ has real $\omega$

$$
\phi=e^{\omega t+k x} ; \Rightarrow-\omega^{2}+k^{2}+\mu^{2}=0 ; \Rightarrow \omega=-\sqrt{k^{2}+\mu^{2}} .
$$

Our next step is to find the solution for a mass-matter that moves with a constant speed $v$, which of course is a rotation in $t-x$ space that leaves metric unchanged. For that we need to switch to moving system coordinate associated with moving particle and write the Lagrangian and equations and find the static solution in that system coordinate. Since (169) written in tensorial form, switching to moving system coordinate is simple to rename coordinates $t-x$ to $\tau-y$. There is though an exception-the vector $\mathbf{U}$ which in moving system has both components depending on velocity $v$.

$$
\begin{aligned}
& L=\phi_{, i j} \delta^{i j}+\lambda \phi_{, i j} U_{k} U_{l} \delta^{i k} \delta^{j l}+\mu^{2} \phi=0 \quad U_{\tau}=\frac{1}{\sqrt{1-v^{2}}} ; \quad U_{y}=\frac{v}{\sqrt{1-v^{2}}} \\
& \phi_{, \tau \tau}-\phi_{, y y}+\lambda\left[\phi_{, \tau \tau} U_{\tau}^{2}-2 \phi_{, \tau y} U_{\tau} U_{y}+\phi_{, y y} U_{y}^{2}\right]+\mu^{2} \phi=0
\end{aligned}
$$

with the static solution:

$$
\phi=\mathrm{e}^{-\mu_{V} y} ; \quad \mu_{V}=\frac{\mu\left(1-v^{2}\right)}{\sqrt{1-\lambda v^{2}}} .
$$

And with $\lambda=-2$ (our case), we can see that the localization is remained on all velocity's values. However as $v$ approaches to speed of light $(c=1)$ the "particle" get stretched in spacial dimension $\left(\mu_{V} \rightarrow 0\right)$.

In order to see the particle in the rest system coordinate all we need to do is replace $y$ with $x-U_{y} t\left(y=x-U_{y} t=x-\frac{v t}{\sqrt{1-\lambda v^{2}}}\right)$, which would describe a static solution of the particle moving with a constant speed with respect to "rest" system coordinate. If we consider now the possibility of "running waves" solutions-that is the solutions in a form $e^{i \omega \tau+i k y}-$ we get this equation:

$$
\begin{aligned}
& -\omega^{2}+k^{2}+\lambda\left[-\omega^{2} U_{\tau}^{2}+2 \omega k U_{\tau} U_{y}-k^{2} U_{y}^{2}\right]+\mu^{2}=0 \Rightarrow \\
& \omega=\frac{k \lambda U_{\tau} U_{y} \pm \sqrt{\left(k \lambda U_{\tau} U_{y}\right)^{2}+\left[\mu^{2}+k^{2}\left(1-\lambda U_{y}^{2}\right)\right]\left(1+\lambda U_{\tau}^{2}\right)}}{\left(1+\lambda U_{\tau}^{2}\right)} \\
& =\frac{k \lambda U_{\tau} U_{y} \pm \sqrt{\mu^{2}\left(1+\lambda U_{\tau}^{2}\right)+k^{2}(1+\lambda)}}{\left(1+\lambda U_{\tau}^{2}\right)}
\end{aligned}
$$

from which it's easy to see that in our case $(\lambda=-2)$ for all $v$ the the expr. under the square root is negative implying that no "running waves" are possible. In other words, the particle moving with any speed will deform itself, but it will mountain it ellipticity of its equations. Of course this is a toy model and we 
should not put to much into it. It is quite possible that for complex nuclear assemblies (large number of baryons) the deformation due to the high speed could be "too much" for the assembly to stay together and we might expect that at some high velocity such assembly fall apart (decays).

\section{Cosmology}

A cosmological description of the Universe with a vector field as gravitational matter is both difficult and simple. The simplicity comes from its "statistical" uniformity. If we assume that Universe is uniform closed 3D-sphere with oscillating (or static) radius-and this is the only physical and philosophical right assumptions - then the physical point of view, the presence of Gravitational field G allows this interpretation of Universe' behavior. As Universe expands the average gravitational field decreases, the effective value of Newton's gravitational constant increases, which increases gravitational pull of masses. Eventually, this pull will be strong enough to stop the expansion and reverse it to compression of the Universe. At the other end the shrinking of the size of Universe increases the average value of the gravitational field $\mathbf{G}$, effectively decreasing the Newton's gravitational constant and thus decreasing gravitational pull of masses. The mass' kinetic energy (the temperature of Universe) would be large enough to stop contraction of the Universe and reverse it back to expansion, thus leading to the eternal oscillation of the Universe.

The difficulties, on the other hand, lie in its mathematical model. First, since in cosmology we have to assume that gravitational field defined by all the particles of Universe is comparable to the Matter, we cannot use perturbation series (by parameter $G$ ), but must consider the exact Lagrangian. However, we don't know what the exact Lagrangian is until we actually do the analysis that we described in Section 6-Lagrangian. That is we need to find what is the parameter " $n$ " in expr. (12) and then to derive the actual values of all $\lambda$-s constants associated with that Lagrangian. Also we would need to resolve the issue with extra undetermined parameters of some extra invariants. In other words, we would need to show that all $\lambda$-s are uniquely defined. Second, even if we assume that Lagrangian is known, we would be required to perform "averaging" procedure over the functions describing the Matter $\mathbf{M}^{\mathrm{T}}\left(\mathbf{P}^{\prime}, \mathbf{P}^{2}\right)$. The assumptions that matter is uniformly distributed in the Universe, and the assumption that Universe's metric could be approximated with closed Universe 3-dimensional sphere should significantly simplify the mathematical side of the problem. The averaging procedure might require of (or lead to) introduction, in addition to a "density", some statistical functions as "pressure" and "temperature", which in its turn would demand the laws of "state" for those functions, similar to statistical theory of gas. It is not clear what those "laws" are. Could we equate Universe with ideal "gas" or the interaction between Galaxies are strong enough that one must postulate (or derive) more realistic "laws" of non-ideal gas. Third, the Lagrangian $L^{G}$ for the vector field $\mathbf{G}$ would contain not only square of the first derivatives of the Grav-field $\mathbf{G}$, but also higher powers of it. If we assume that 
in expr. (12) $n=4$, then we will have $G^{\prime 4}$. Perhaps it could be shown that these equations yield the results (or reduce to) similar to the ones obtained in the phenomenological theories of Berkenstein's TeVeS [39] and and Milgrom's MOND [41].

The development of Physics - whether we realize it or not-is in large driven by it's mathematical representation. It is based on Einstein General Relativity, that we arrive to such constructions as: Event Horizon, Black Holes, Open and Close Universe, Big Bang, Black Energy, etc. If this Eddington Unification theory with accumulating Gravitational field $\mathbf{G}$ is correct, it is not clear which of these phenomena survive. Or what their mathematical description would be if they still exist. For example, we might learn that "black holes" that don't allow light to escape do exist (which would not be a big surprise to Astronomers), but those "black hole" have no event horizon. In fact, if the "event horizon" did exist, would we see a ring of a bright glow around each black hole, due to the "stacked" light (from outsider point of view) of all the stars that fell into that "hole" over millions and millions year of its history?

There are more questions than answers. For example, we don't know what the value of Gravitational field $\mathbf{G}_{\infty}$ is as compared to the value of the field created by a Galaxy. Can it be that the Gravitational field between Milky Way (our Galaxy) and the Galaxy next to it is much smaller than the Grav-field in our Solar System $\left(G \approx 10^{33} 1 / \mathrm{cm}\right)$ created by our Galaxy. And if that is the case, then may be-even with hyperbolic equations for $\mathbf{G}$ and $\mathbf{r}$-that is the reason why we cannot detect the gravitational waves that should come from other Galaxies. Perhaps they get reflected back just like water waves when they reach the area where level of water is very low. Or perhaps the waves don't come to us because of the non-linearity effects in description of Grav-vector $\mathbf{G}$-both in terms of $\mathbf{G}^{\prime}$ and $\mathbf{G}$. However, if proposed in this paper theory based on accumulative large Grav-field $\mathbf{G}$ reflects the physical reality, it is quite realistic to assume that Einstein's equations (with or without dark matter) can not be used for description of Universe (or Galaxies) and must be viewed only as a linear approximation applicable only for calculating correction of metric and/or Gravitational field (Dark Matter) in a system of week gravitational interactions.

In the end we would like to emphasize that in proposed theory the Gravitation is described only thru atomic parameters-i.e. $\hbar$ as a measure of energy and mass, $c$ being the speed of light and $L_{a}$ being the atomic length-and the number of particle in the Universe. And because of that it is not difficult to see that the scale of the basic parameters of the Universe-the radius of Universe $L_{U}$ (measures in atomic length $L_{U} / L a$ ) and the Time-Scale of the Universe $T_{U}$-are given by a simple relation with respect to the number of particles in the Universe $N$, where both of basic Universe parameters are proportional to a square root of the number of particles: $L_{U} / L_{a}=T_{U} c / L_{a}=\sqrt{N}$, with $N \approx 10^{83}$.

\section{References}

[1] Brans, C. and Dicke, R.H. (1961) Mach's Principal and Relativistic Theory of Gravi- 
tation. Physical Review, 124, 925. https://doi.org/10.1103/PhysRev.124.925

[2] Uzan, J.-P. (2011) Varying Constants, Gravitation and Cosmology. Living Reviews in Relativity, 14, 2. https://doi.org/10.12942//rr-2011-2

[3] Mannheim, P.D. (2007) Conformal Gravity Challenges String Theory. arXiv: gr-qc/0707.2283.

[4] Meierovich, B.E. (2012) To the Theory of the Universe Evolution. arXiv:1201.2562.

[5] Jacobson, T. (2008) Einstein-Aether Gravity: Status Report. gr-cg/arXiv: 0801.1547.

[6] Koivisto, T.S. and Mota, D.F. (2008) Vector Field Models of Inflation and Dark Energy. JCAP 0808; arxiv.org/0805.4229v3.

[7] Armendariz-Picon, C. (2004) Could Dark Energy Be Vector-Like? arXiv:astro-ph/ 0405267.

[8] Kiselev, V.V. (2004) Vector Field as a Quintessence Partner. arXiv:gr-qc/0402095. https://doi.org/10.1088/0264-9381/21/13/014

[9] Jimenez, J.B. and Maroto, A.L. (2008) Viability of Vector-Tensor Theories of Gravity. arXiv: astro-ph /0811.0784.

[10] Boehmer, C.G. and Harko, T. (2007) Dark Energy as a Massive Vector Field. arXiv:gr-qc/0701029. https://doi.org/10.1140/epjc/s10052-007-0210-1

[11] Elliott, J.W., Moore, G.D. and Stoica, H. (2005) Constraining the New Aether: Gravitational Cherenkov Radiation. arXiv:hep-ph/0505211.

[12] Bailey, Q.G. and Kostelecky, A. (2006) Signals for Lorentz Violation in Post-Newtonian Gravity. arXiv:gr-qc/0603030.

[13] Sidharth, B.G. (2010) Matters of Gravity: Dark Matter and the Cosmological Constant. arXiv:1005.0179 [physics.gen-ph].

[14] Clifton, T., Ferreira, P.G., Padilla, A. and Skordis, C. (2011) Modified Gravity and Cosmology. arXiv:1106.2476 [astro-ph.CO].

[15] Bhattacharya, S., Dialektopoulos, K.F., Romano, A.E., Skordis, C. and Tomaras, T.N. (2016) The Maximum Sizes of Large Scale Structures in Alternative Theories of Gravity. arXiv:1611.05055.

[16] Corda, C. (2008) A Non-Geodesic Motion in the $R^{-1}$ Theory of Gravity Tuned with Observations. arXiv:0801.0319. https://doi.org/10.1142/s0217732308026248

[17] Corda, C. (2007) The Production of Matter from Curvature in a Particular Linearized High Order Theory of Gravity and the Longitudinal Response Function of Interferometers. arXiv:astro-ph/0703644.

[18] Hellings, R. and Nordtvedt, K. (1973) Vector-Metric Theory of Gravity. Physical Review D, 7, 3593. https://doi.org/10.1103/PhysRevD.7.3593

[19] Turyshev, S.G. and Toth, V.T. (2010) The Pioneer Anomaly. arXiv:1001.3686. https://doi.org/10.12942/lrr-2010-4

[20] Persic, M., Salucci, P. and Stel, F. (1995) The Universal Rotation Curve of Spiral Galaxies: I. The Dark Matter Connection. arXiv:astro-ph/9506004.

[21] Sofue, Y. and Rubin, V. (2000) Rotation Curves of Spiral Galaxies. arXiv:astro-ph/ 0010594.

[22] Khalil, S. and Munoz, C. (2001) The Enigma of the Dark Matter. arXiv: hep-ph/0110122.

[23] Weyl, H. (1917) Zur Gravitationstheorie. Annalen der Physik, 359, 117. https://doi.org/10.1002/andp.19173591804

[24] Weyl, H. (1919) Eine neue Erweiterung der Relativitätstheorie. Annalen der Physik, 
364, 101-133; Weyl, H. (1919) Annalen der Physik, 59, 101. https://doi.org/10.1002/andp.19193641002

[25] Eddington, A. (1931) Preliminary Note on the Masses of the Electron, the Proton, and the Universe. Proceedings of the Cambridge Philosophical Society, 27, 15. https://doi.org/10.1017/S0305004100009269

[26] Dirac, P.A.M. (1937) The Cosmological Constants. Nature, 139, 323. https://doi.org/10.1038/139323a0

[27] Ray, S., Mukhopadhyay, U. and Ghosh, P.P. (2007) Large Number Hypothesis. arXiv:0705.1836 [gr-qc].

[28] Fock, V.A. (1964) The Theory of Space, Time and Gravitation. Macmillan.

[29] Bogoliubov, N.N. and Shirkov, D.V. (1959) Introduction to the Theory of Quantized Fields. 2nd Printing Edition, Interscience Publishers, 35.

[30] Eddington, A.S. (1923) The Mathematical Theory of Relativity. Cambridge.

[31] Goenner, H.F.M. (2004) On the History of Unified Field Theories. Living Reviews in Relativity, Max Planck Institute for Gravitational Physics.

[32] Poplawski, N.J. (2007) A Unified, Purely Affine Theory of Gravitation and Electromagnetism. arXiv:0705.0351v4 [gr-qc].

[33] Banados, M. and Ferreira, P.G. (2010) Eddington's Theory of Gravity and Its Progeny. arXiv:1006.1769v2 [astro-ph.CO].

[34] Kijowski, J. and Werpachowski, R. (2004) Universality of Affine Formulation in General Relativity Theory. arXiv:gr-qc/0406088v4.

[35] Cervantes-Cota, J.L. and Liebscher, D.-E. (2016) On Constructing Purely Affine Theories with Matter. arXiv:1607.04250 (gr-qc).

[36] Landau, L.D. and Lifshitz, E.M. (1962) The Classical Theory of Fields. AdisonWesley Publ., Reading.

[37] Corda, C. (2009) A Review of the Stochastic Background of Gravitational Waves in f(R) Gravity with WMAP Constrains. arXiv:0901.1193.

[38] Corda, C. (2009) Will Gravitational Waves Confirm Einstein's General Relativity? arXiv:0907.2162.

[39] Bekenstein, J.D. (2004) Relativistic Gravitation Theory for the Modified Newtonian Dynamics Paradigm. Physical Review D, 70, Article ID: 083509. astro-ph/0403694. https://doi.org/10.1103/physrevd.70.083509

[40] Pauli, W. (1958) Theory of Relativity. Pergamon Press, 188.

[41] Bernal, T., Capozziello, S., Hidalgo, J.C. and Mendoza, S. (2011) Recovering MOND from Extended Metric Theories of Gravity. arXiv:1108.5588 [astro-ph.CO]. 
Submit or recommend next manuscript to SCIRP and we will provide best service for you:

Accepting pre-submission inquiries through Email, Facebook, LinkedIn, Twitter, etc. A wide selection of journals (inclusive of 9 subjects, more than 200 journals)

Providing 24-hour high-quality service

User-friendly online submission system

Fair and swift peer-review system

Efficient typesetting and proofreading procedure

Display of the result of downloads and visits, as well as the number of cited articles Maximum dissemination of your research work

Submit your manuscript at: http://papersubmission.scirp.org/

Or contact jhepgc@scirp.org 